DIRECT-CURRENT VERTICAL BLECTRICAL-RESISTIVITY SOUNDINGS

IN THE LOWER PENINSULA OF MICHIGAN

By D. B. Westjohn and P. J. Carter, Jr.

U.S. GEOLOGICAL SURVEY

Open-File Report 89-244

Lansing. Michigan

1989 
DEPARTMENT OF THE INTERIOR

MANURL LUJAN, JR., Secretary

U.S. GEOLOGICAL SURVEY

Dallas L. Peck, Director

For additional information

write to:

District Chief

U.S. Geological Survey 6520 Mercantile Way. Suite 5

Lansing. Michigan 48911
Copies of this report can be purchased from:

U.S. Geological Survey Books and Open-File Reports Box 25425. Federal Center

Denver. Colorado 80225 


\section{CONVERSION TABLE}

For those readers who prefer inch-pound units rather than the metric (International System) units used in this report, the following conversion factors may be used:

Multiply Metric Unit

meter (m)

kilometer $(\mathrm{km})$
By

Length

$$
3.281
$$

0.6214

Area

square kilometer $\left(\mathrm{km}^{2}\right)$
To Obtain Inch-Pound Unit

foot $(f t)$

mile (mi)

Sea level: In this report "sea level" refers to the National Geodetic Vertical Datum of 1929 (NGVD of 1929)--a geodetic datum derived from a general adjustment of the first-order level nets of both the United States and Canada, formerly called "Datum Sea Level of 1929." 


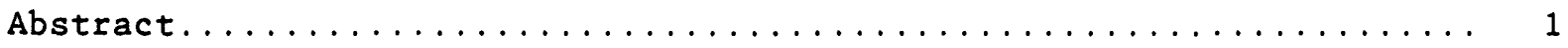

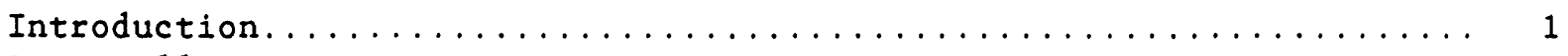

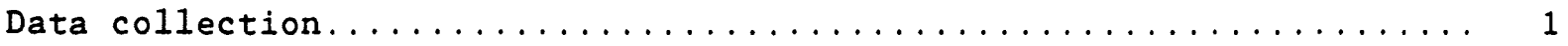

General characteristics of vertical electrical-resistivity

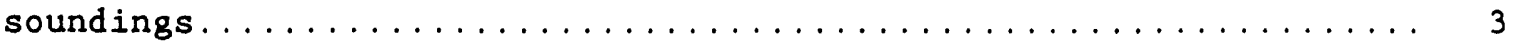

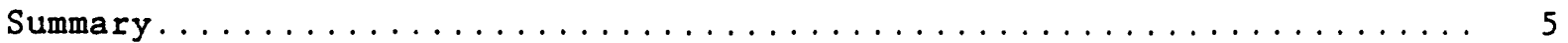

References cited.............................. 6

Appendix: Vertical electrical-resistivity sounding data. shifted data, and calculated electric layers for locations in the Lower Peninsula of Michigan................ 10

\section{ILLUSTRATION}

Figure 1. Map showing location of study area and vertical electrical-resistivity soundings.

\section{TABLE}

Table 1. Summary of observations for vertical electricalresistivity soundings. 


\title{
DIRECT-CURRENT VERTICAL BLECTRICAL-RESISTIVITY SOUNDINGS IN THE LOWER PENINSULA OF MICHIGAN
}

By D. B. Westjohn and P. J. Carter, Jr.

\begin{abstract}
Ninety-three direct-current vertical electrical-resistivity soundings were conducted in the Lower Peninsula of Michigan from June through October 1987. These soundings were made to assist in mapping the depth to brine in areas where borehole resistivity logs and water-quality data are sparse or lacking. The Schlumberger array for placement of current and potential electrodes was used for each sounding. Vertical electrical-resistivity sounding field data, shifted and smoothed sounding data, and electric layers calculated using inverse modeling techniques are presented. Also included is a summary of the near-surface conditions and depths to conductors and resistors for each sounding location.
\end{abstract}

\section{INTRODUCTION}

This report provides geophysical data and inverse model results for 93 direct-current vertical electrical-resistivity soundings conducted in the Lower Peninsula of Michigan from June through October 1987. The Schlumberger electrode array (Zohdy and others, 1974) was used for all soundings. These soundings were made by U.S. Geological Survey personnel as part of the Michigan Basin Regional Aquifer System Analysis (MRASA) project. The geophysical surveys provide data that can be interpreted to indicate the presence of brine in bedrock units below glacial drift, and to investigate areas where freshwater contained in bedrock and glacial aquifers extends to depths that exceed 300 m (meters) (Westjohn, 1989). Vertical electrical-resistivity soundings completed in Michigan in 1986 are summarized by Mandle and Westjohn (1987). Westjohn (1989) provides interpretations for 18 soundings: data for these surveys are included in this report. The purpose of this report is to release the vertical electricalresistivity sounding data and to provide preliminary inverse models for each sounding.

\section{DATA COLLECTION}

The location of each survey site (Figure 1) was selected to provide resistivity data for areas within the MRASA study area where borehole geophysical data are sparse or lacking. Surveys were located in areas that lack obvious cultural interference (powerlines, buried pipelines, and so 


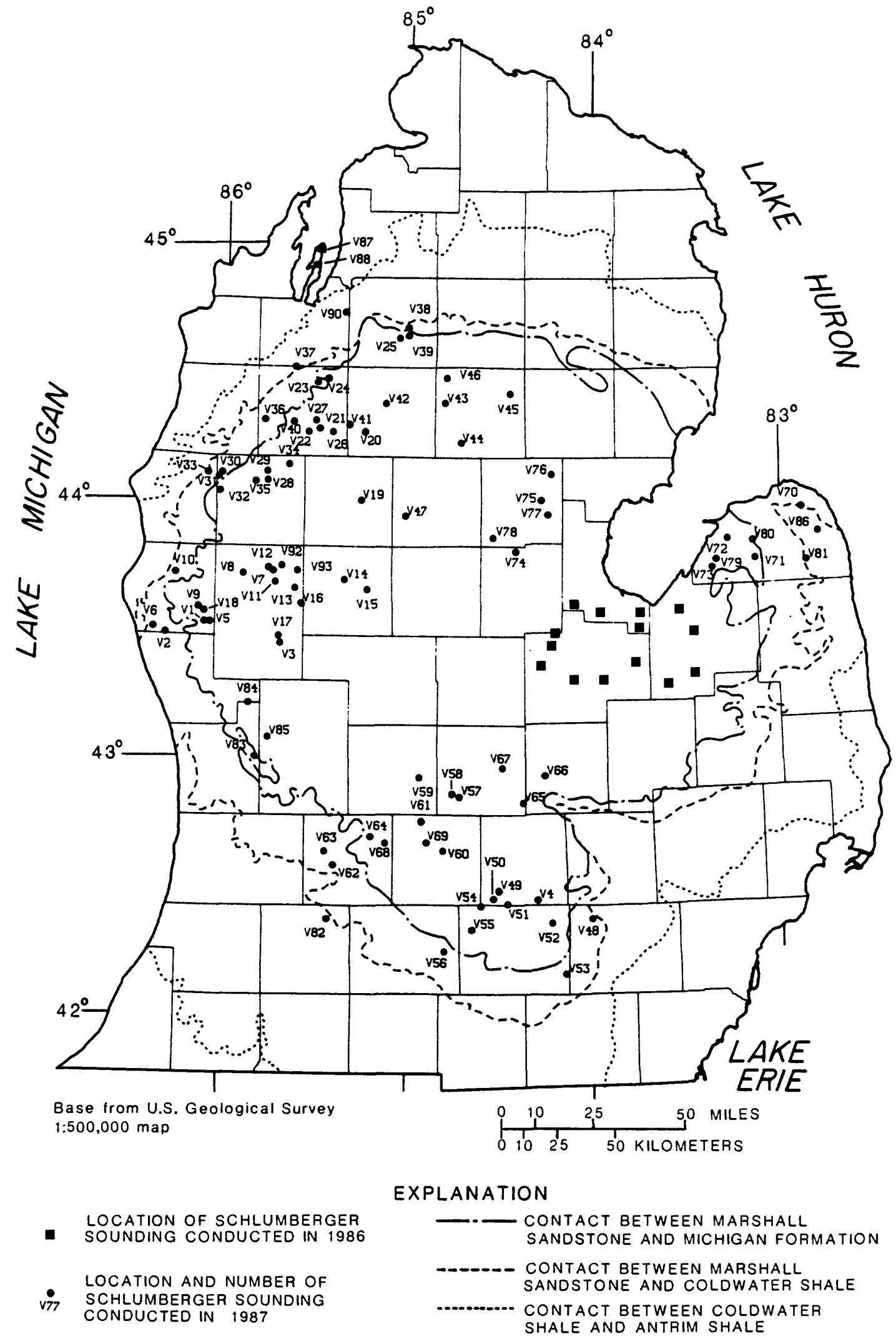

Figure 1.--Location of study area and vertical electricalresistivity soundings. 
forth). Current electrodes for all surveys were expanded to the maximum distance ( 600 to $2.800 \mathrm{~m}$ ) permitted by cultural disturbances and equipment limitations. Equipment used included a Sintrex TSQ-3 square wave transmitter, and RDC-10 receiver ${ }^{1}$. This equipment has a maximum current output of 10 amperes, with a voltage output range of 300 to 1.500 volts. Near-surface geological conditions consisting of well-drained sandy soils constitute the most significant limitation for direct-current electrical-resistivity soundings in the MRASA study area. These soils are very poor conductors, and the amount of current that could be applied to current electrodes under these conditions ( 43 milliamperes to 100 millamperes) was insufficient to produce a clean signal. Electrical potentials recorded by the receiver in cases where applied current ranged from 43 to $100 \mathrm{milliam-}$ peres were often aberant when compared to the overall shape of the field curve. Apparent resistivity data that were not consistent with the general curve shape were not included with field data processed using the shift program of Zohdy (1973).

\section{GENERAL CHARACTERISTICS OF VERTICAL BLECTRICAL-RESISTIVITY SOUNDINGS}

Field data from surveys were processed using programs provided by Zohdy (1973), and Zohdy and Bisdorf (written commun., 1987). Inverse models were generated for each sounding. An inverse program written by Zohdy (1973) provides digital computer interpretation of layer thicknesses and calculates electrical-resistivities that correspond to modeled layers.

Most of the sounding curves for these surveys have forms that are consistent with simple three- or four-layer models. Zohdy and others (1974, p. 25-26) assign single-letter designations for three-layer models. and two-letter designations for four-layer models. The criteria for assigning letter designations to hypothetical three-layer curves are as follows:

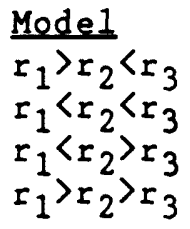

\author{
Curve Type \\ H-type curve \\ A-type curve \\ $\mathrm{K}$-type curve \\ Q-type curve
}

where $r$ is the resistivity of the layer, and the subscript refers to the layer number. For hypothetical four-layer models, the following eight relations exist:

1. Use of trade names in this report is for identification purposes only and does not constitute endorsement by the U.S. Geological Survey. 
Mode1

$$
\begin{aligned}
& r_{1}>r_{2}\left\langler _ { 3 } \left\langle r_{4}\right.\right. \\
& r_{1}>r_{2}\left\langle r_{3}\right\rangle r_{4} \\
& r_{1}\left\langler _ { 2 } \left\langler _ { 3 } \left\langle r_{4}\right.\right.\right. \\
& r_{1}\left\langler _ { 2 } \left\langle r_{3}>r_{4}\right.\right. \\
& r_{1}\left\langle r_{2}>r_{3}\left\langle r_{4}\right.\right. \\
& r_{1}\left\langle r_{2}>r_{3}>r_{4}\right. \\
& r_{1}>r_{2}>r_{3}\left\langle r_{4}\right. \\
& r_{1}>r_{2}>r_{3}>r_{4}
\end{aligned}
$$

Curve Type

$$
\begin{aligned}
& \text { HA-type curve } \\
& \text { HK-type curve } \\
& \text { AA-type curve } \\
& \text { AK-type curve } \\
& \text { KH-type curve } \\
& \text { KQ-type curve } \\
& \text { QH-type curve } \\
& \text { QQ-type curve. }
\end{aligned}
$$

Most of the soundings are consistent with three- or four-layer models, but there are cases where shifted-field curves are more complex (Table 1). Where the curve form cannot be assigned a letter designation consistent with a three- or four-layer model, a numerical value is used in the table to describe the minimum number of layers necessary to explain fluctuations in curve shape. The general shape of each curve is a function of many factors, the most important of which are: (1) surface conditions and depth to water table at the survey site. (2) abrupt deviations in apparent resistivity (cusps, as described by Zohdy and others. 1974. p. 39 ), and (3) substantial vertical or horizontal resistivity contrasts in subsurface layers. Significant contrasts in resistivity for lithologies in the Michigan basin have been reported by Westjohn (1989). Three rock units are assumed to control the bulk of the vertical electrical-resistivity soundings (pronounced conductors and resistors) are (1) anhydrite and dense dolomite in the Michigan Formation with measured resistivities greater than $2.000 \mathrm{ohm}-\mathrm{m}$ (ohm-meters). (2) brine-bearing Marshall Sandstone with measured resistivities less than $5 \mathrm{ohm}-\mathrm{m}$, and (3) the Coldwater Shale with measured resistivities ranging from 9 to $14 \mathrm{ohm}-\mathrm{m}$. The station number, near-surface conditions, curve type, maximum spread between current electrodes $(A B / 2)$. number of apparent resistivity values deleted from the models, and estimated depths to resistors and conductors are listed in Table 1 .

The following items for each sounding are included in the Appendix:

1) A $108-108$ plot of output from the inverse-model program, that shows the digitized data points, curve trace that corresponds to calculated layers, and a trace that shows resistivity and modeled depth for each model layer:

2) A tabulation of raw field data, with location for each survey site listed in the township and range subdivision system:

3) A designation of data points that were excluded before processing field data (denoted by "): these data points are "spikes" or "troughs" (Zohdy and others, 1974) that are interpreted to reflect cultural interferences, current leaks, equipment malfunctions, or abrupt lateral subsurface heterogeneities: and 
4) A tabulation of values for voltage (V), current (I), and calculated apparent resistivity for each current electrode spacing (ab/2): note where potential electrodes $(\mathrm{mn} / 2)$ were expanded at individual current electrode stations, there are two V. I, and apparent resistivity values separated by a slash to denote two readings recorded at these stations.

\section{SUMMARY}

Ninety-three vertical electrical-resistivity soundings were made in the Lower Peninsula of Michigan from June through October 1987. The direct-current electrical-resistivity method employing the Schlumbergerelectrode array was used for all soundings. These sounding data were modeled using programs developed by Zohdy (1973) and Zohdy and Bisdorf (written commun., 1987). Raw sounding data. smoothed field data, and results of inverse models are presented. The inverse-model results are generalized using various three- and four-layer models. These generalizations, a summary of near-surface conditions and depths to geologic units (subsurface resistors and conductors) are presented for all soundings. 


\section{REFERENCES CITED}

Mandle, R. J., and Westjohn, D. B., 1987, Preliminary interpretation of vertical electrical-resistivity soundings in the Saginaw Valley. Michigan: U.S. Geological Survey Open-File Report, 87-474, 45 p.

Westjohn, D. B., 1989, Application of geophysics in the delineation of the freshwater/saline-water interface in the Michigan basin: Symposium volume, 24th Annual American Water Resources Association (in press).

Zohdy, A. A. R., Eaton, G. P., and Mabey, D. R., 1974, Application of surface geophysics to ground-water investigations: U.S. Geological Survey Techniques of Water-Resources Investigations, book 2 . chap. D1: p. 5-66.

Zohdy, A. A. R., 1973, A computer program for the automatic interpretation of Schlumberger sounding curves over horizontally stratified media: available only from U.S. Department of Commerce. National Technical Information Service, Springfield, VA 22161, as U.S. Geological Survey Report USGS-GD-74-017, PB232 703, 25 p. 


\section{Table 1.-- Summary of observations for vertical}

electrical-resistivity soundings

Survey: The prefix, VES should be added to all survey numbers listed in this table.

Near-surface conditions: D, dry, sandy, well drained soil: W. partially or fully-saturated till or lacustrine materials.

Curve type: See text for explanation of different curve types.

Maximum $\mathrm{AB} / 2$ : Distance, in meters, between a current electrode and center of Schlumberger electrode arrangement.

Stations deleted: The number of apparent resistivity values that were deleted before computer processing of data.

Depth to geologic unit: In meters below land surface.

Geologic unit: Mmf, Mississippian Michigan Formation: Mmss, Mississippian Marshall Sandstone: Mcws, Mississippian Coldwater Shale; NP, not present: no entry in column for geologic unit indicates data not available.

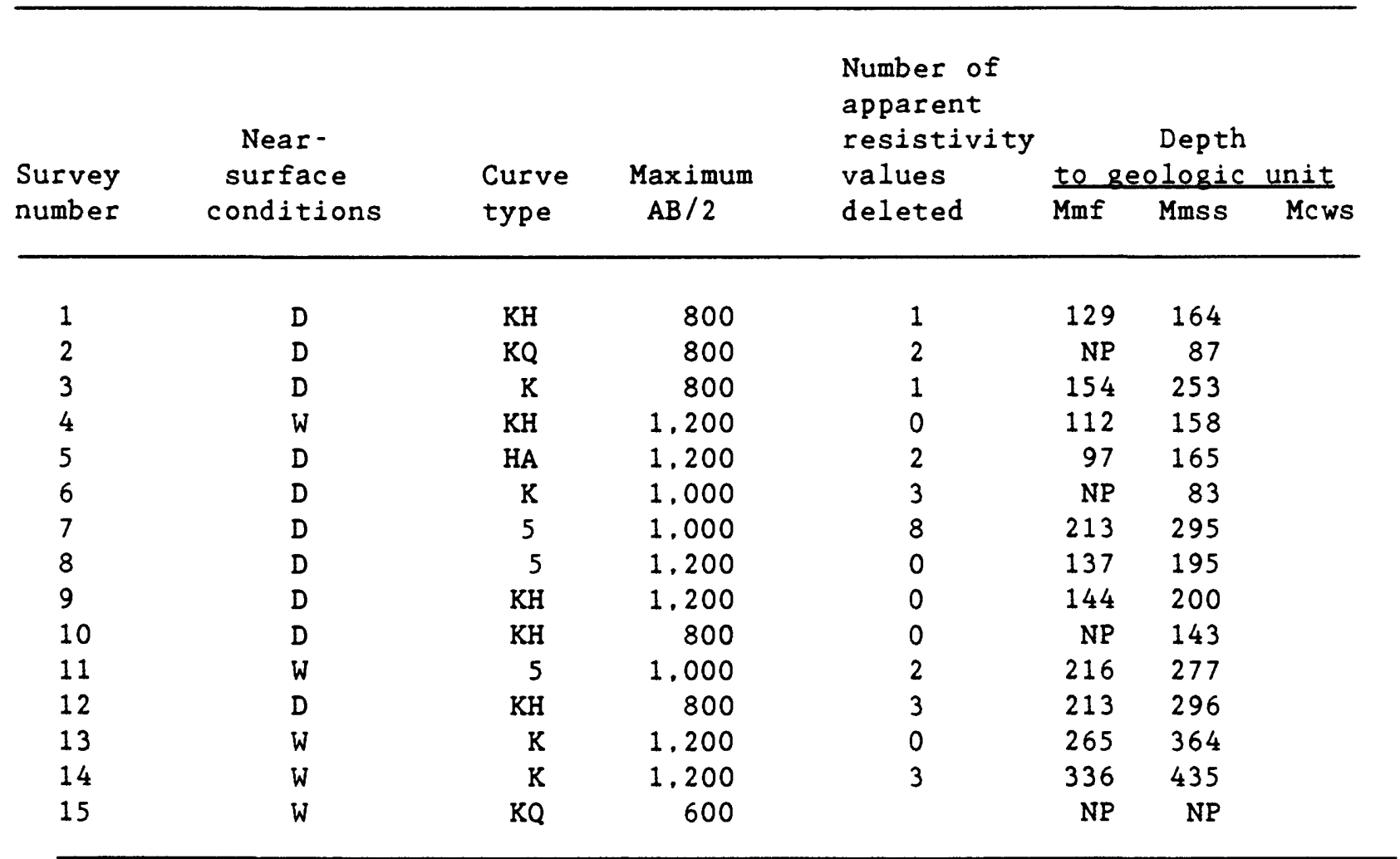


Table 1.--Summary of observations for vertical electrical-resistivity soundings--Continued

\begin{tabular}{|c|c|c|c|c|c|c|c|}
\hline $\begin{array}{l}\text { Survey } \\
\text { number }\end{array}$ & $\begin{array}{c}\text { Near- } \\
\text { surface } \\
\text { conditions }\end{array}$ & $\begin{array}{l}\text { Curve } \\
\text { type }\end{array}$ & $\begin{array}{c}\text { Maximum } \\
\mathrm{AB} / 2\end{array}$ & $\begin{array}{l}\text { Number of } \\
\text { apparent } \\
\text { resistivity } \\
\text { values } \\
\text { deleted }\end{array}$ & $\frac{\text { to } 8}{\operatorname{Mmf}}$ & $\begin{array}{l}\text { Depth } \\
\text { olooic } \\
\text { Mmss }\end{array}$ & $\frac{\text { unit }}{\text { Mcws }}$ \\
\hline 16 & D & $\mathrm{K}$ & 600 & 0 & 259 & 371 & \\
\hline 17 & D & HK & 1.200 & 3 & 259 & 371 & \\
\hline 18 & $W$ & 5 & 1.200 & 4 & 128 & 161 & \\
\hline 19 & W & HK & 1.000 & 1 & 314 & 543 & \\
\hline 20 & D & 5 & 1.400 & 3 & 291 & 456 & \\
\hline 21 & W & HA & 1.400 & 0 & 272 & 370 & \\
\hline 22 & W & 5 & 1.200 & 0 & 274 & 382 & \\
\hline 23 & D & $\mathrm{QH}$ & 1.000 & 1 & 196 & 250 & \\
\hline 24 & W & 6 & 1.000 & 1 & 197 & 250 & \\
\hline 25 & W & $\mathrm{KH}$ & 1,200 & 0 & $\mathrm{NP}$ & 117 & \\
\hline 26 & W & 5 & 600 & 2 & 266 & 399 & \\
\hline 27 & D & $\mathrm{KH}$ & 1,200 & 2 & 272 & 370 & \\
\hline 28 & D & 5 & 1.000 & 11 & 156 & 259 & \\
\hline 29 & D & $\mathrm{H}$ & 1.200 & 2 & 131 & 222 & \\
\hline 30 & D & 6 & 800 & 0 & $\mathrm{NP}$ & 146 & \\
\hline 31 & W & HK & 800 & 0 & $\mathrm{NP}$ & 93 & \\
\hline 32 & W & 5 & 1.200 & 0 & 124 & 142 & \\
\hline 33 & D & HK & 600 & 0 & $\mathrm{NP}$ & $\mathrm{NP}$ & 189 \\
\hline 34 & D & $\mathrm{KH}$ & 1.200 & 3 & 275 & 454 & \\
\hline 35 & D & $\mathrm{KH}$ & 1.000 & 2 & 136 & 199 & \\
\hline 36 & D & HK & 800 & 3 & $\mathrm{NP}$ & NP & 148 \\
\hline 37 & $\mathrm{~W}$ & $\mathrm{HK}$ & 800 & 0 & $\mathrm{NP}$ & NP & 213 \\
\hline 38 & $D$ & 6 & 1.000 & 0 & $\mathrm{NP}$ & NP & 167 \\
\hline 39 & W & 6 & 1,400 & 1 & $\mathrm{NP}$ & NP & 167 \\
\hline 40 & W & $\mathrm{K}$ & 600 & 2 & 184 & 269 & \\
\hline 41 & D & 6 & 1.000 & 3 & 298 & 437 & \\
\hline 42 & D & 5 & 600 & 3 & 219 & 410 & \\
\hline 43 & W & 5 & 1.200 & 0 & 133 & 201 & \\
\hline 44 & W & $\mathrm{KH}$ & 1.200 & 0 & 296 & 444 & \\
\hline 45 & D & $\mathrm{HA}$ & 1.200 & 3 & $\mathrm{NP}$ & 115 & \\
\hline 46 & D & $\mathrm{QH}$ & 1.200 & 0 & $\mathrm{NP}$ & 109 & \\
\hline 47 & W & 5 & 1.200 & 0 & 344 & NP & \\
\hline 48 & D & HK & 800 & 2 & $\mathrm{NP}$ & 21 & \\
\hline 49 & W & 5 & 800 & 3 & 104 & 169 & \\
\hline 50 & W & $\mathrm{KH}$ & 1.200 & 0 & 104 & 169 & \\
\hline 51 & W & 5 & 800 & 0 & 27 & 92 & \\
\hline 52 & W & HK & 600 & 5 & 20 & 90 & \\
\hline 53 & W & $\mathrm{K}$ & 800 & 0 & NP & 73 & \\
\hline 54 & W & $\mathrm{K}$ & 400 & 0 & $\mathrm{NP}$ & 42 & \\
\hline 55 & W & HK & 600 & 1 & NP & 15 & \\
\hline
\end{tabular}


Table 1.--Summary of observations for vertical electrical-resistivity soundings--Continued

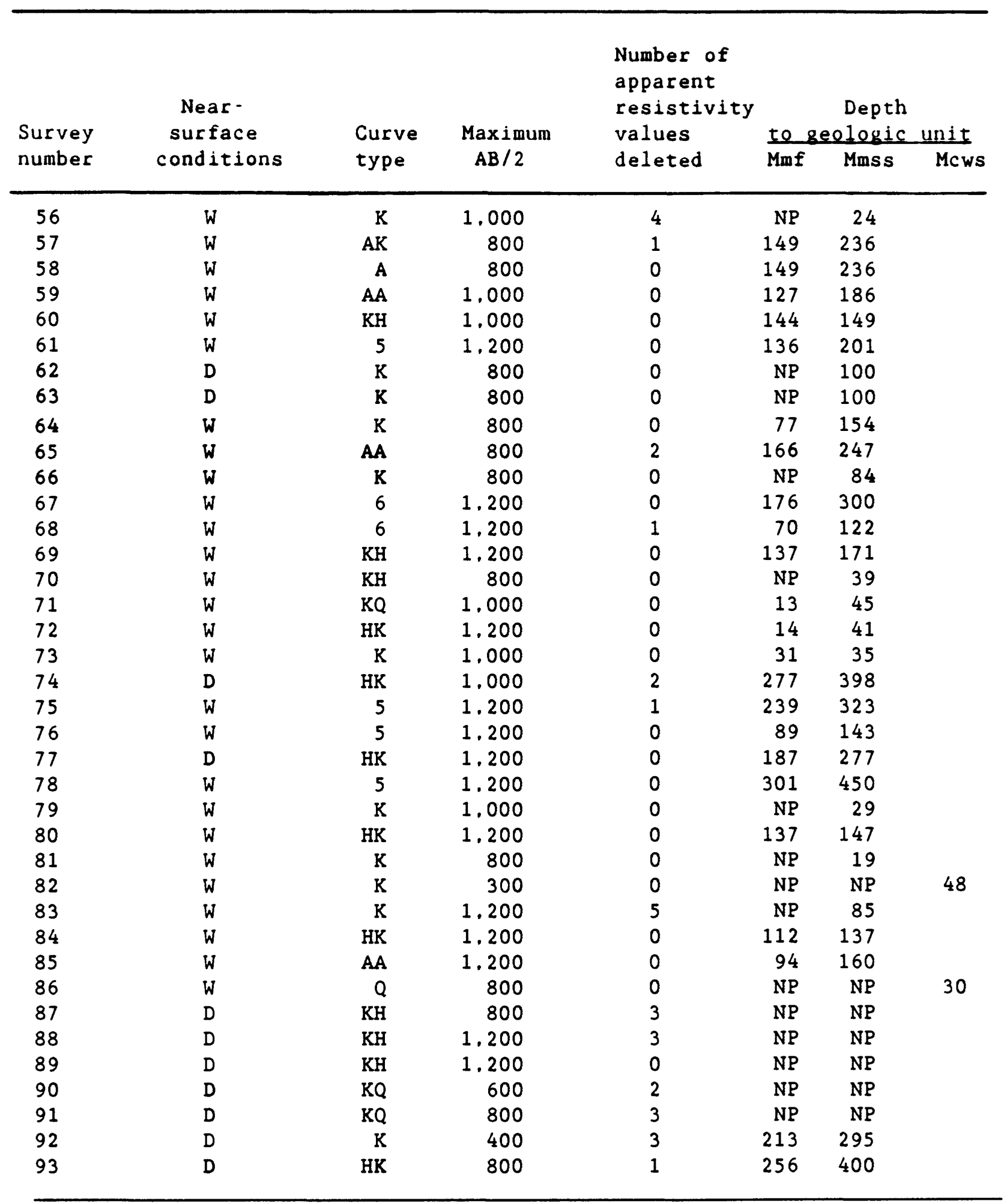




\section{APPENDIX}

Vertical electrical-resistivity sounding data, shifted data, and calculated electric layers for locations in the Lower Peninsula of Michigan. 


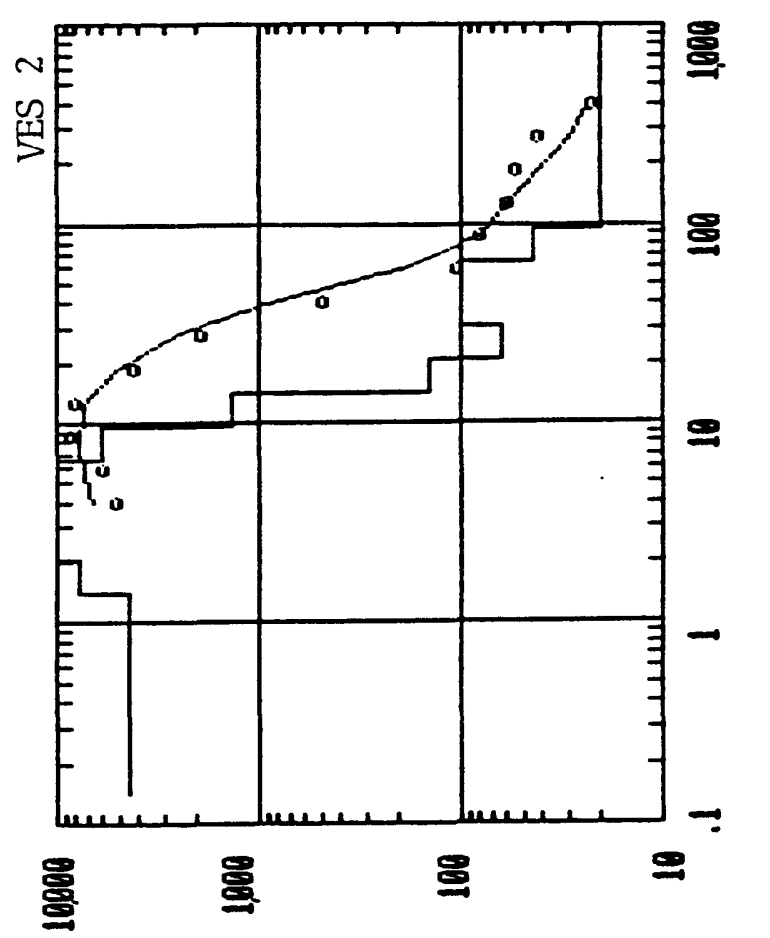

놀

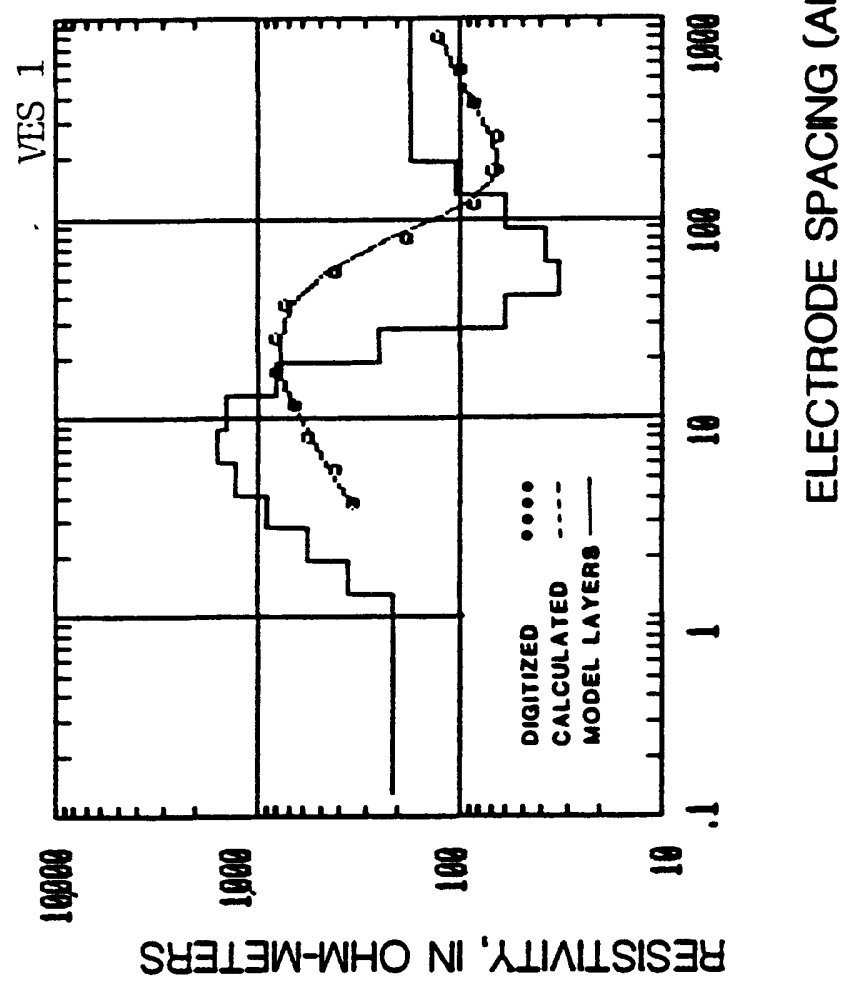

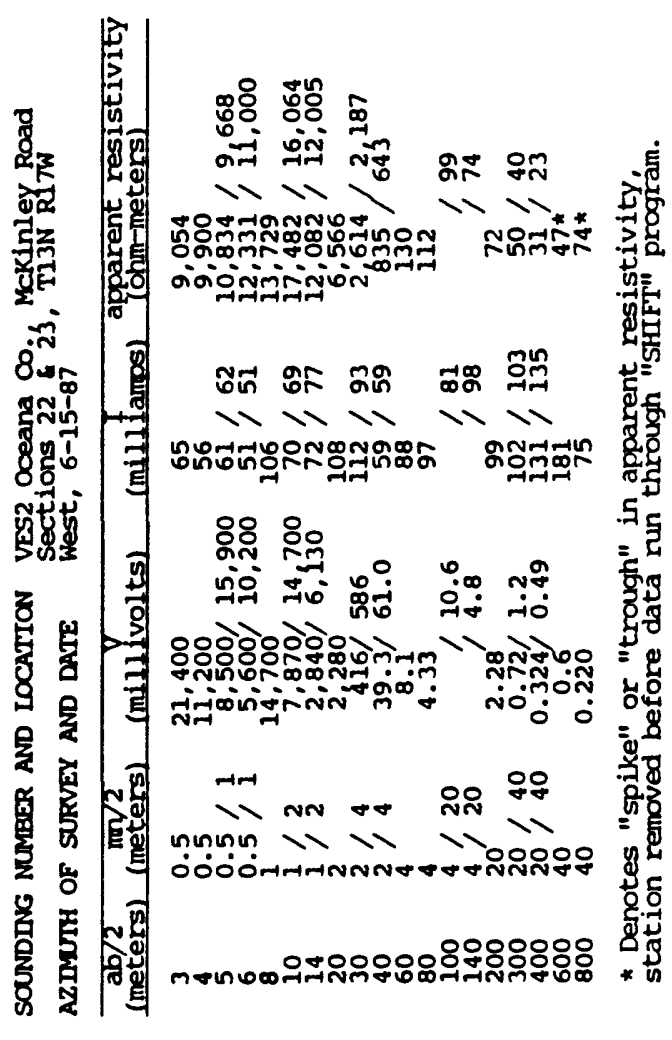

ล

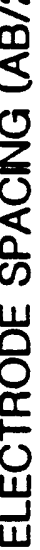

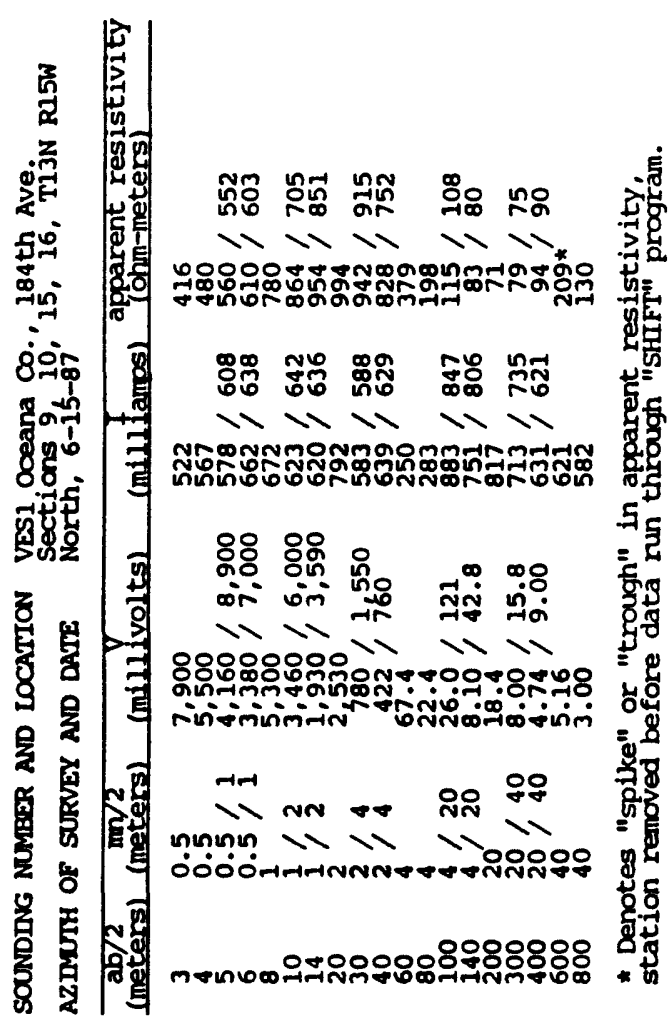



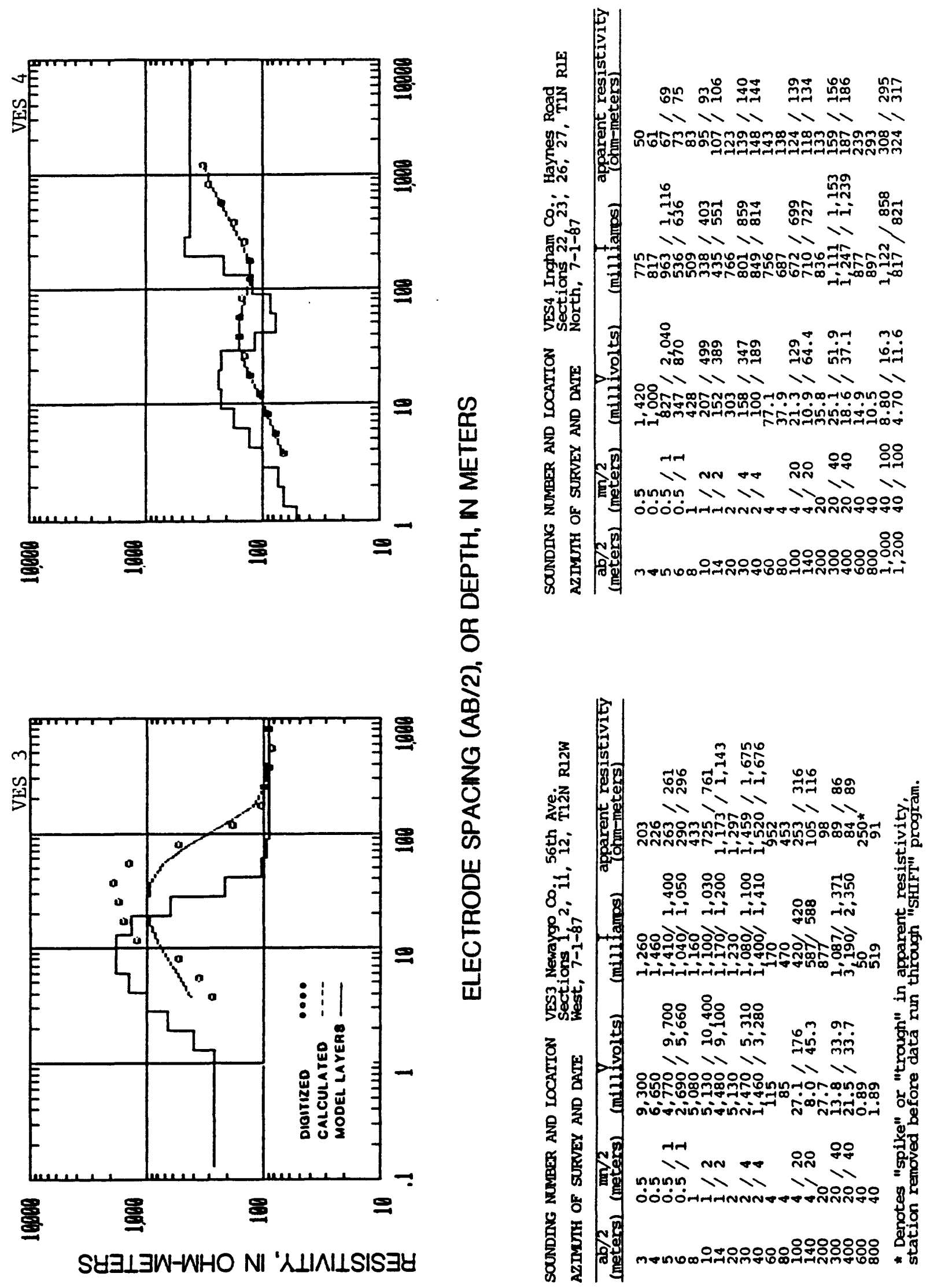

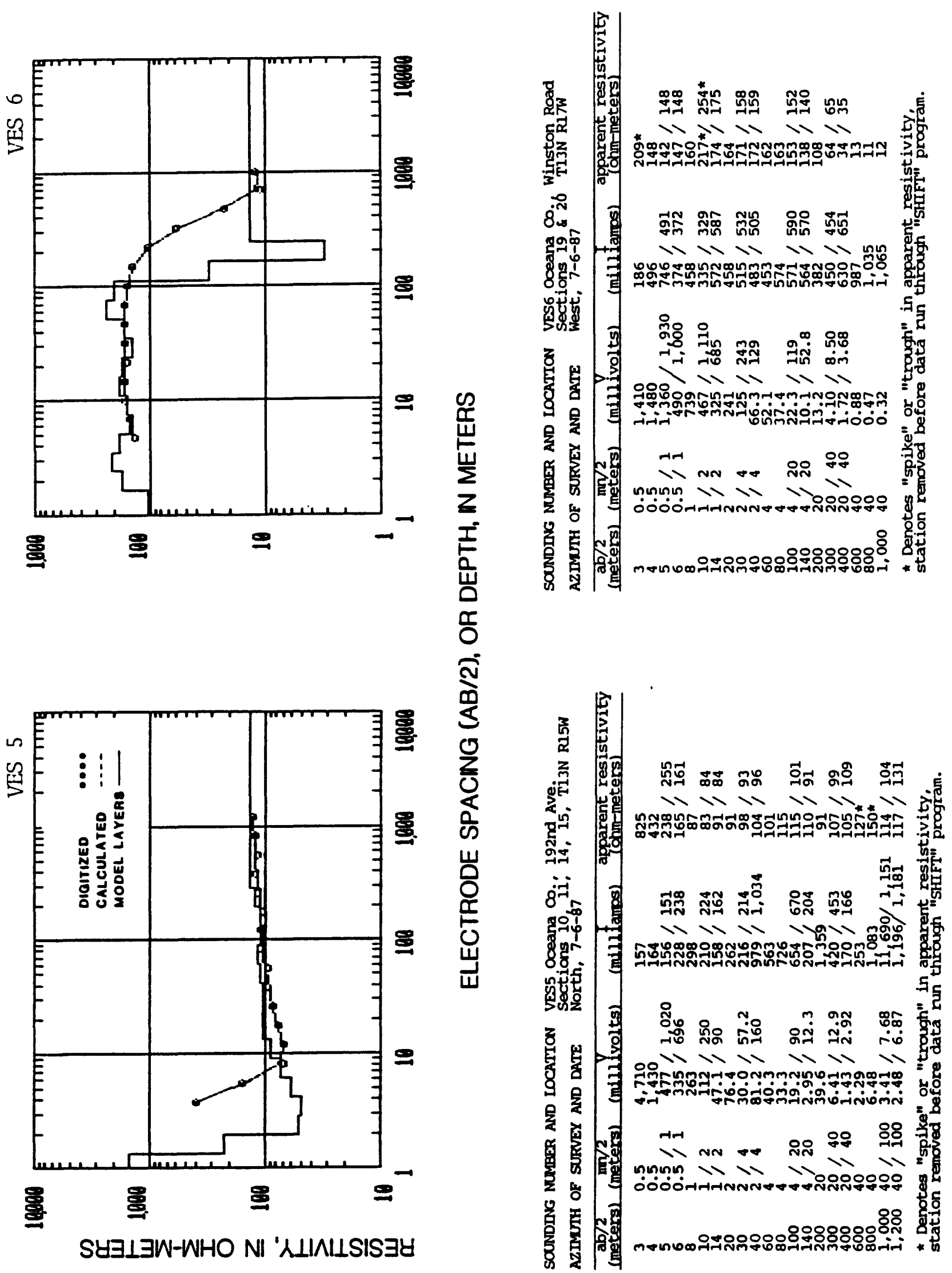

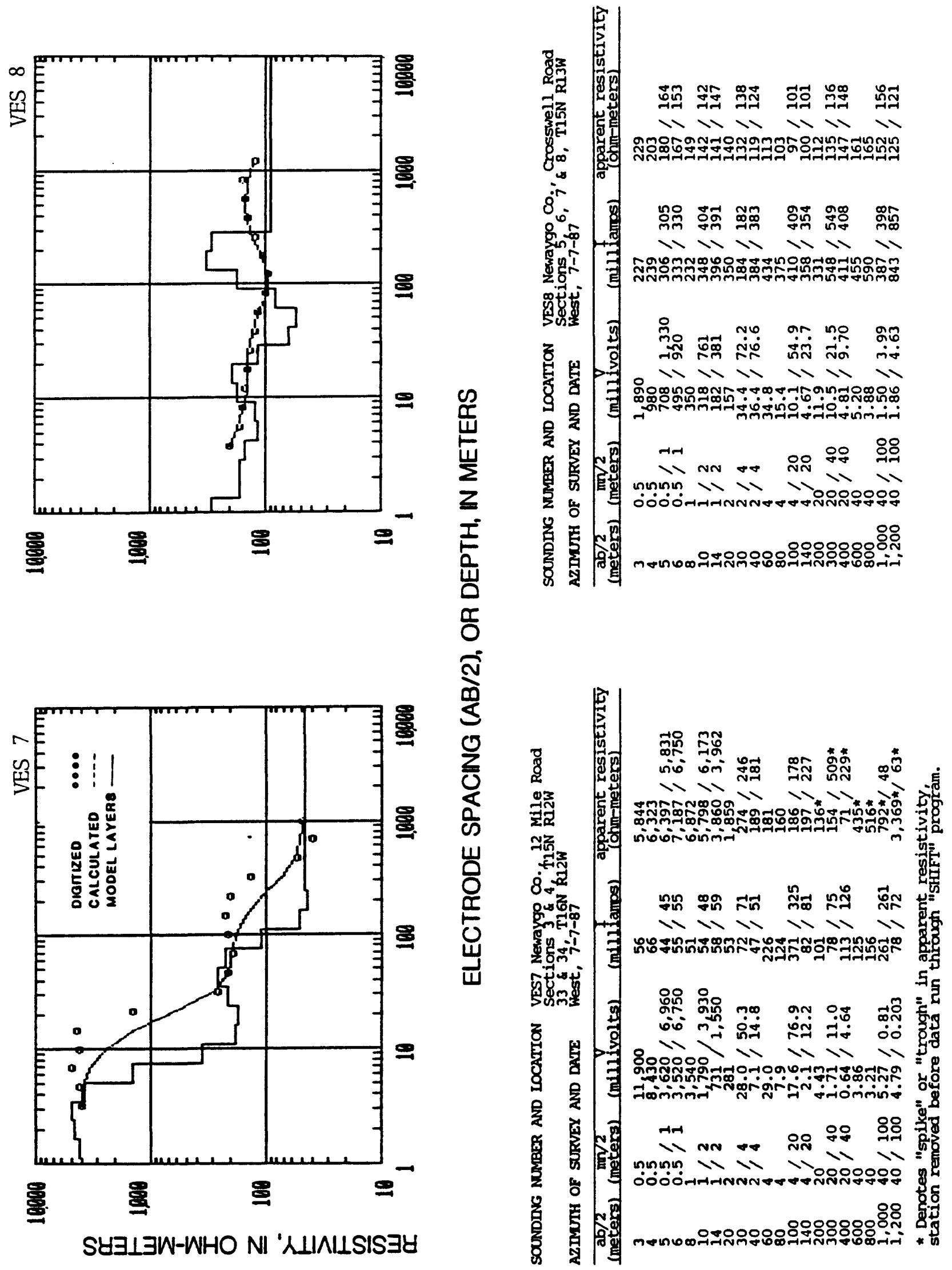

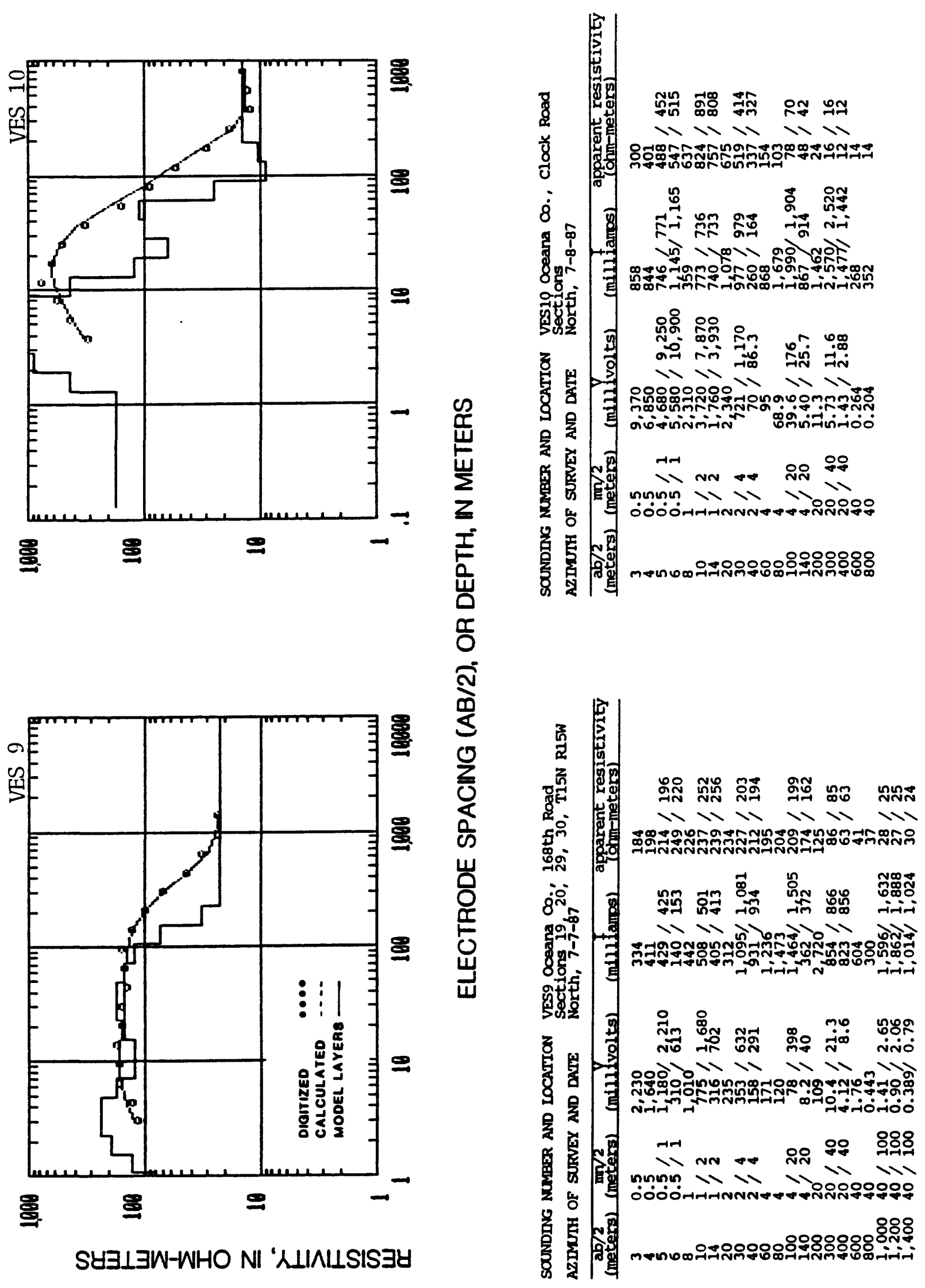

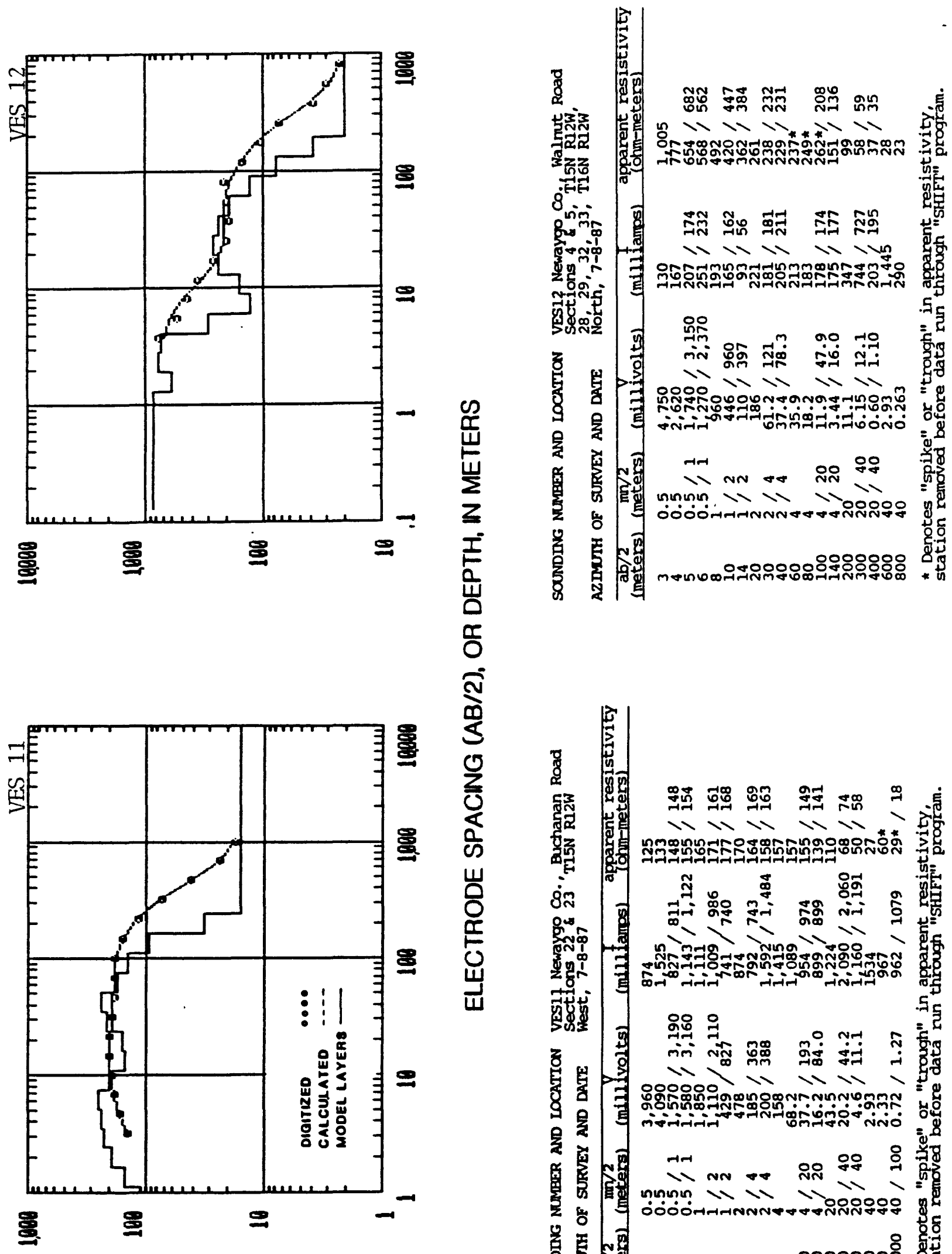

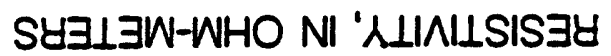

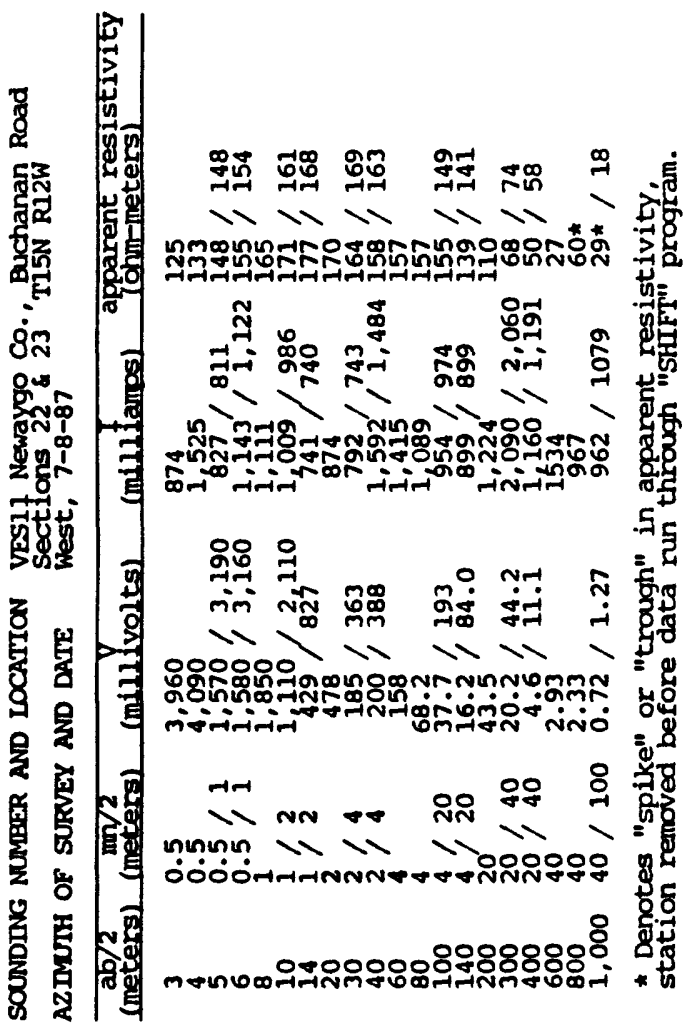



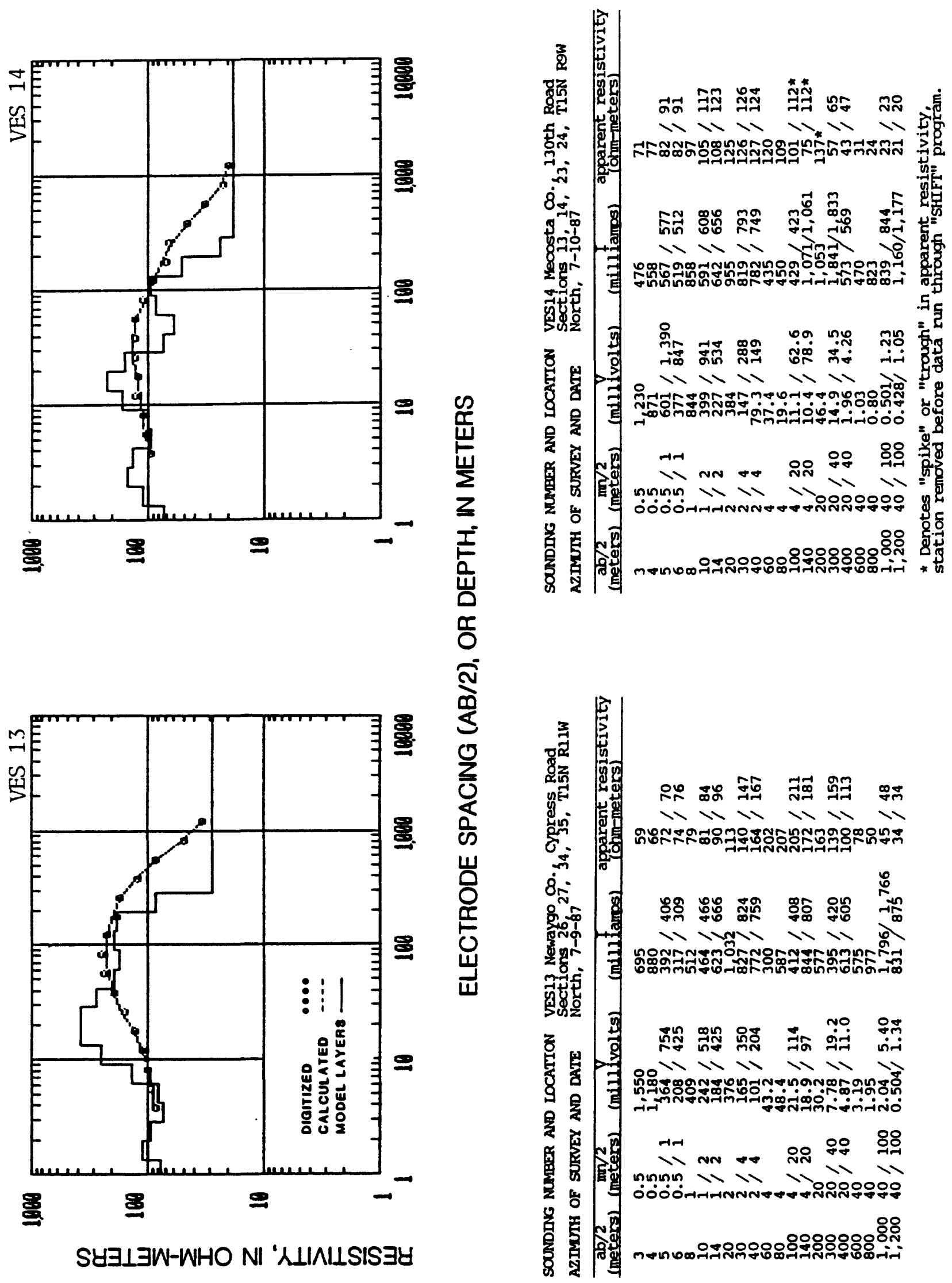


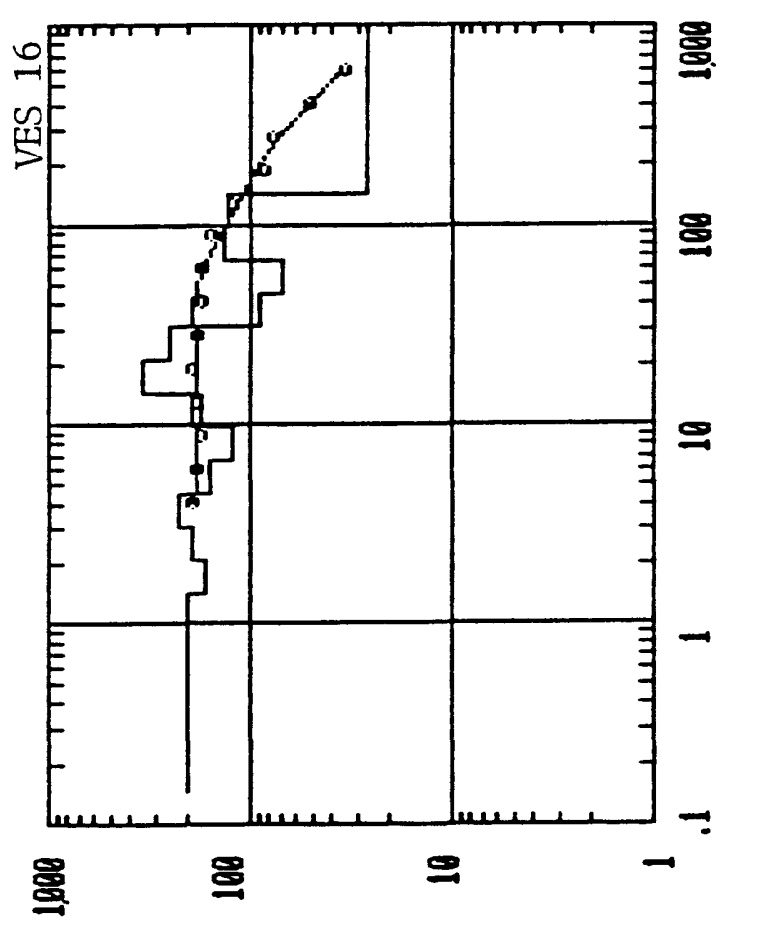

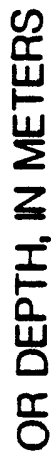

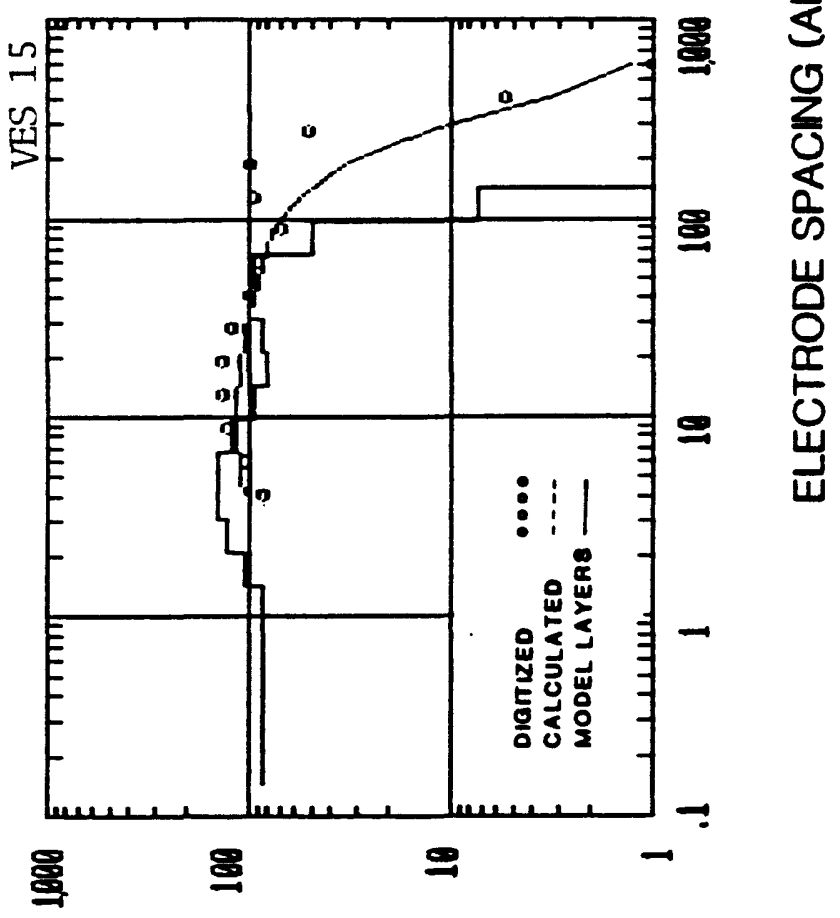

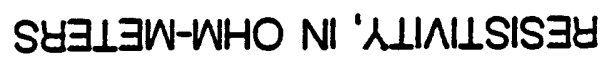
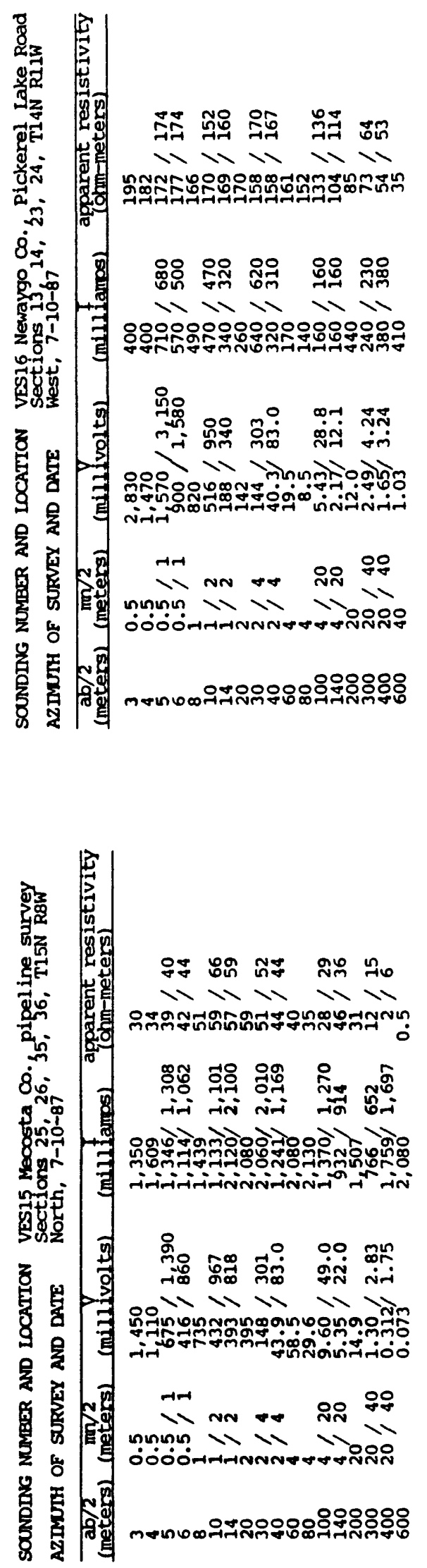

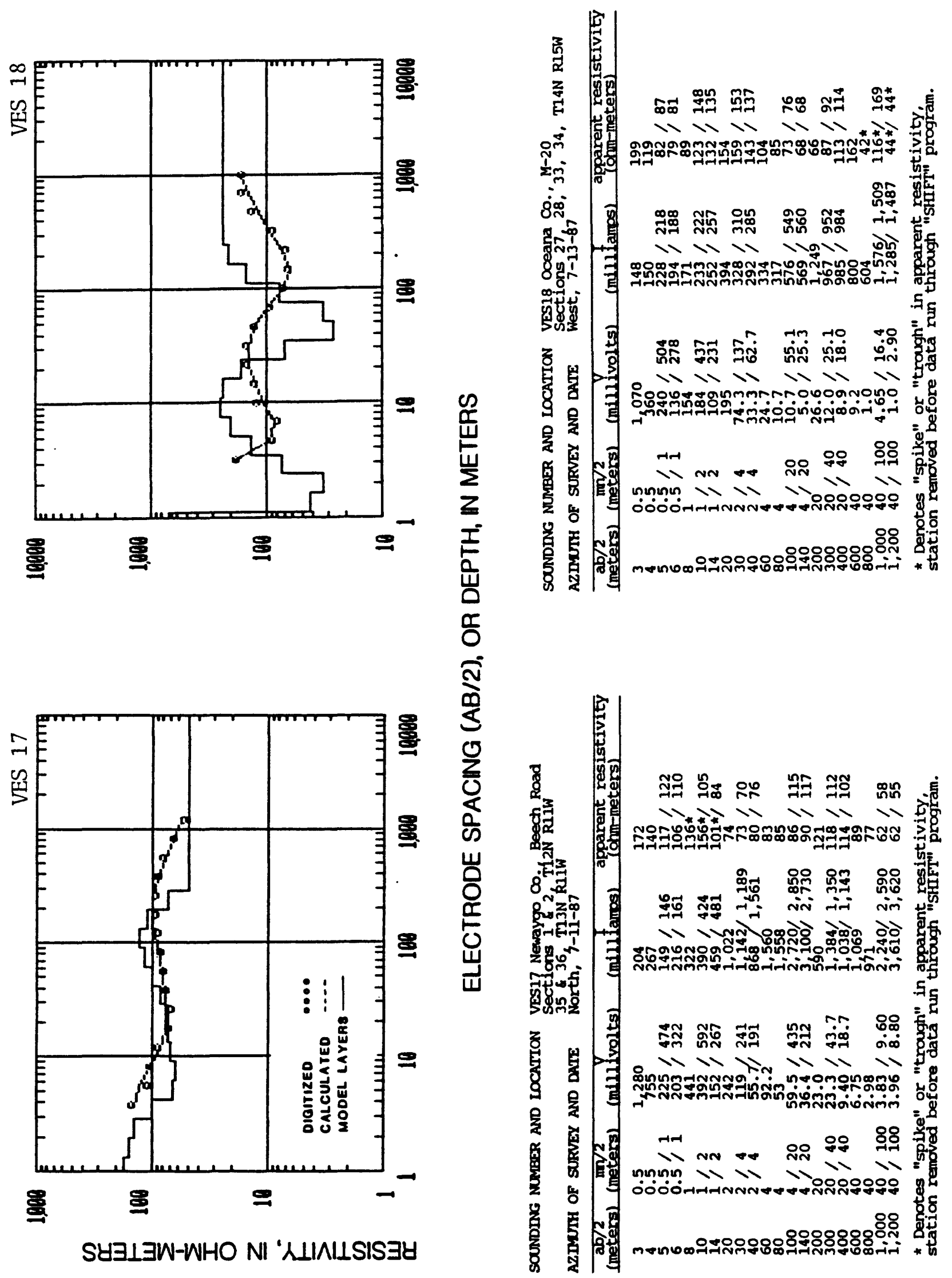

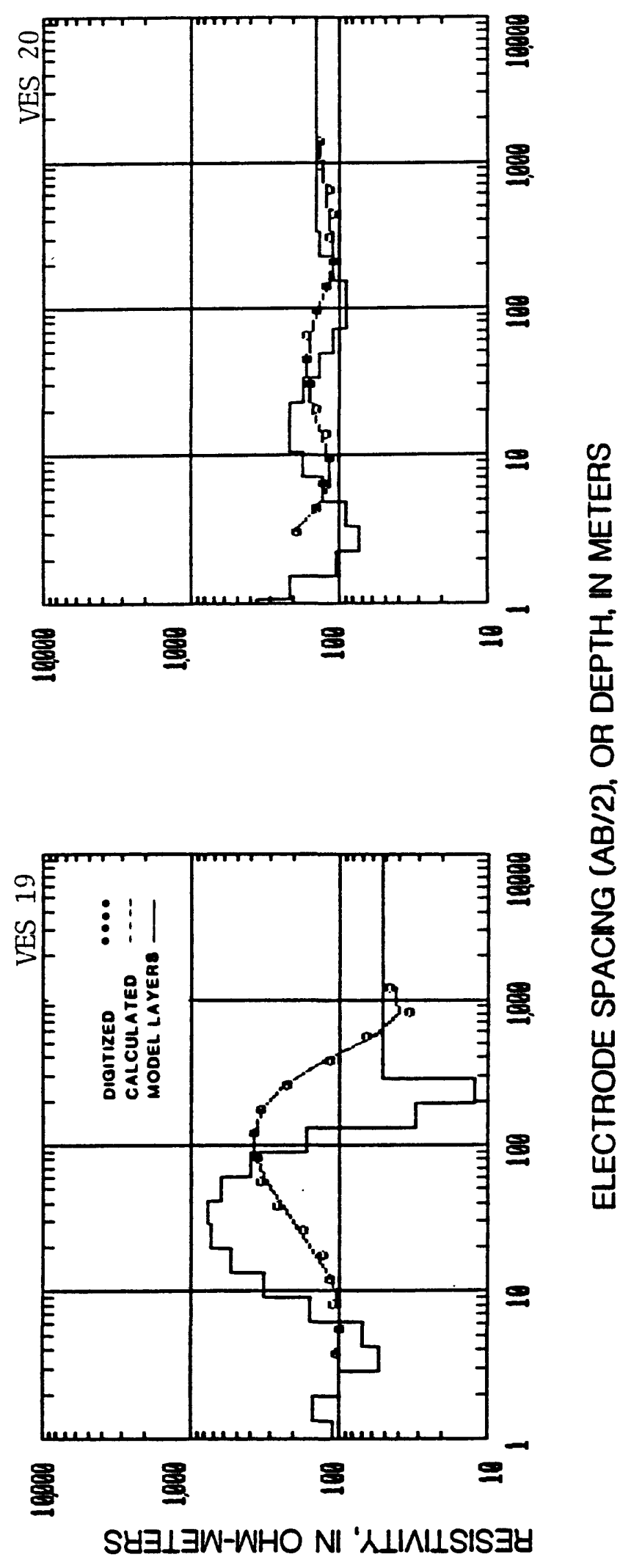

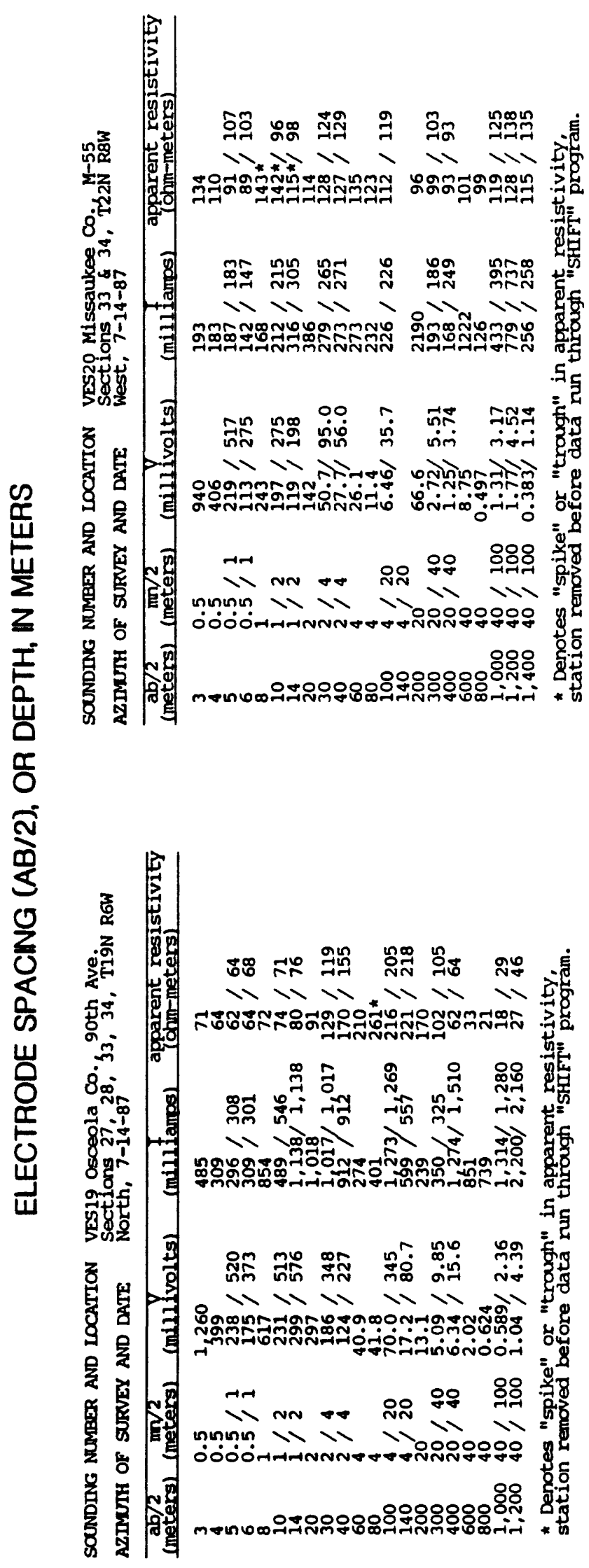



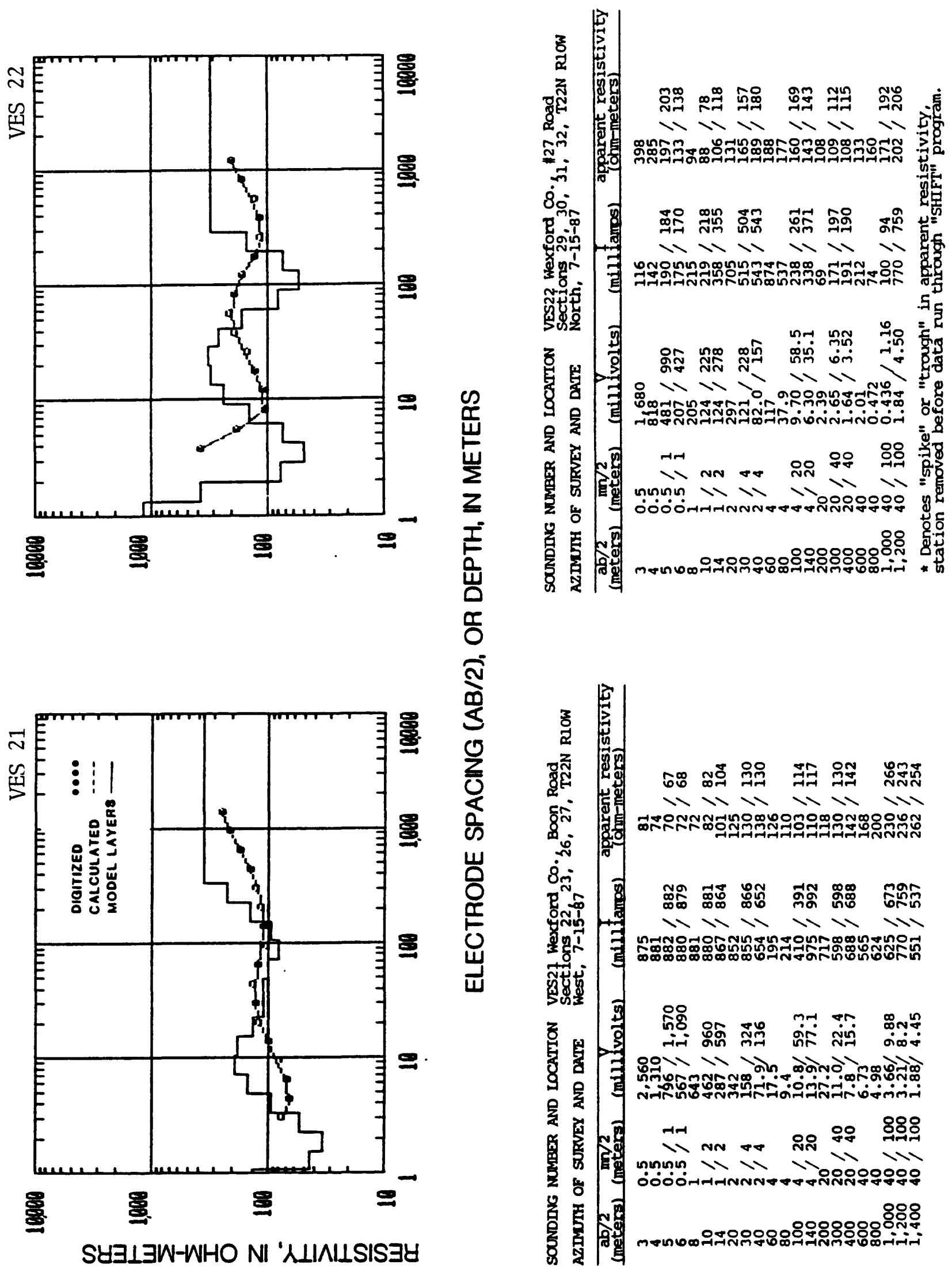

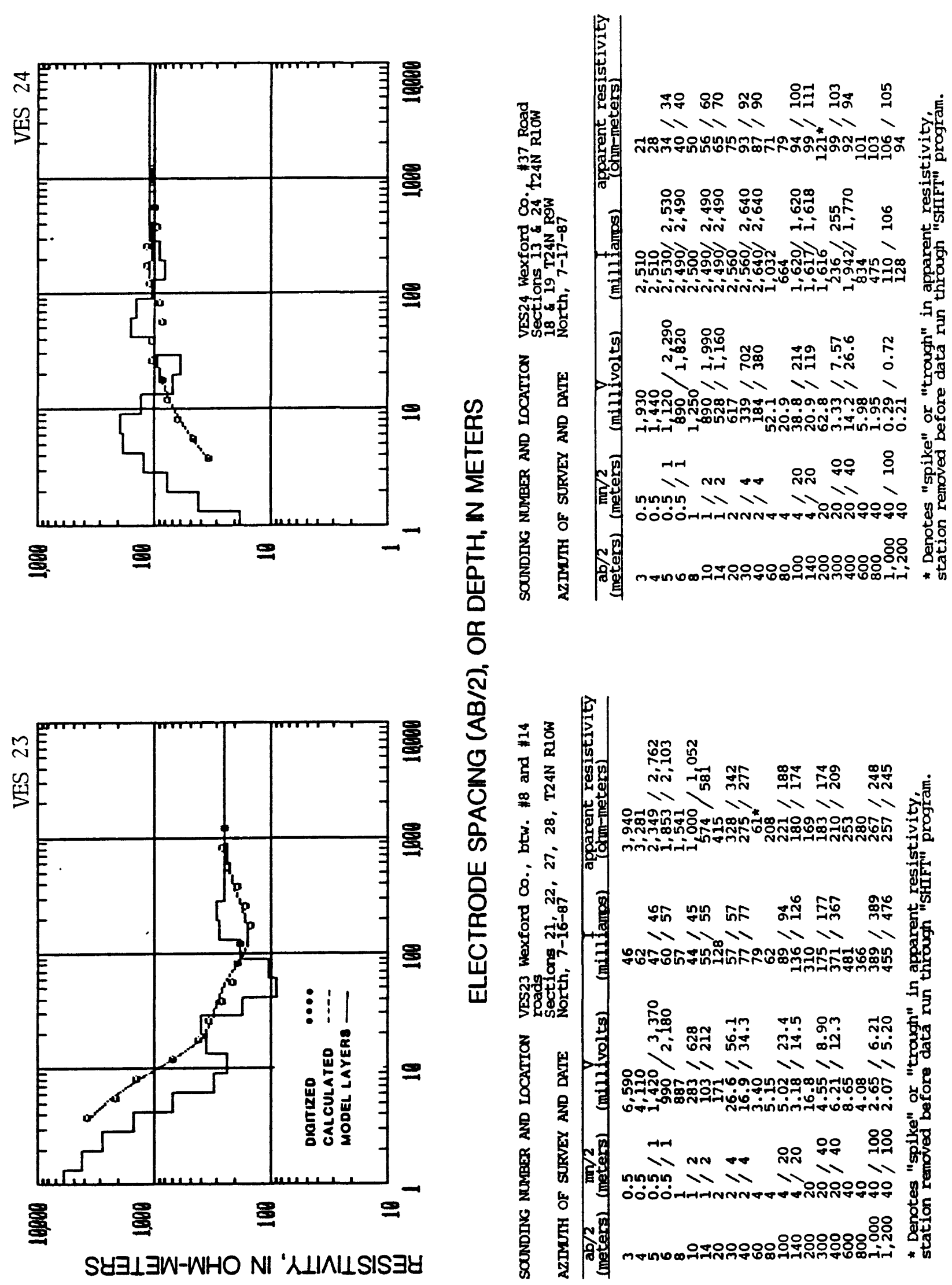

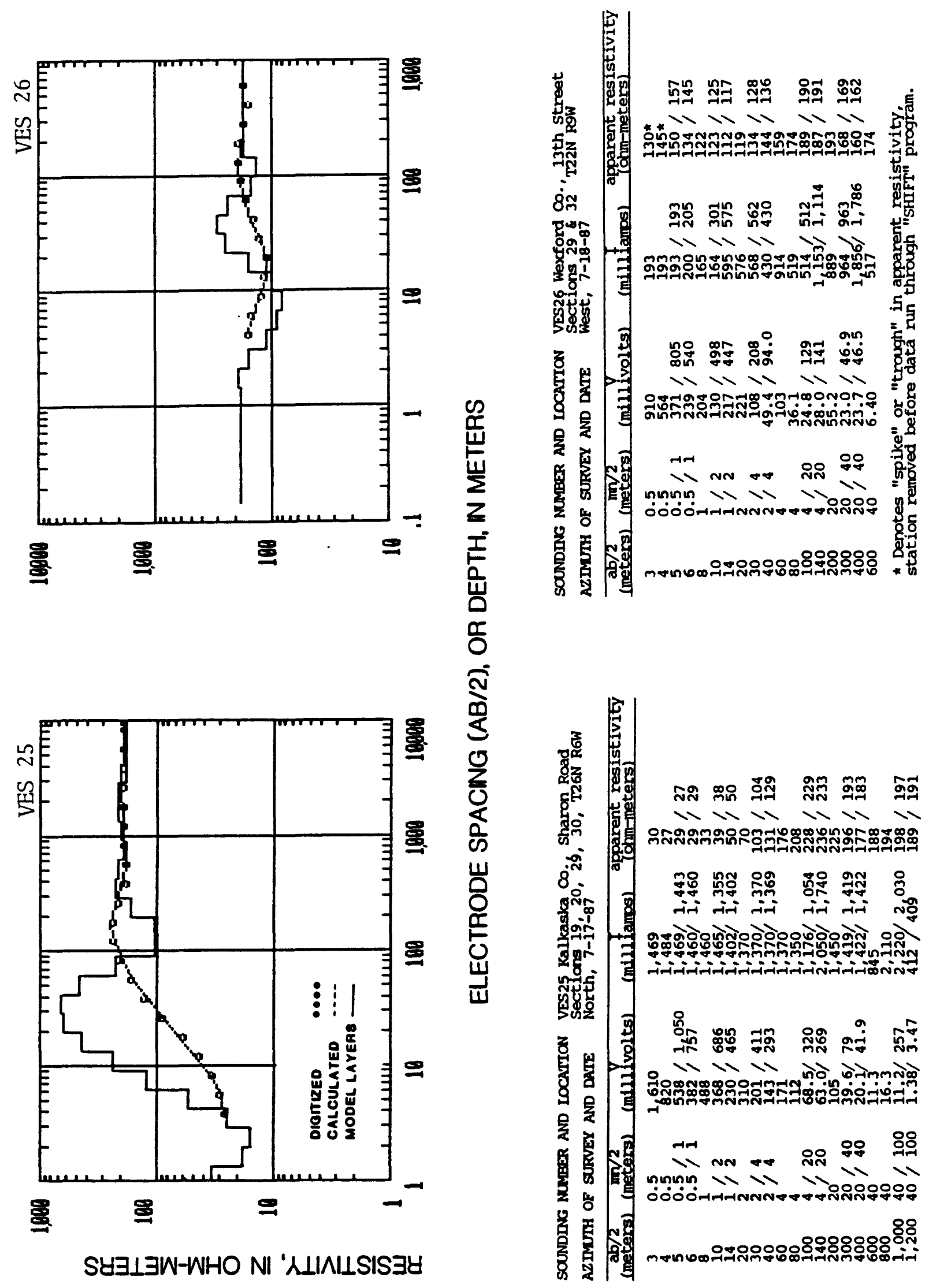

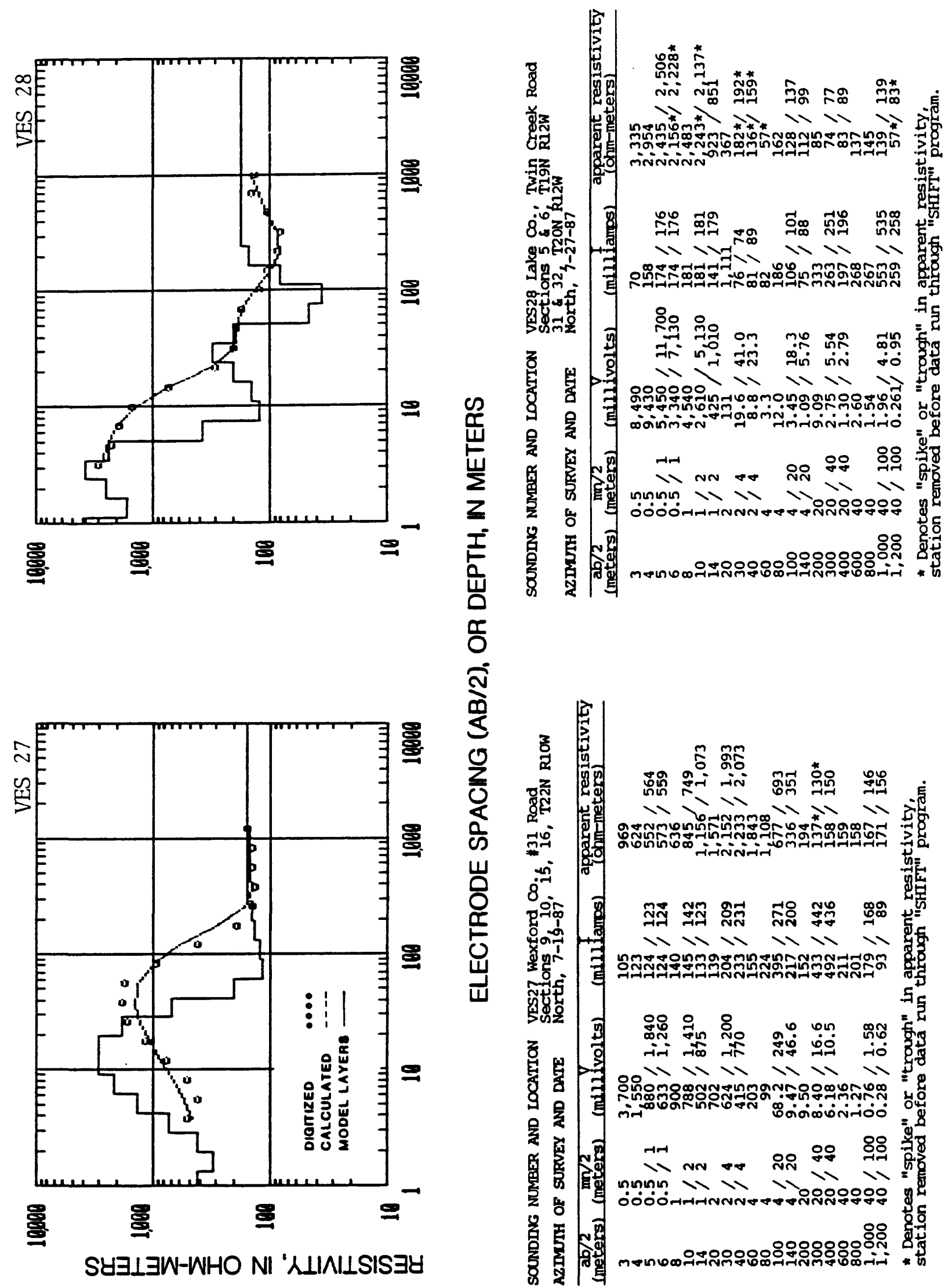

๘

ลิ

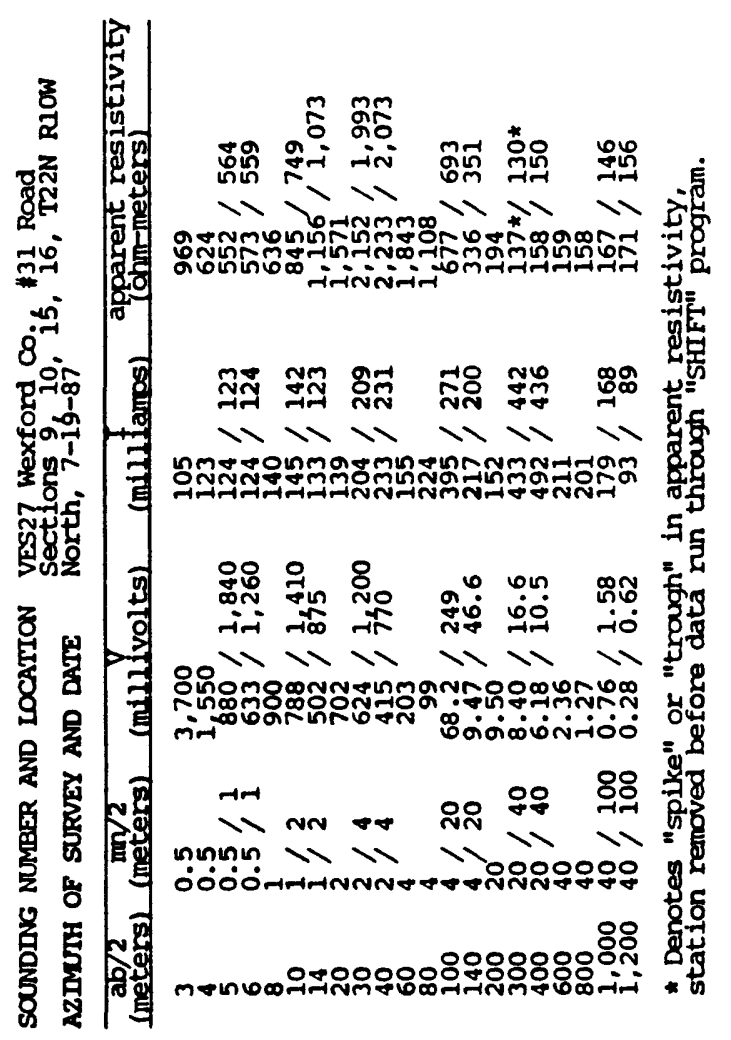




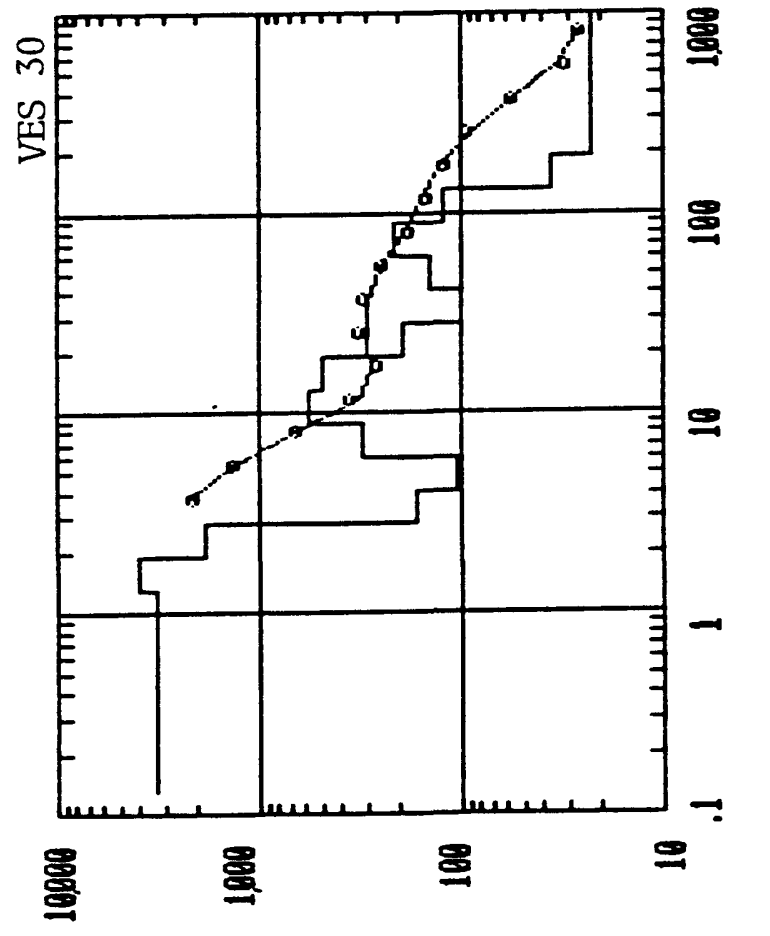

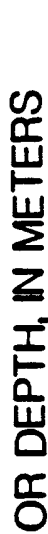

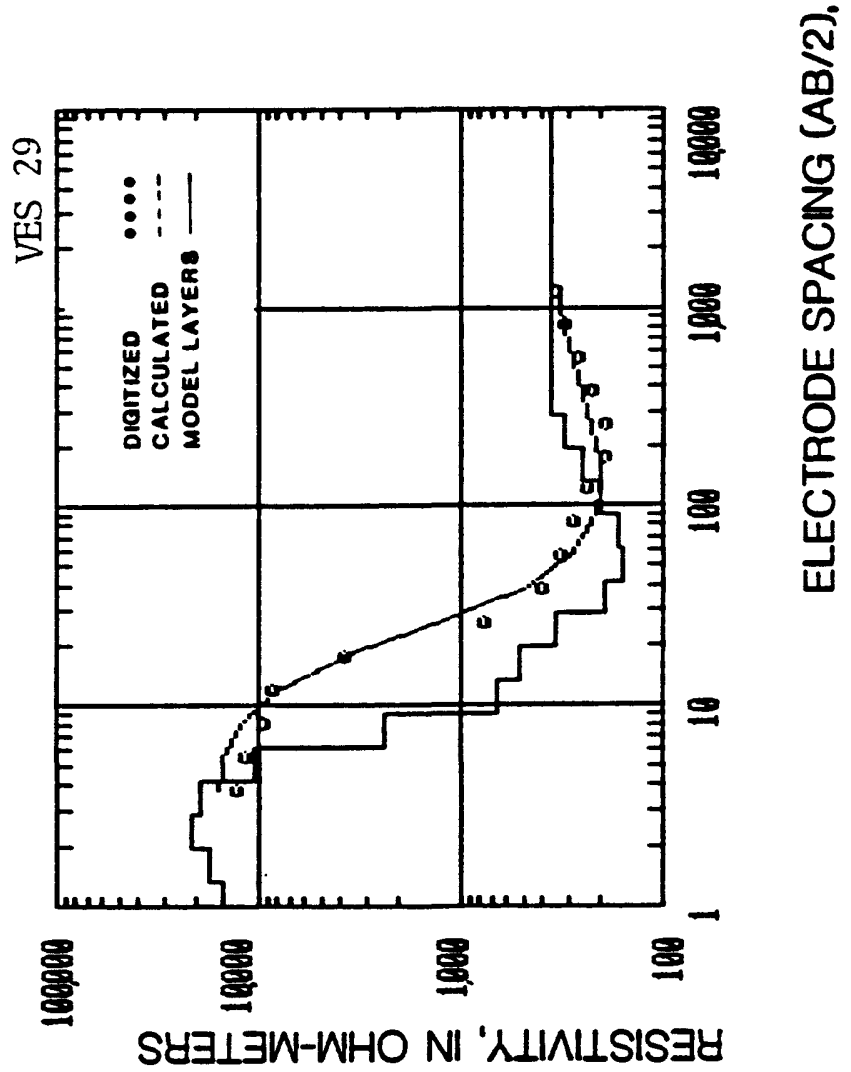

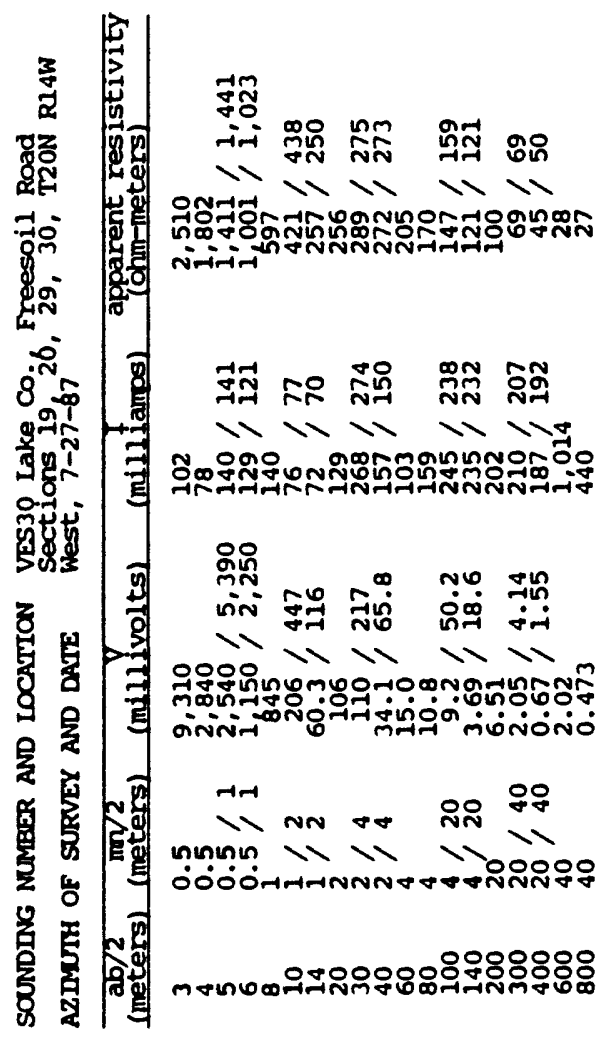

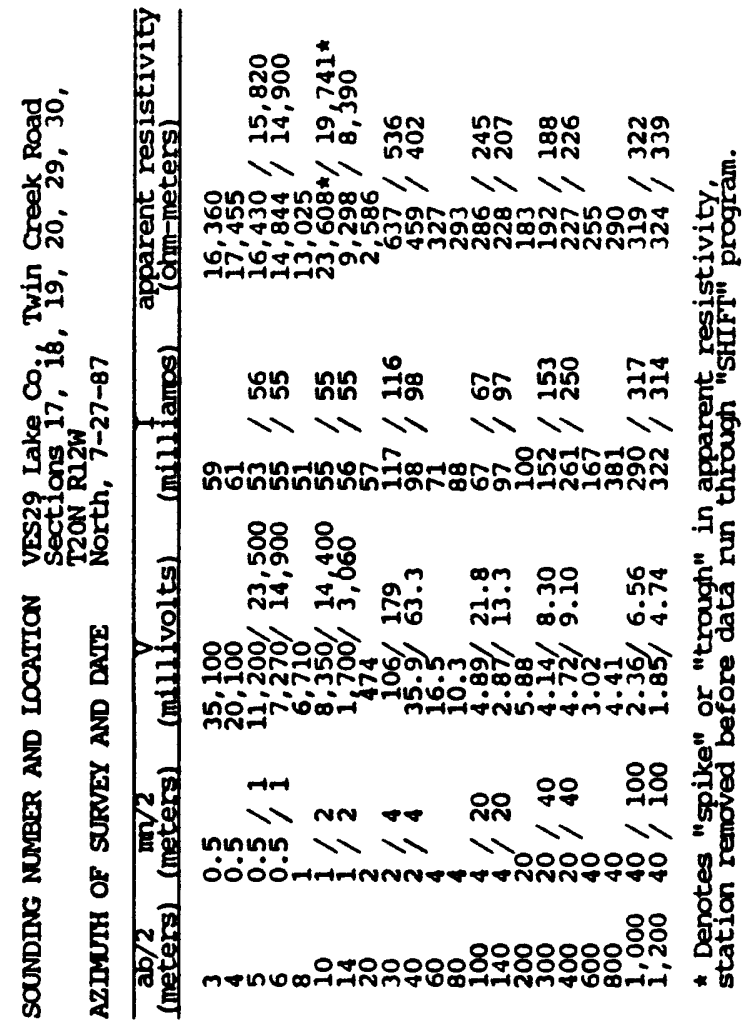




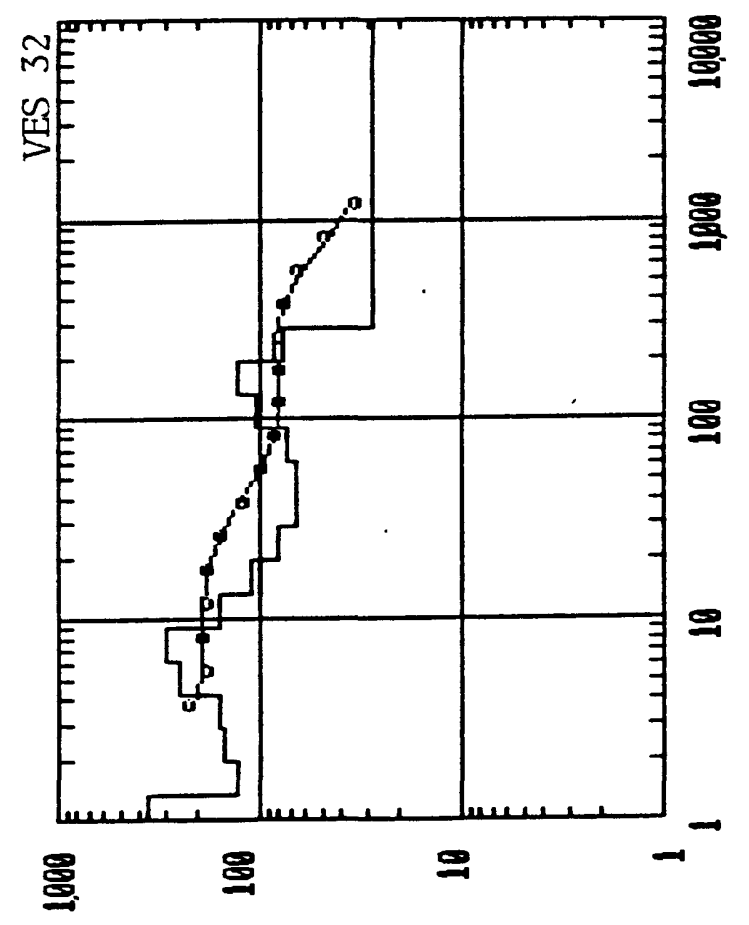

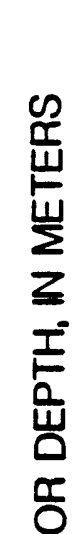

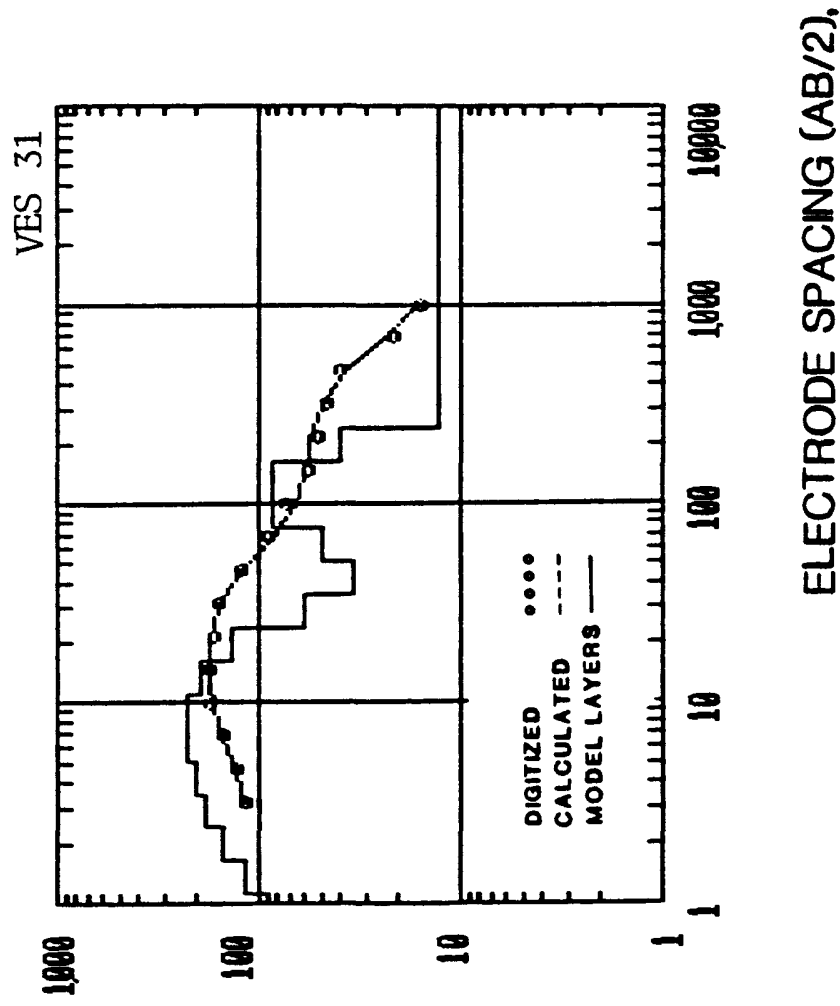

SU $\exists \perp \exists W-W H O N I$ N $\lambda \perp I A I S I S \exists Y$

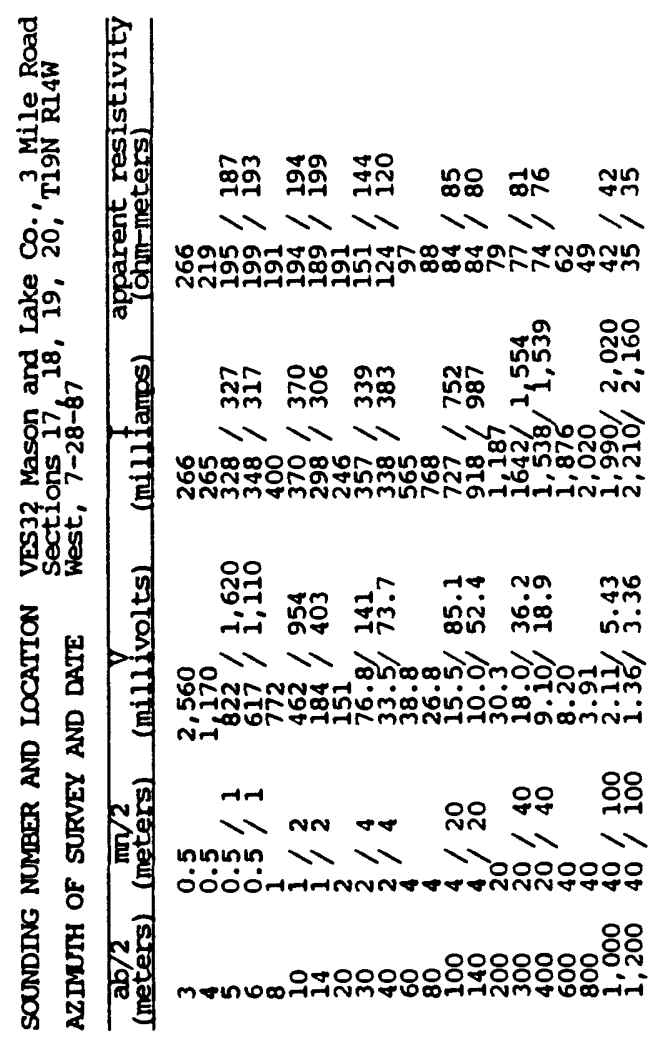
察室

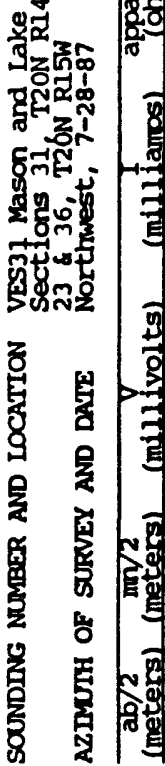

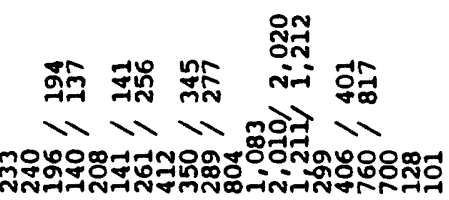

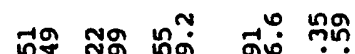

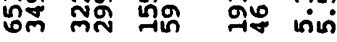

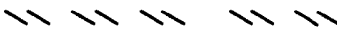
onฒ-1007

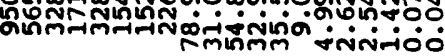

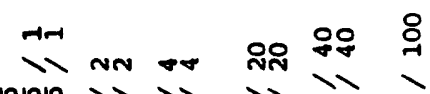

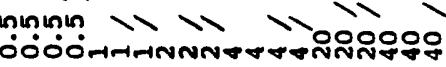
อั00 m-иம 

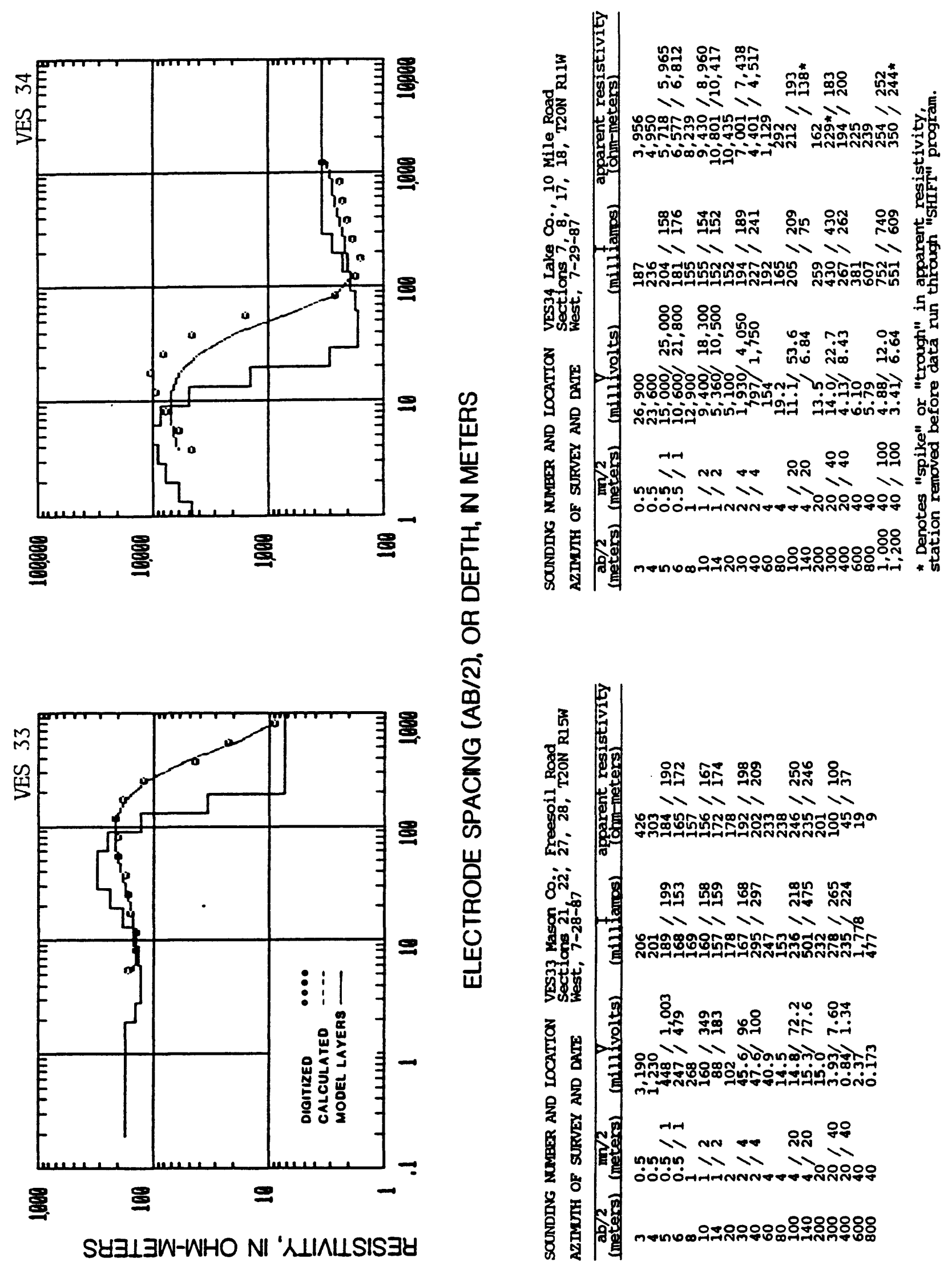

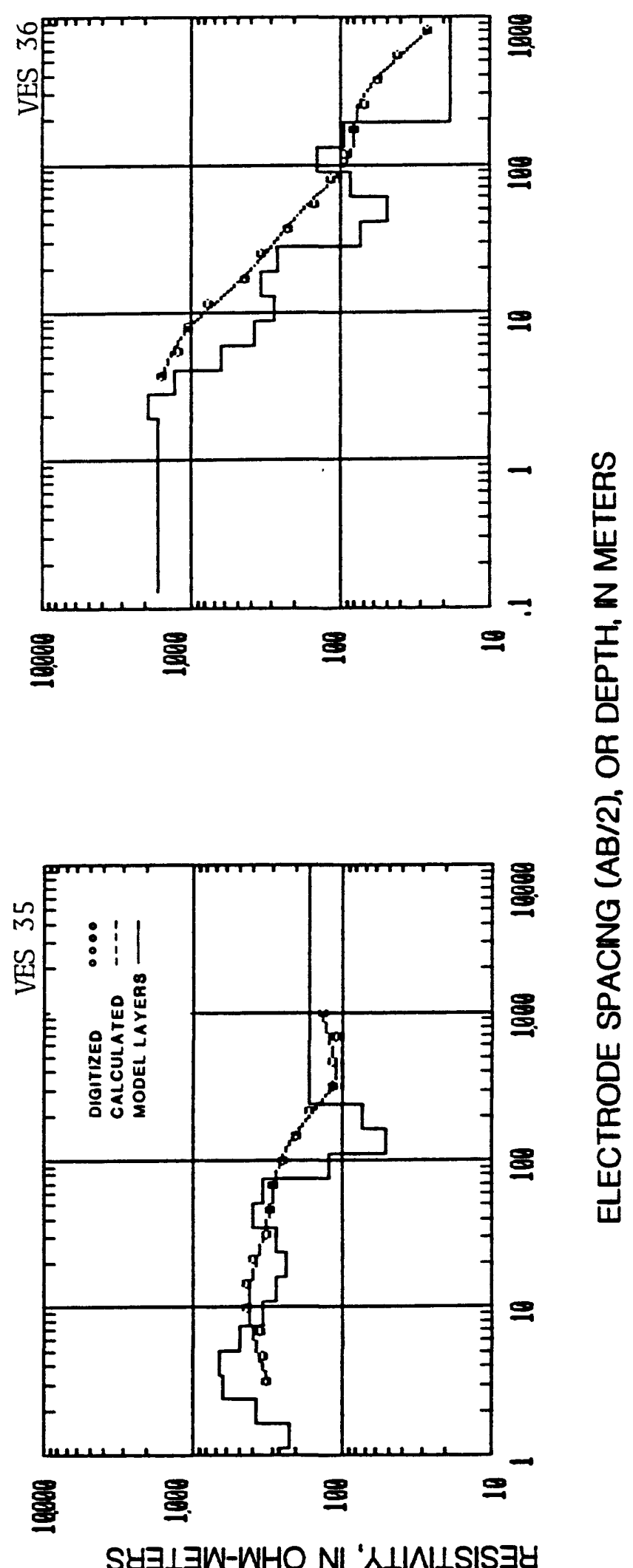

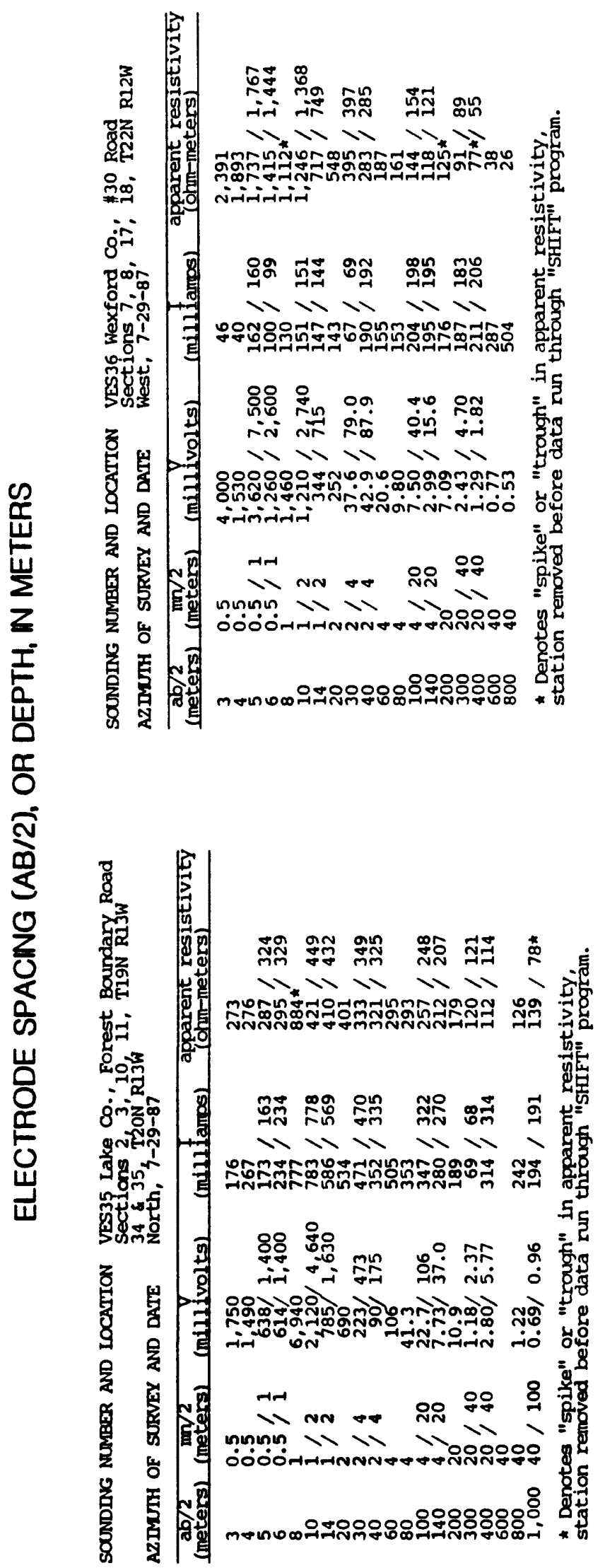




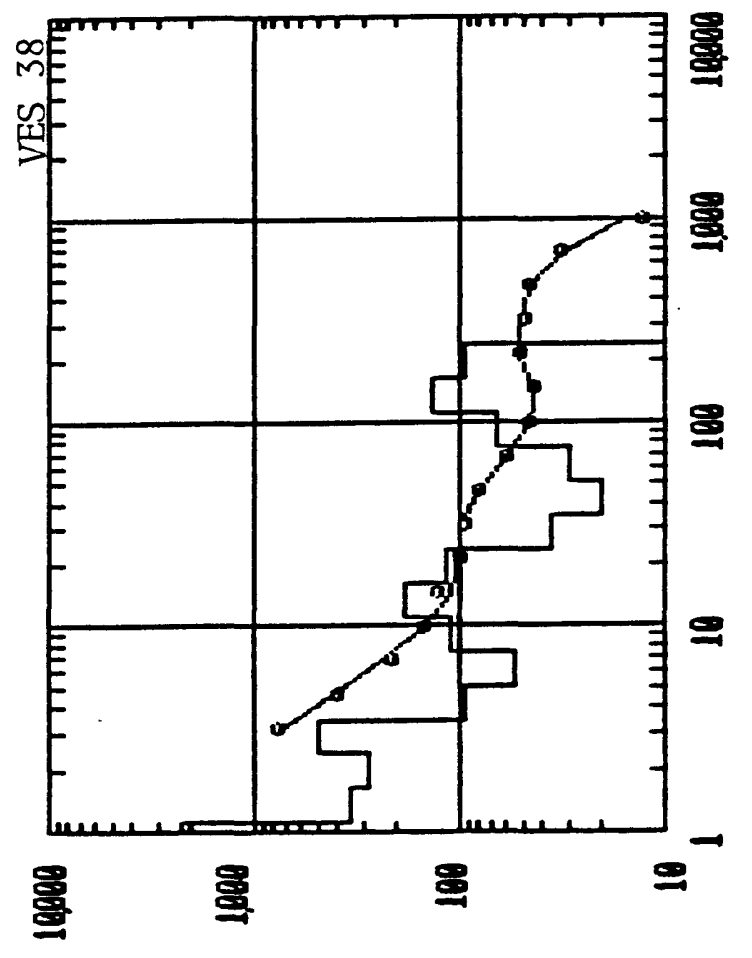

SU $\exists \perp \exists W-W H O$ NI ' $\lambda \perp I A I S I S \exists Y$
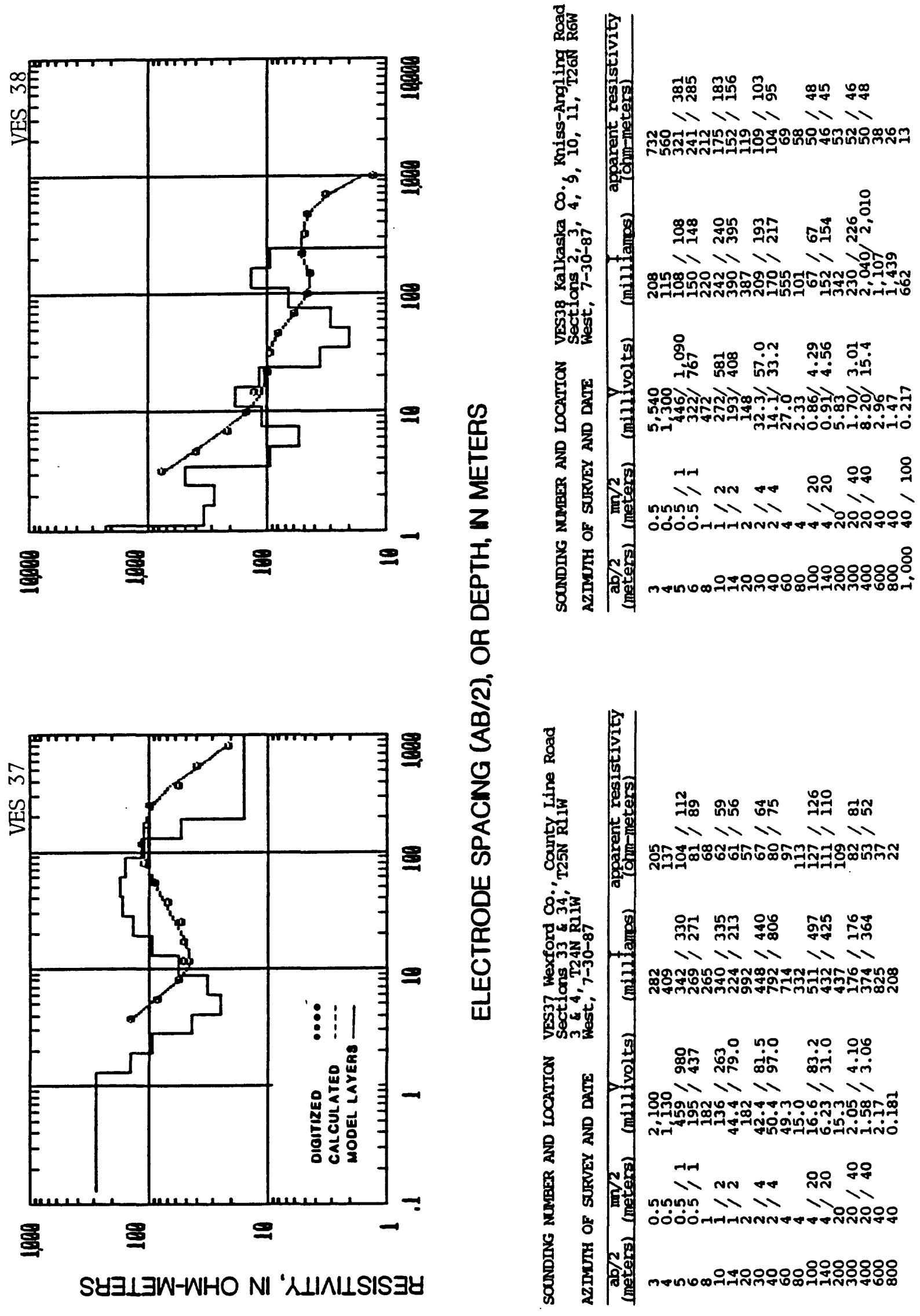

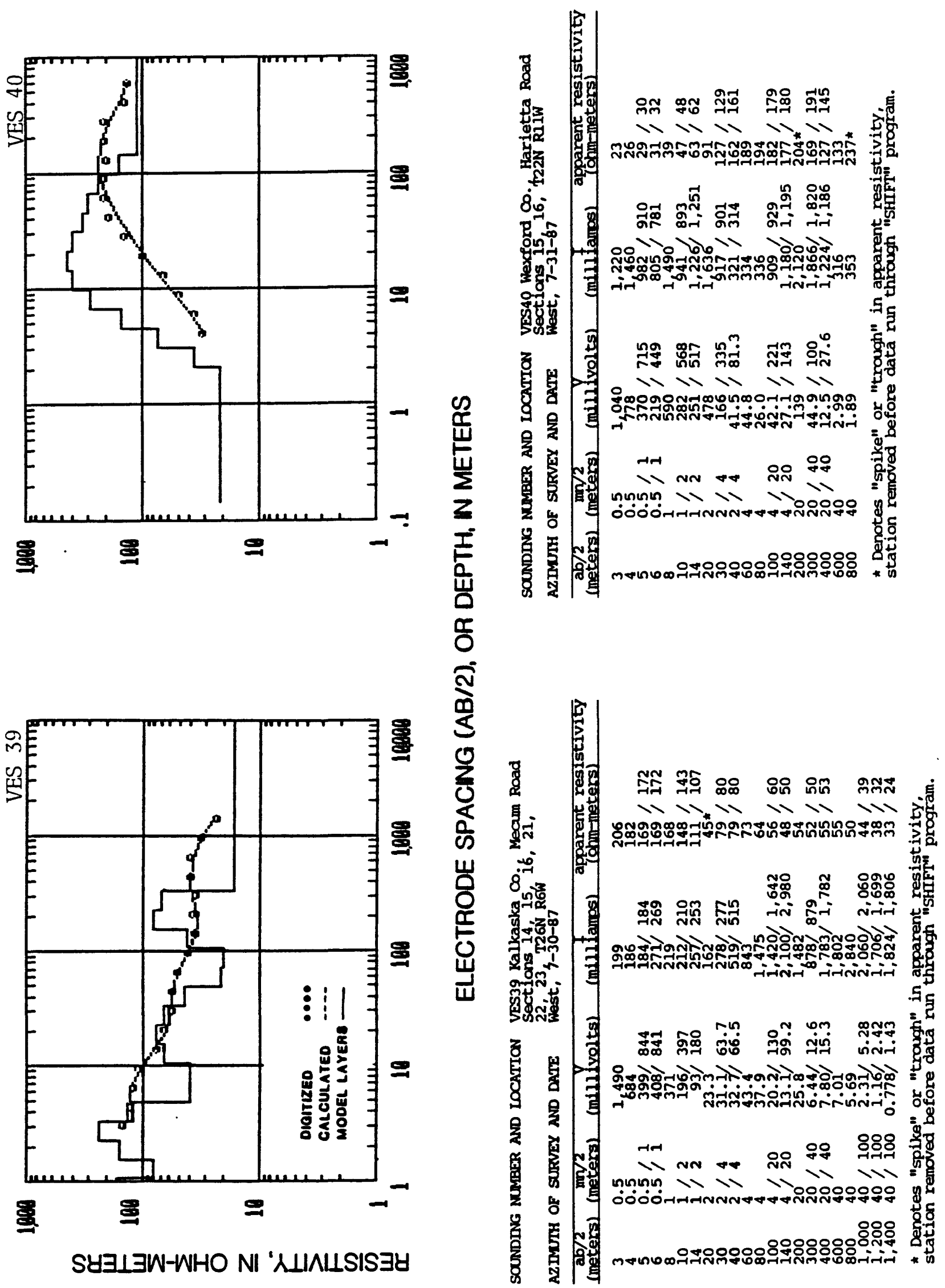

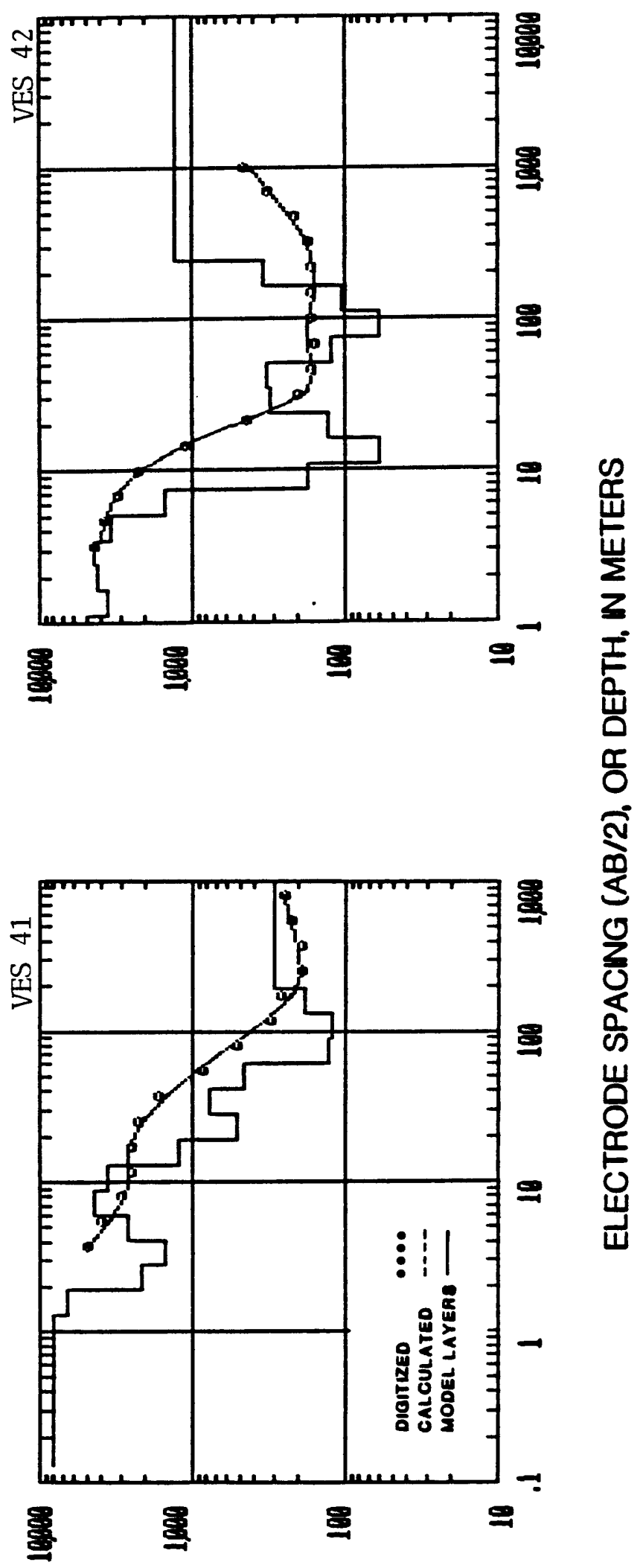

SU $\perp \exists W-W H O$ NI ' $\lambda \perp I N I S I S \exists Y$

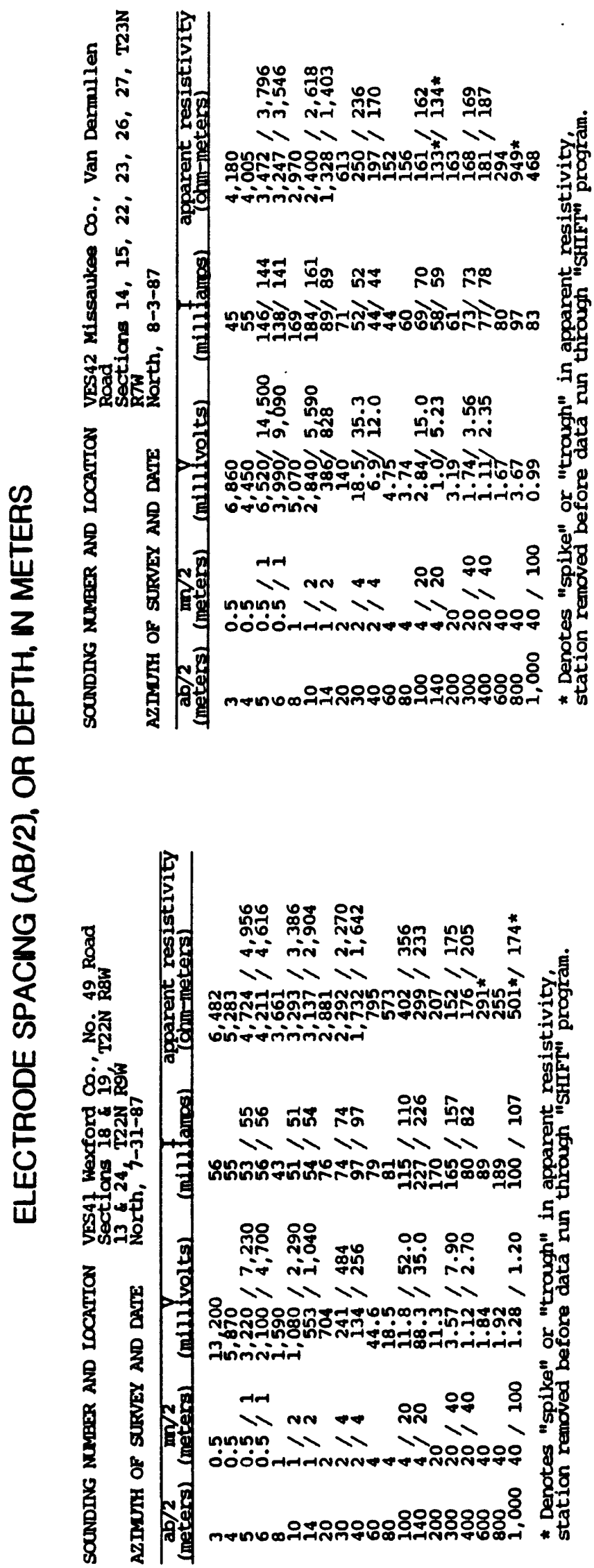



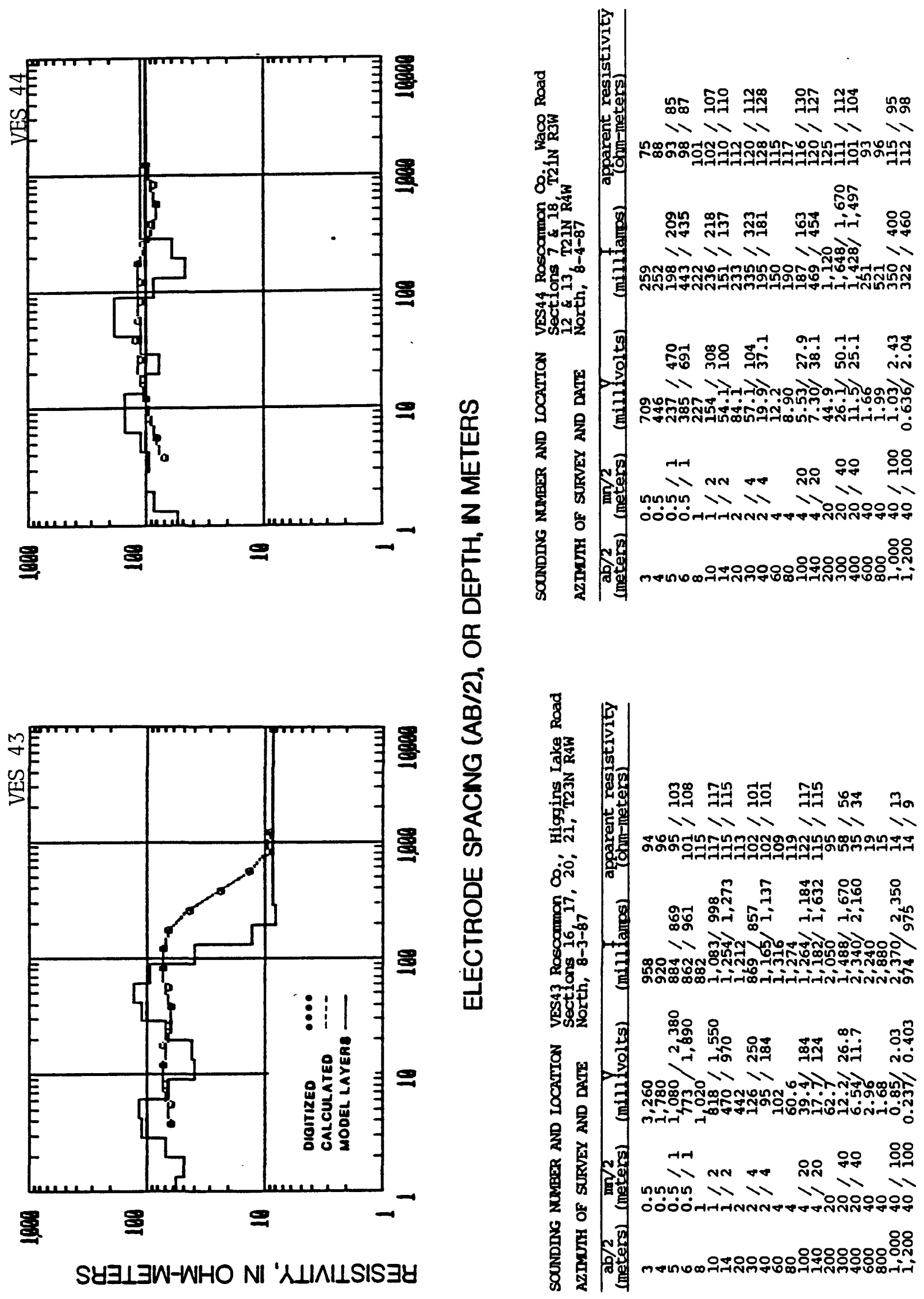

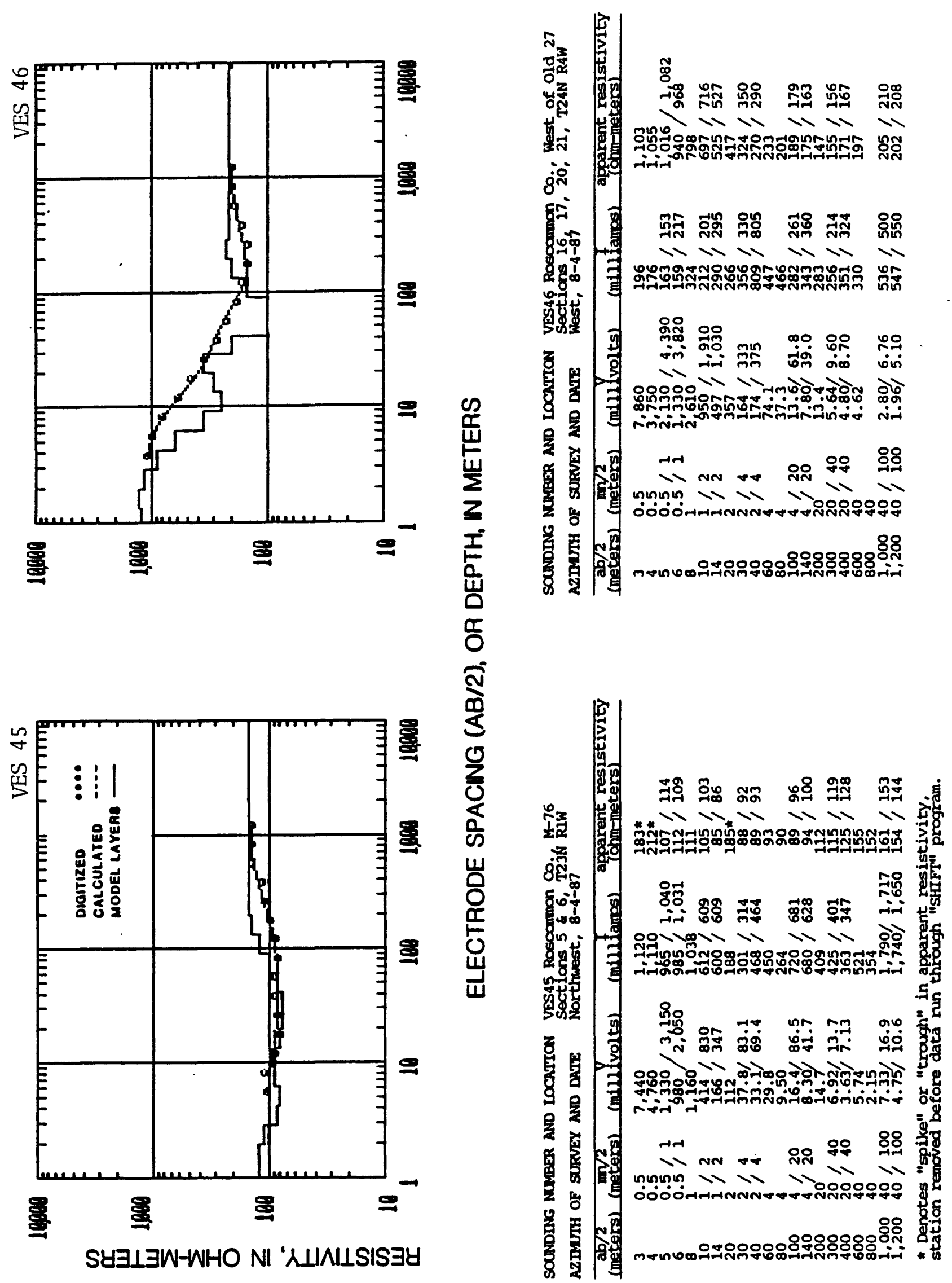

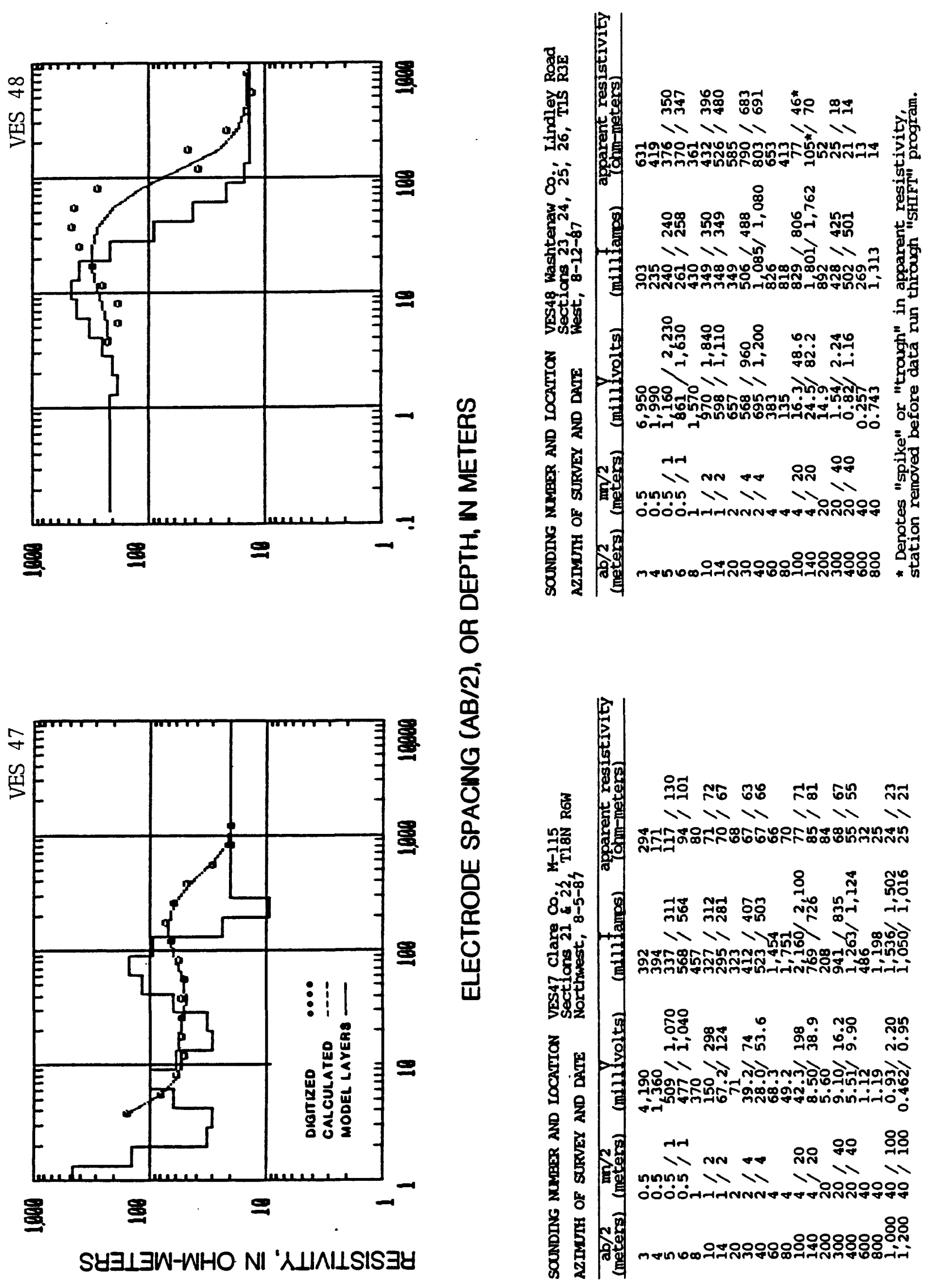

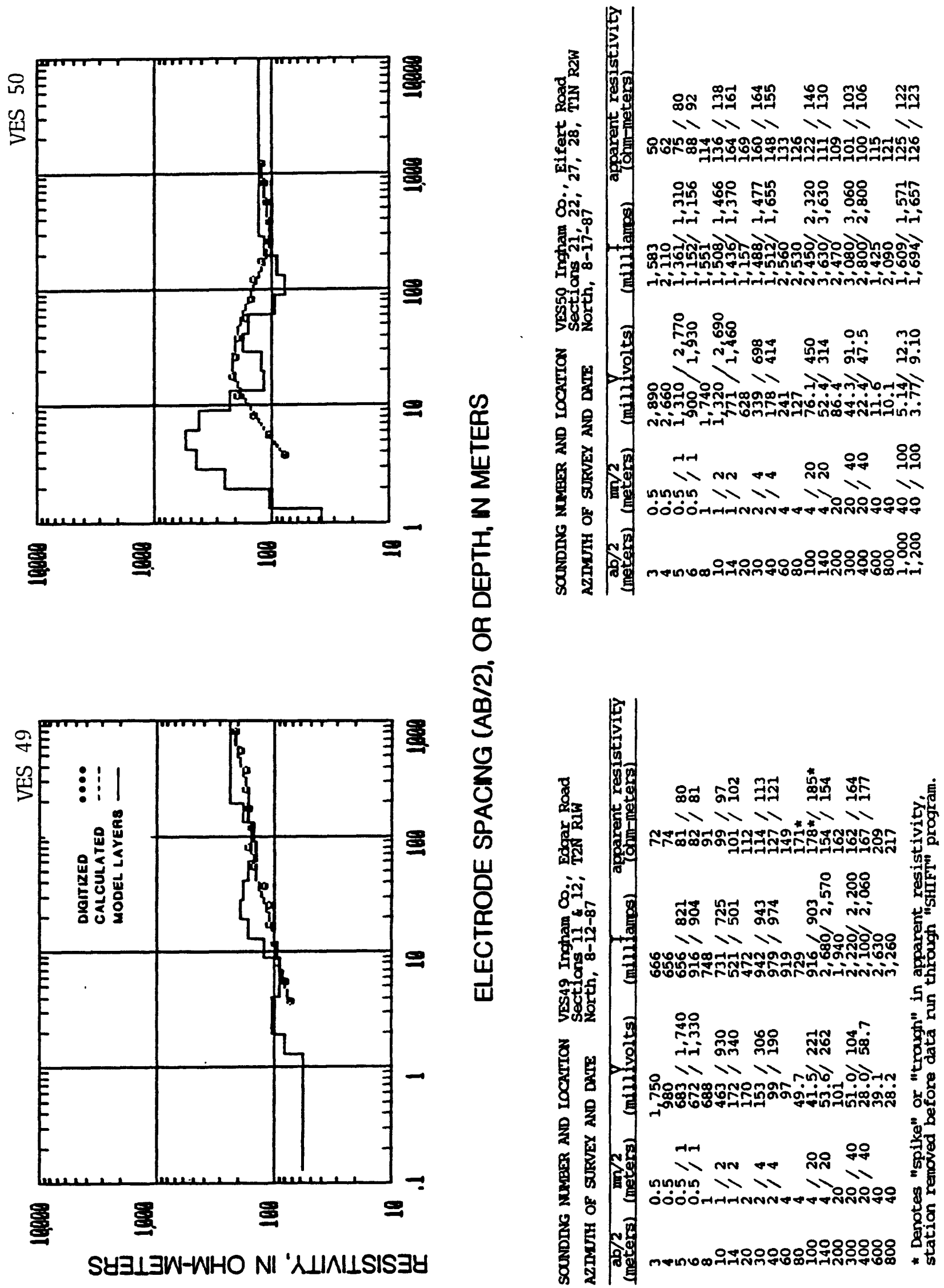

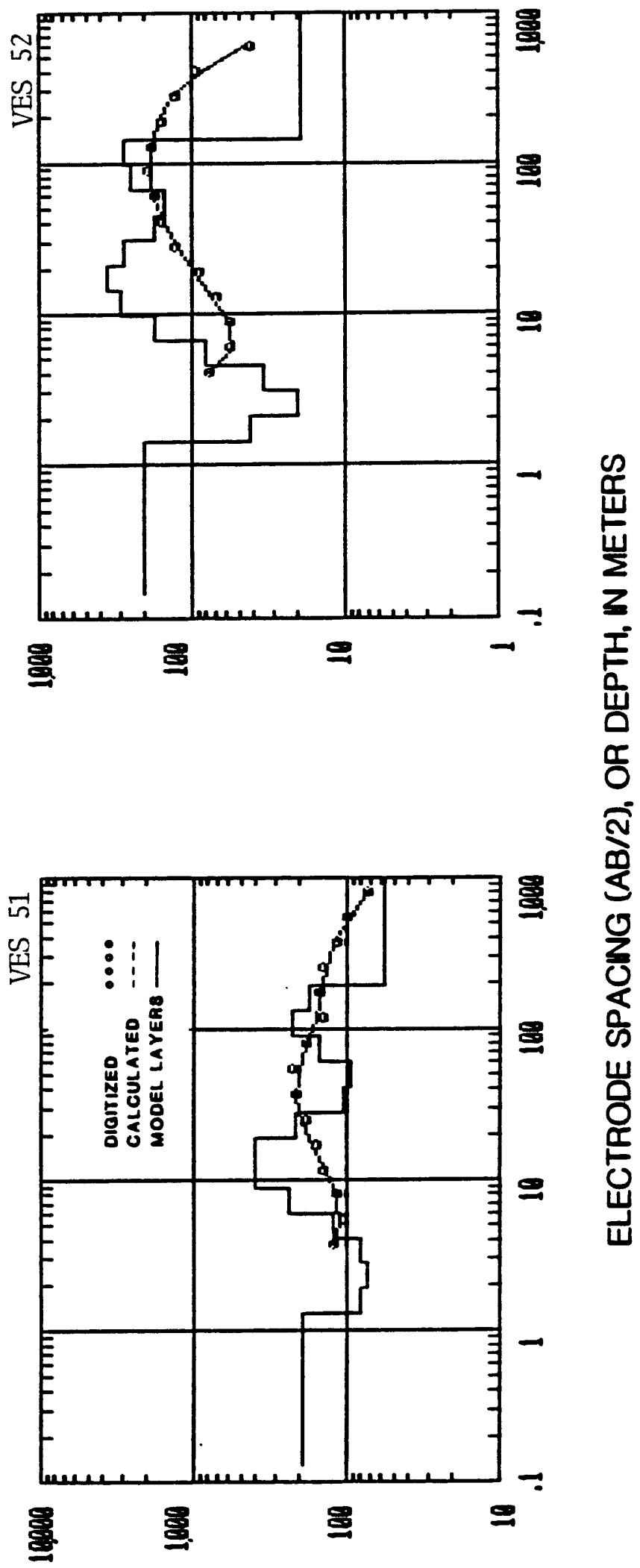

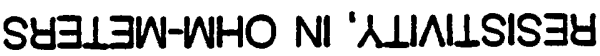

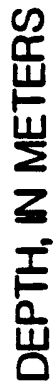

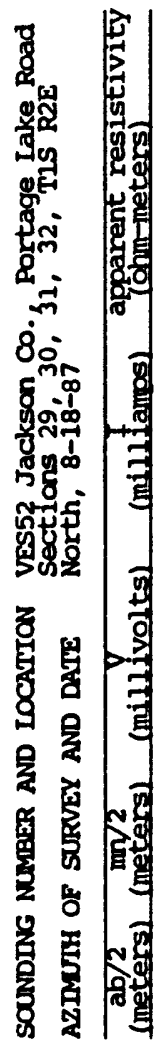

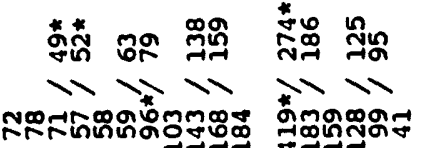

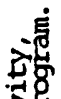

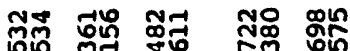

N N W N

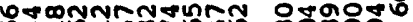

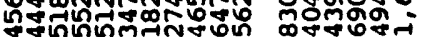

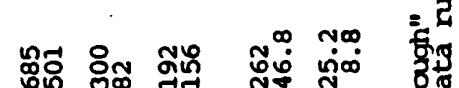

네 W W

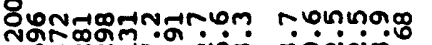

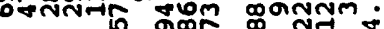

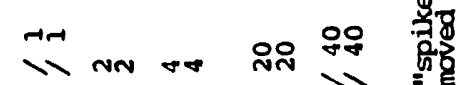

nnunn W N W

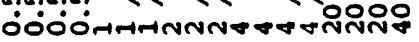

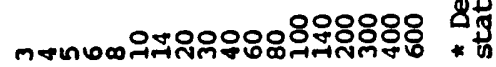

号

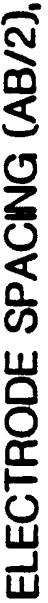

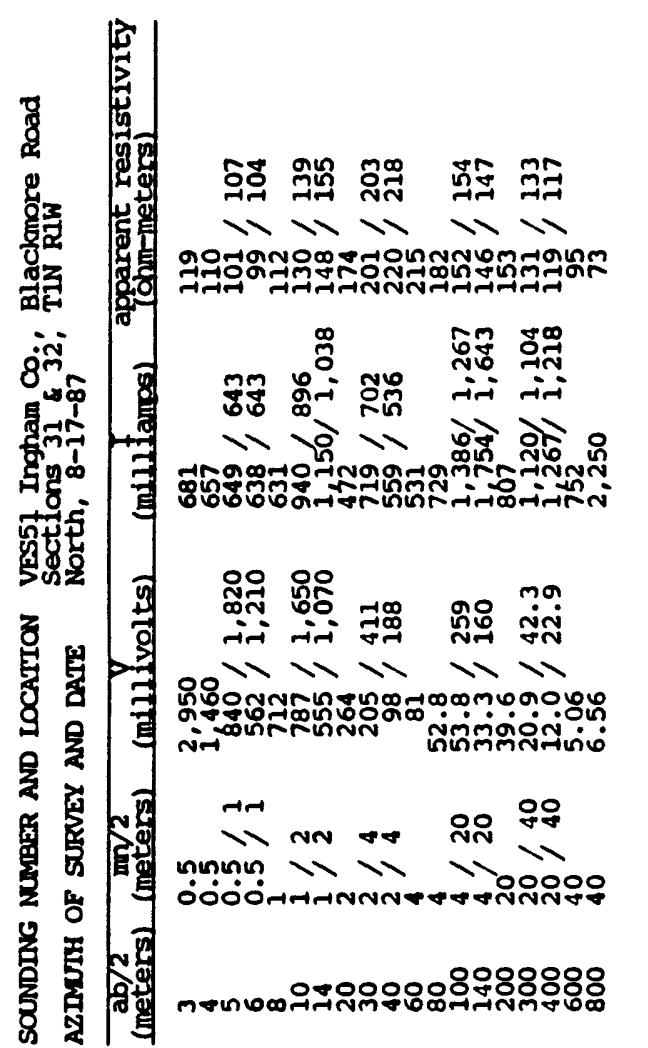



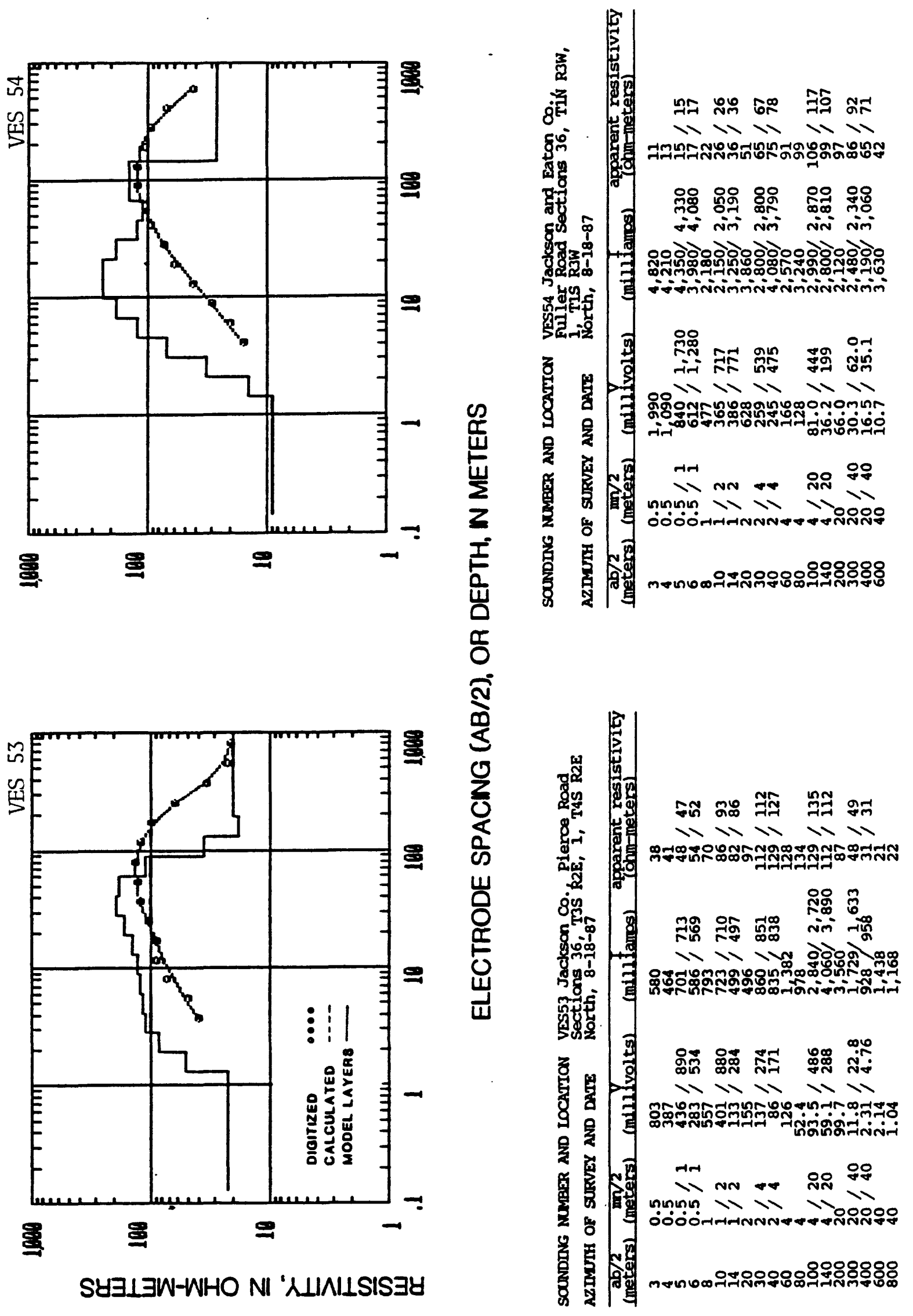


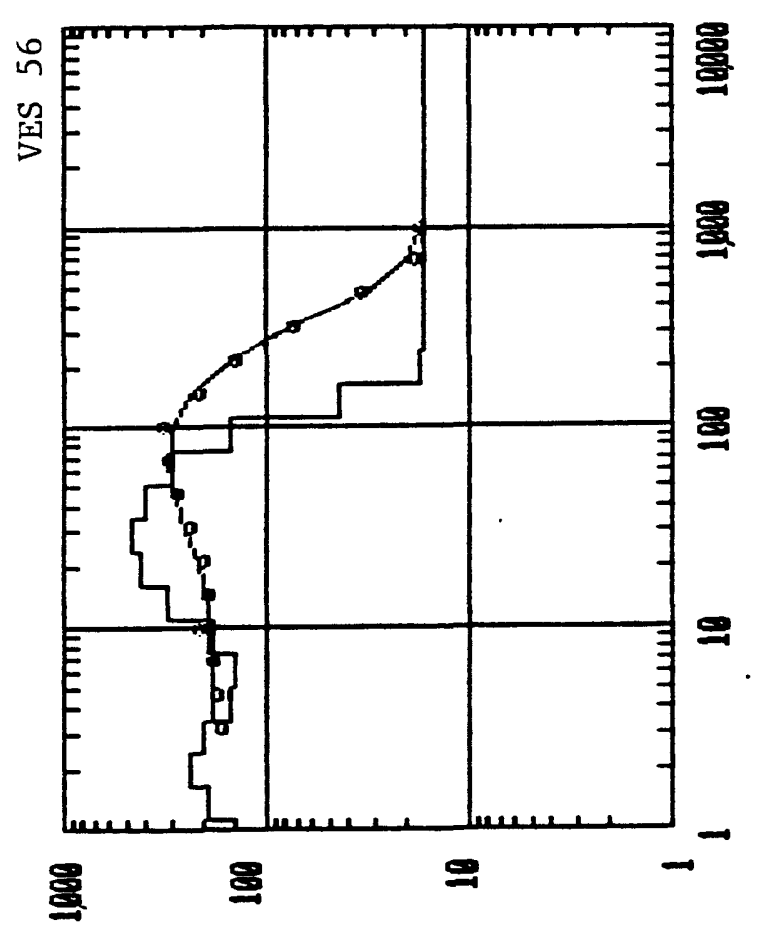

里

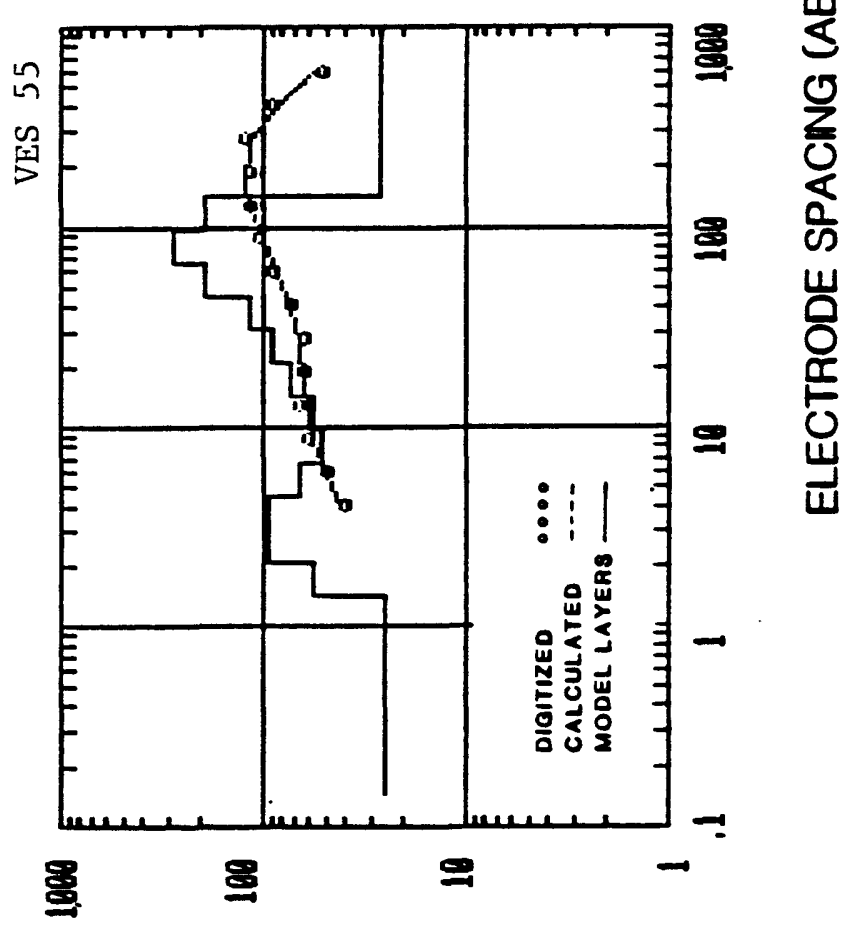

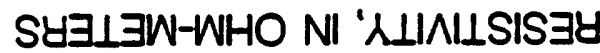

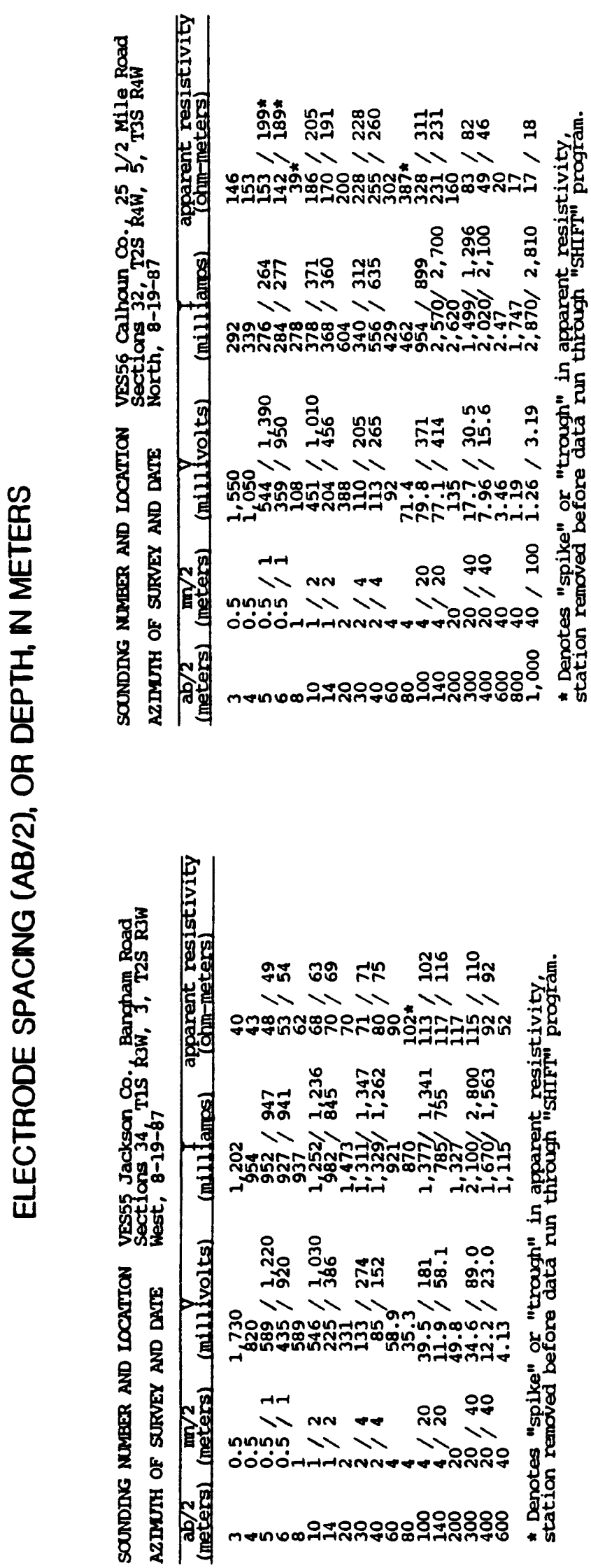



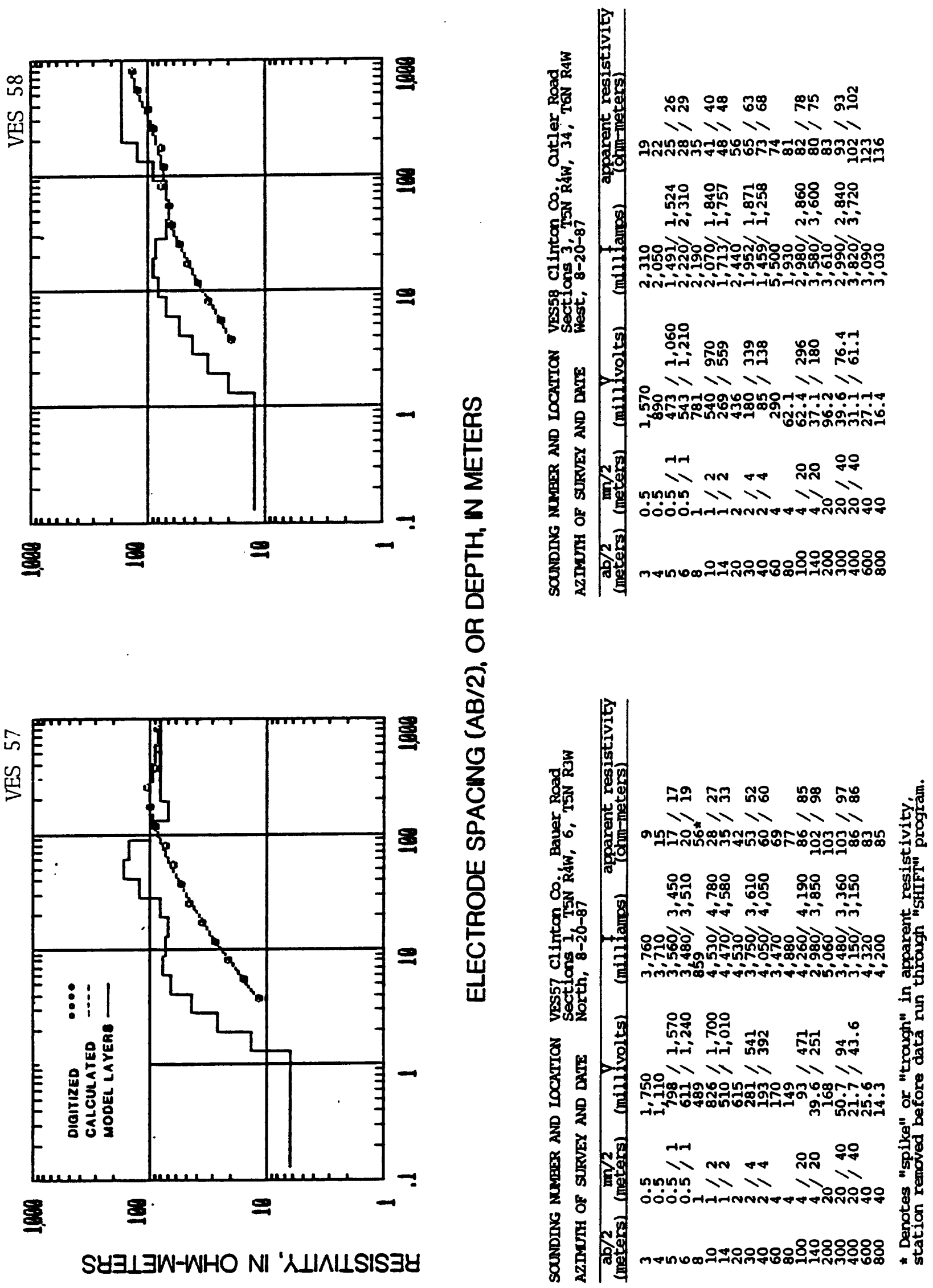

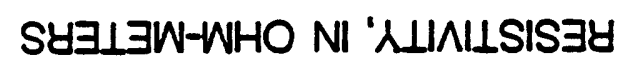

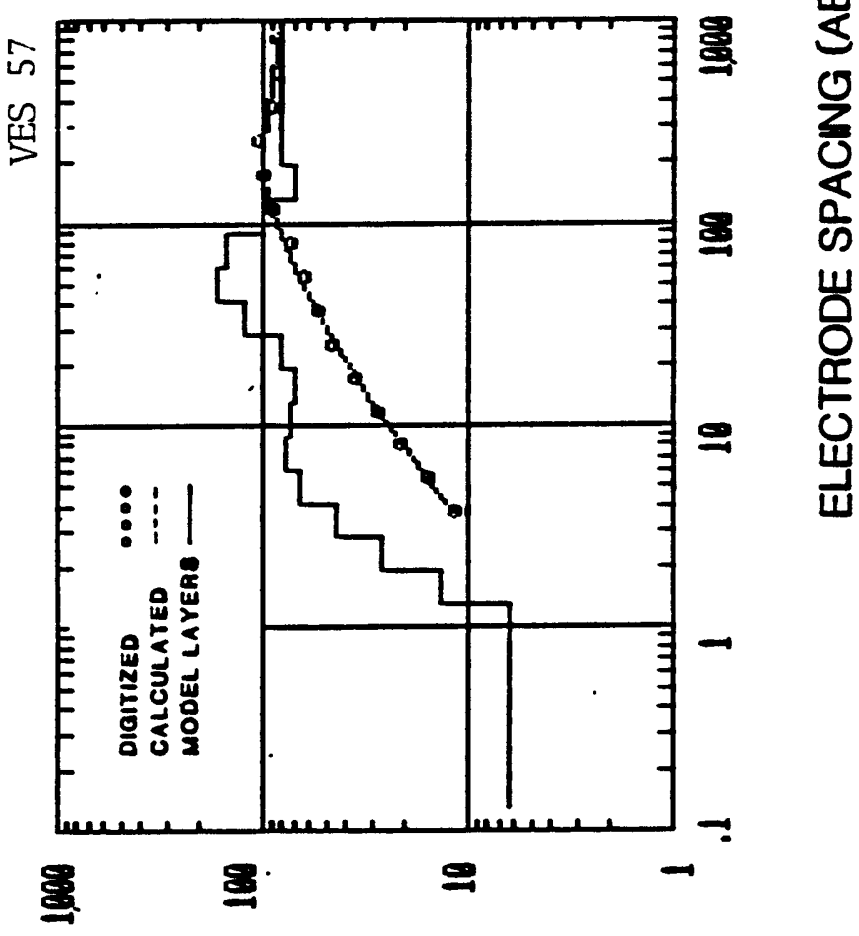

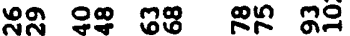

认 N

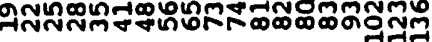

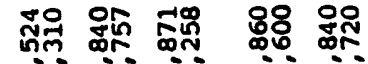

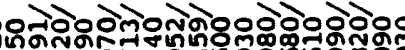

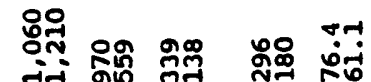

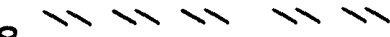

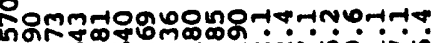

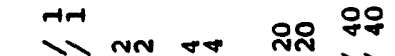

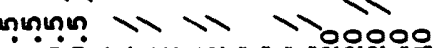
m

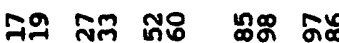

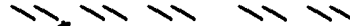

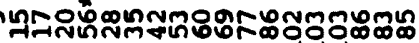

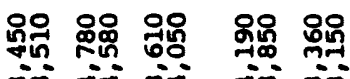

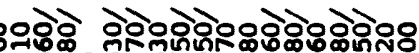
ก!

웅용우

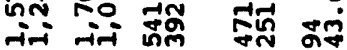
ר) W W W

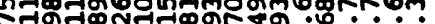
तn-

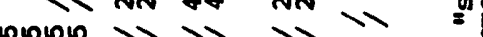
๑000-1 

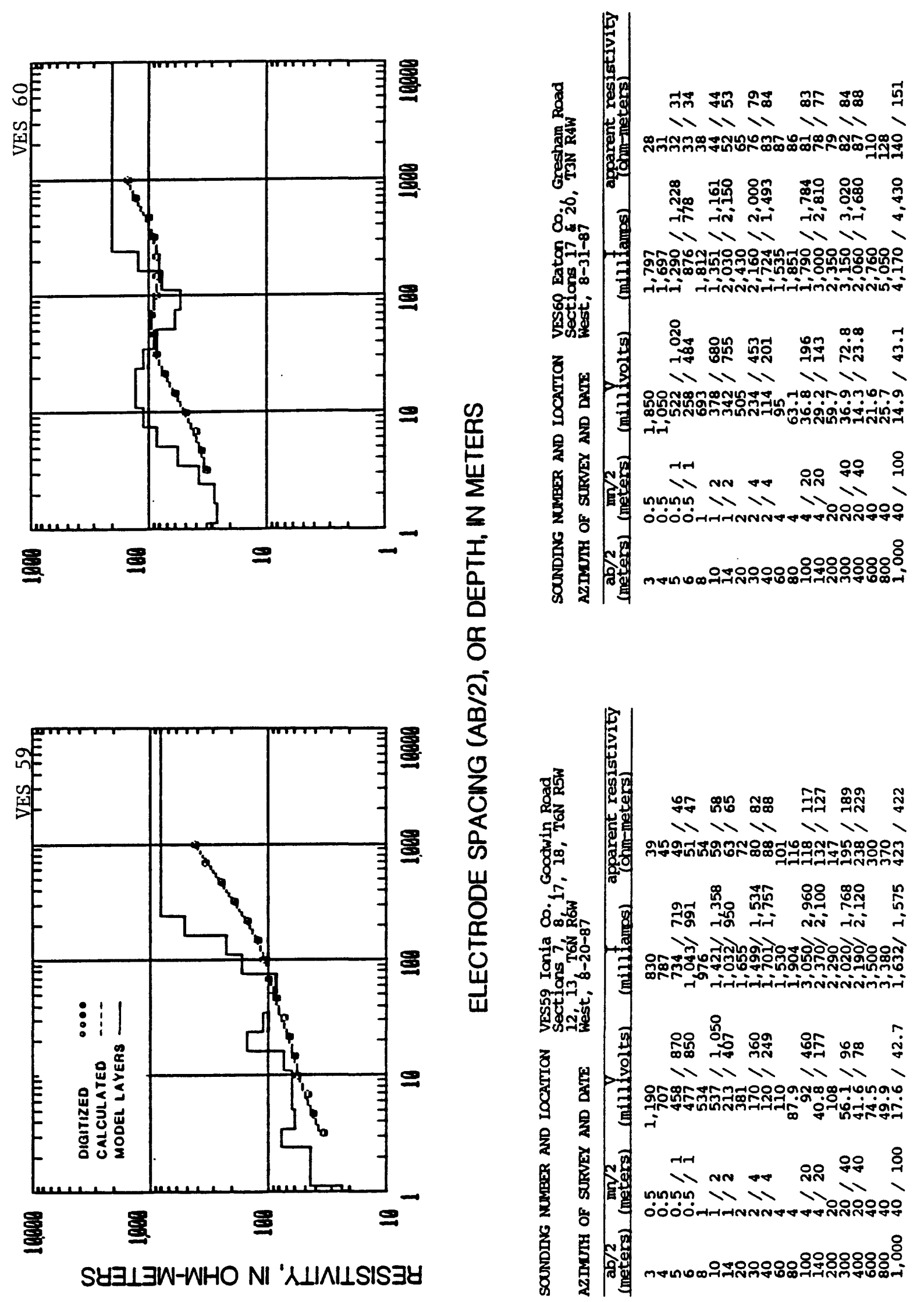

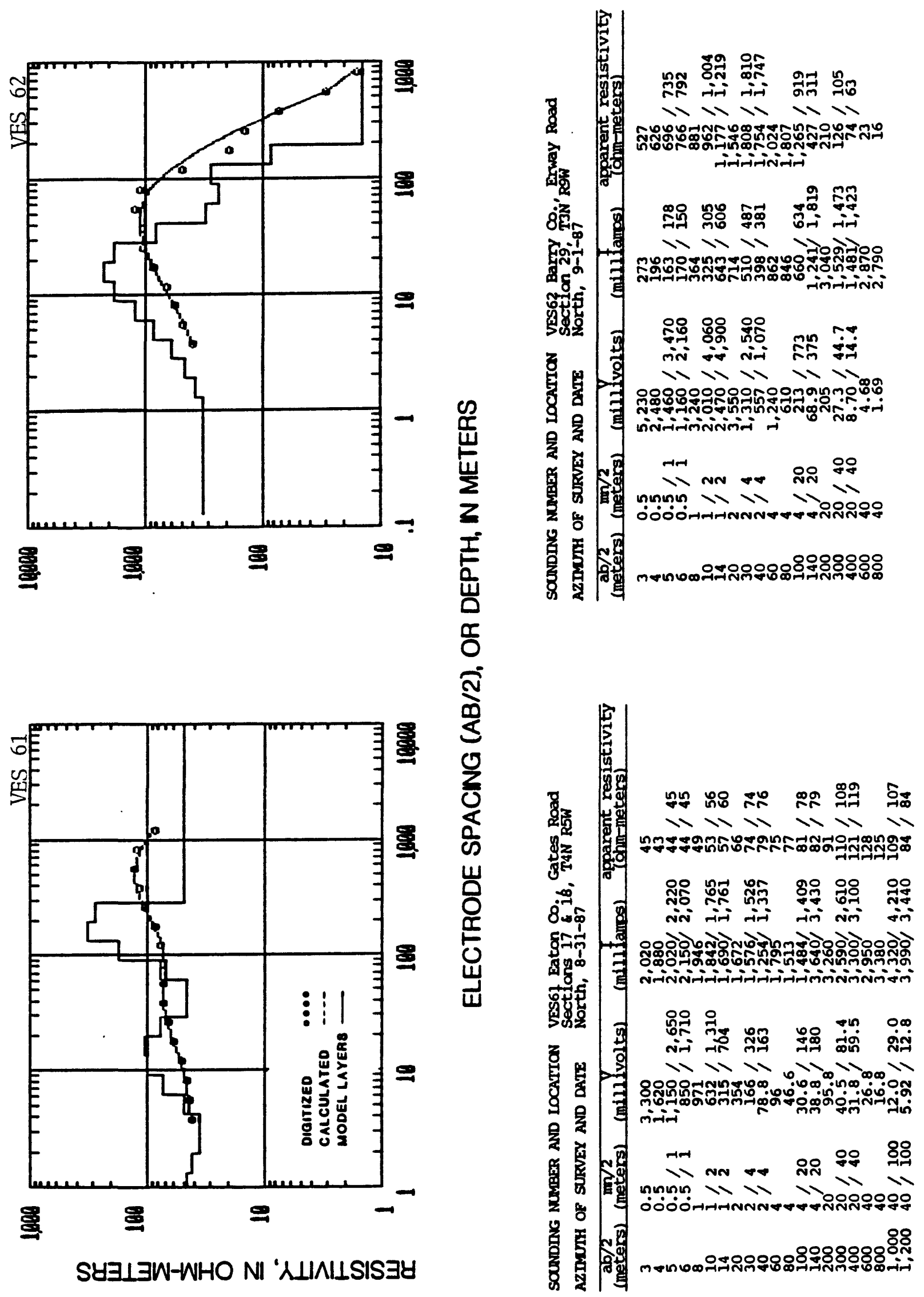

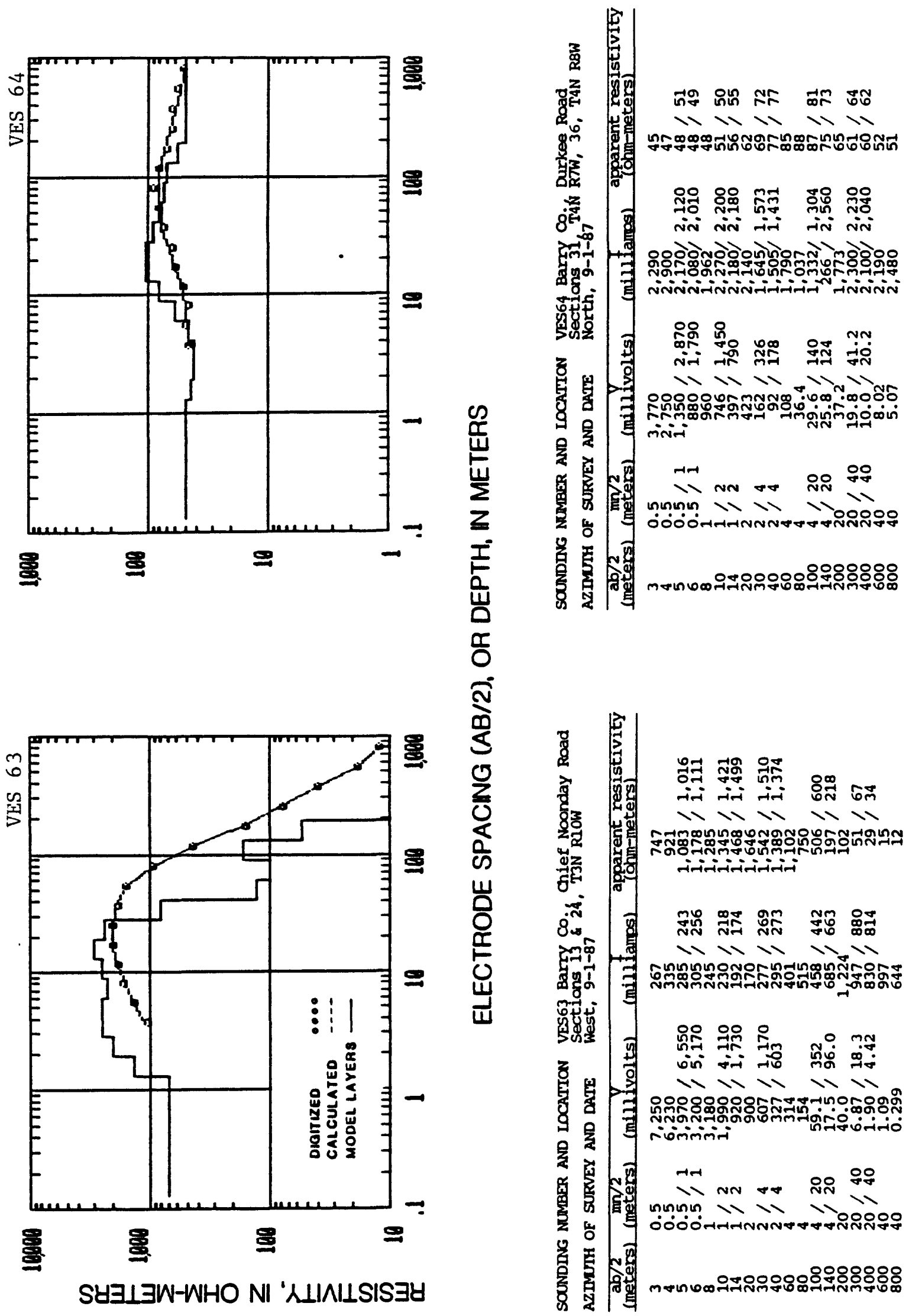

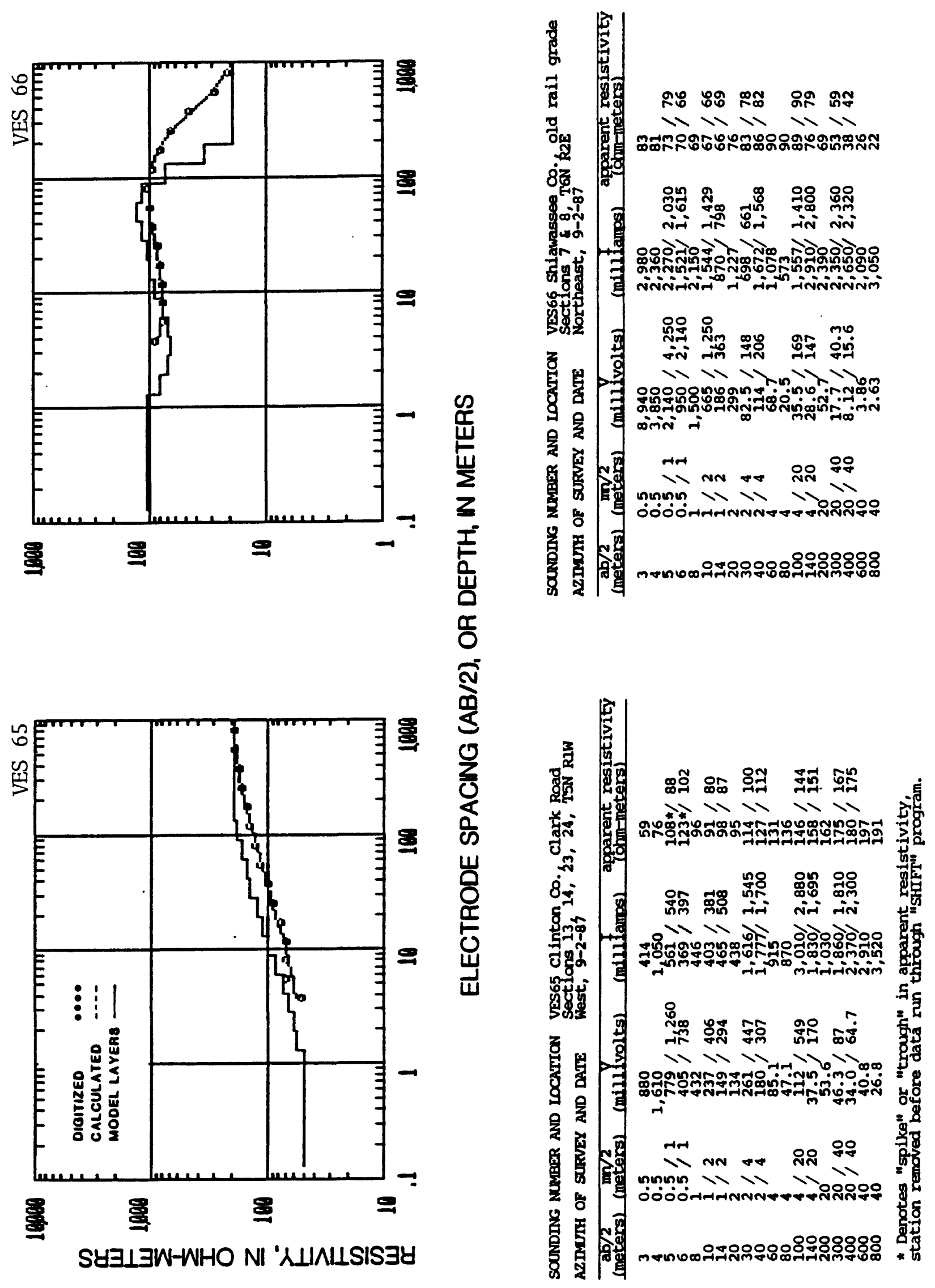

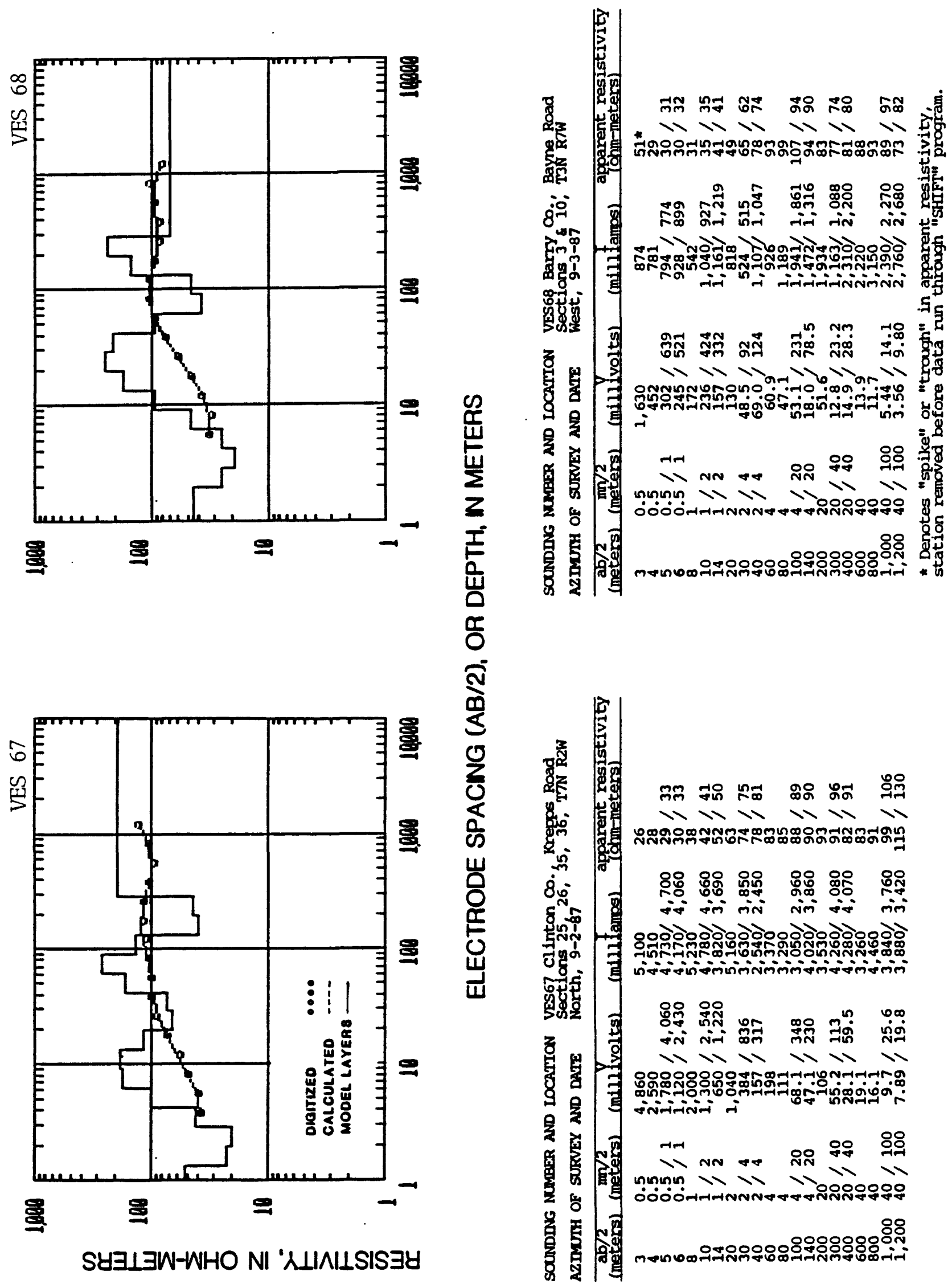

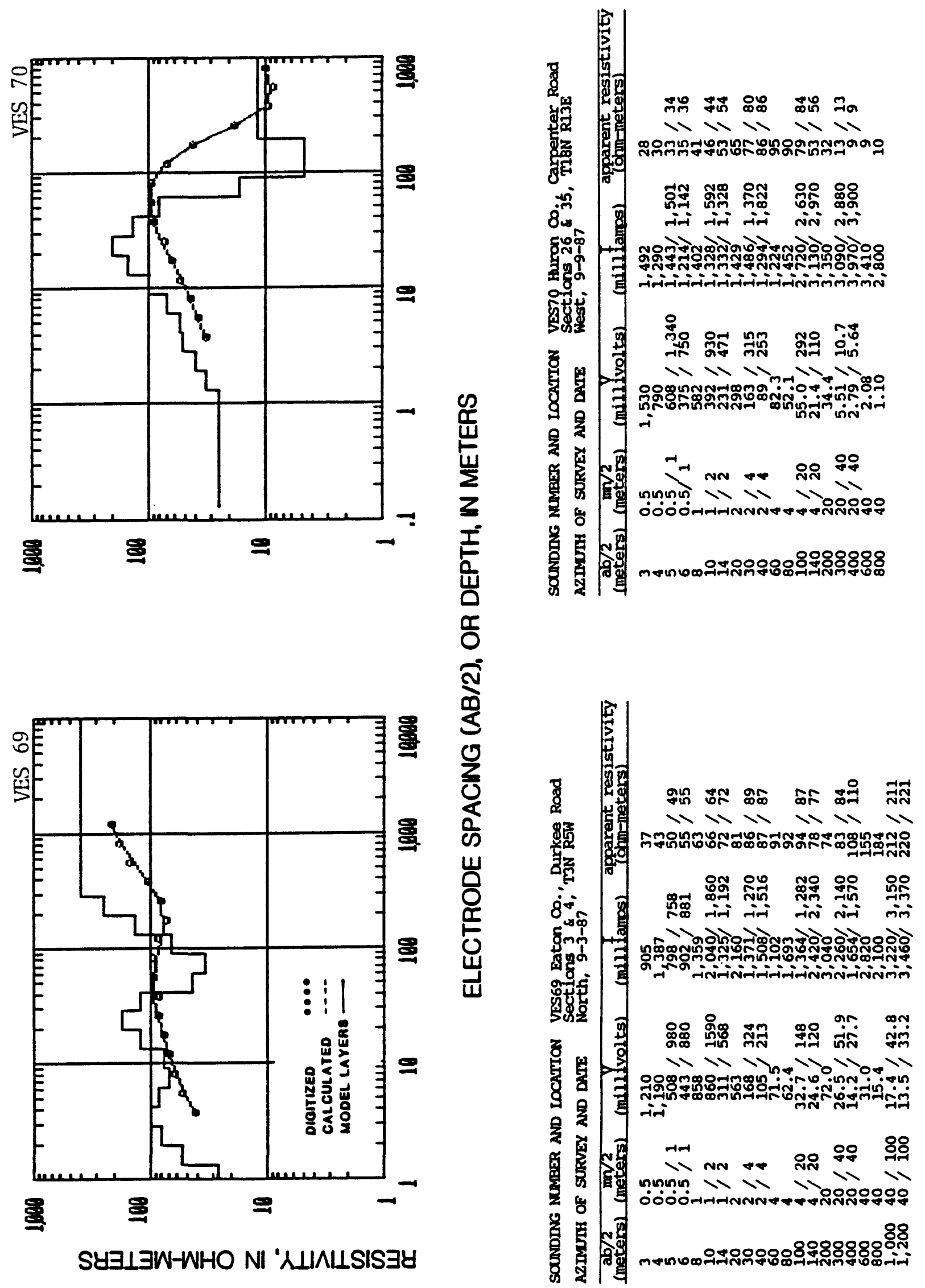

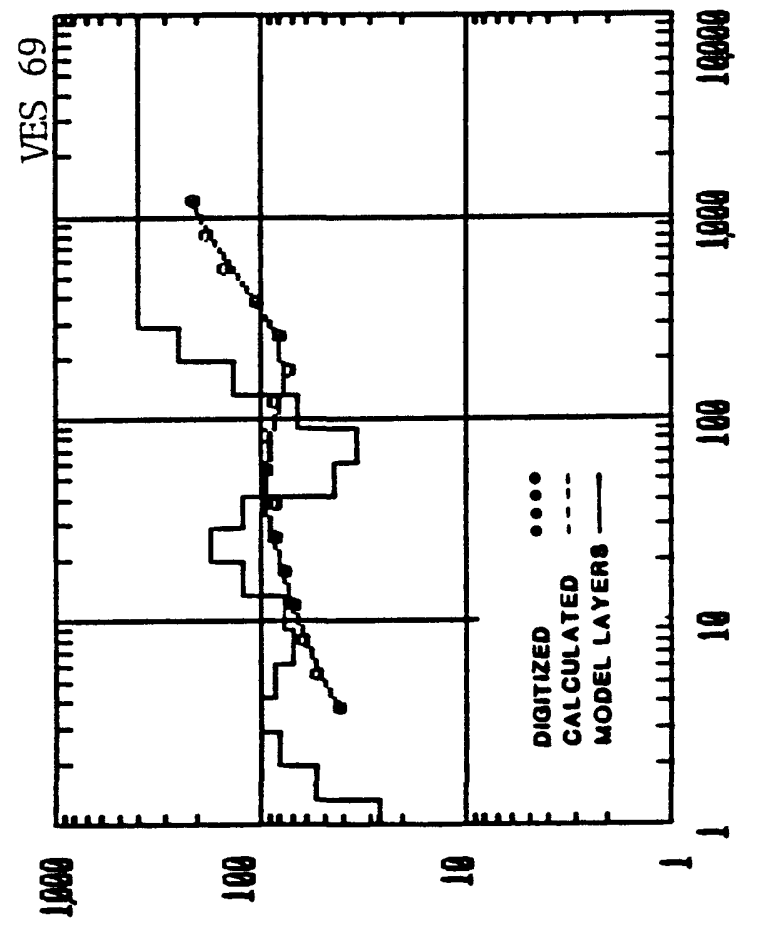

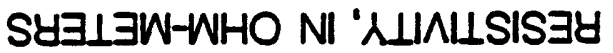

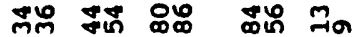

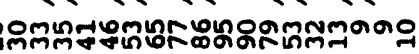

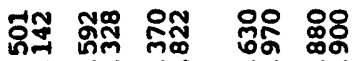
नifi rin in viv nim

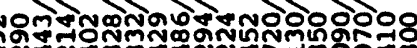

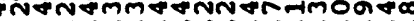

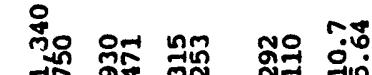

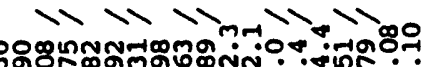
คิㅇำ $\mathrm{N}^{-1}$ nn +o nnino? V V t mเుn

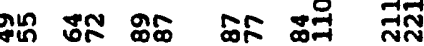

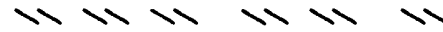
คิ

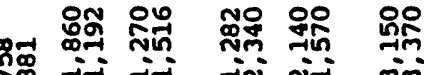
దิ on-in-in-i-in-inimininimm

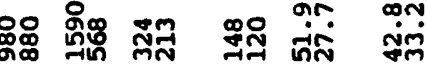

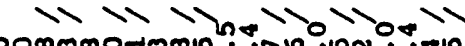

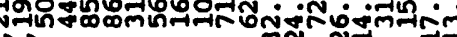
개 $\mathrm{nn}$ +4

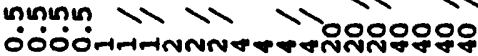

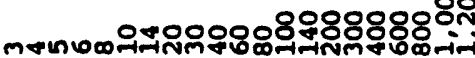



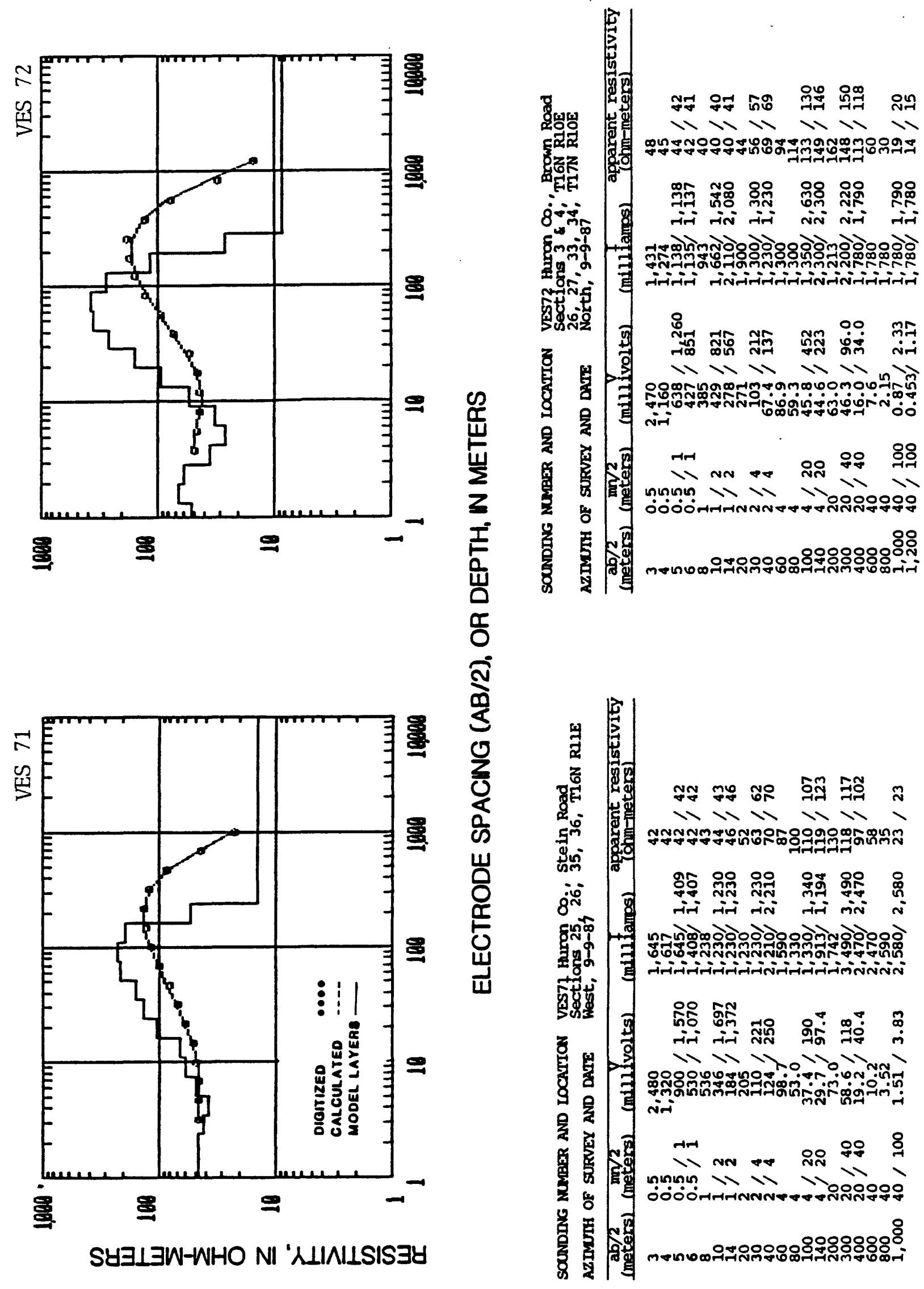

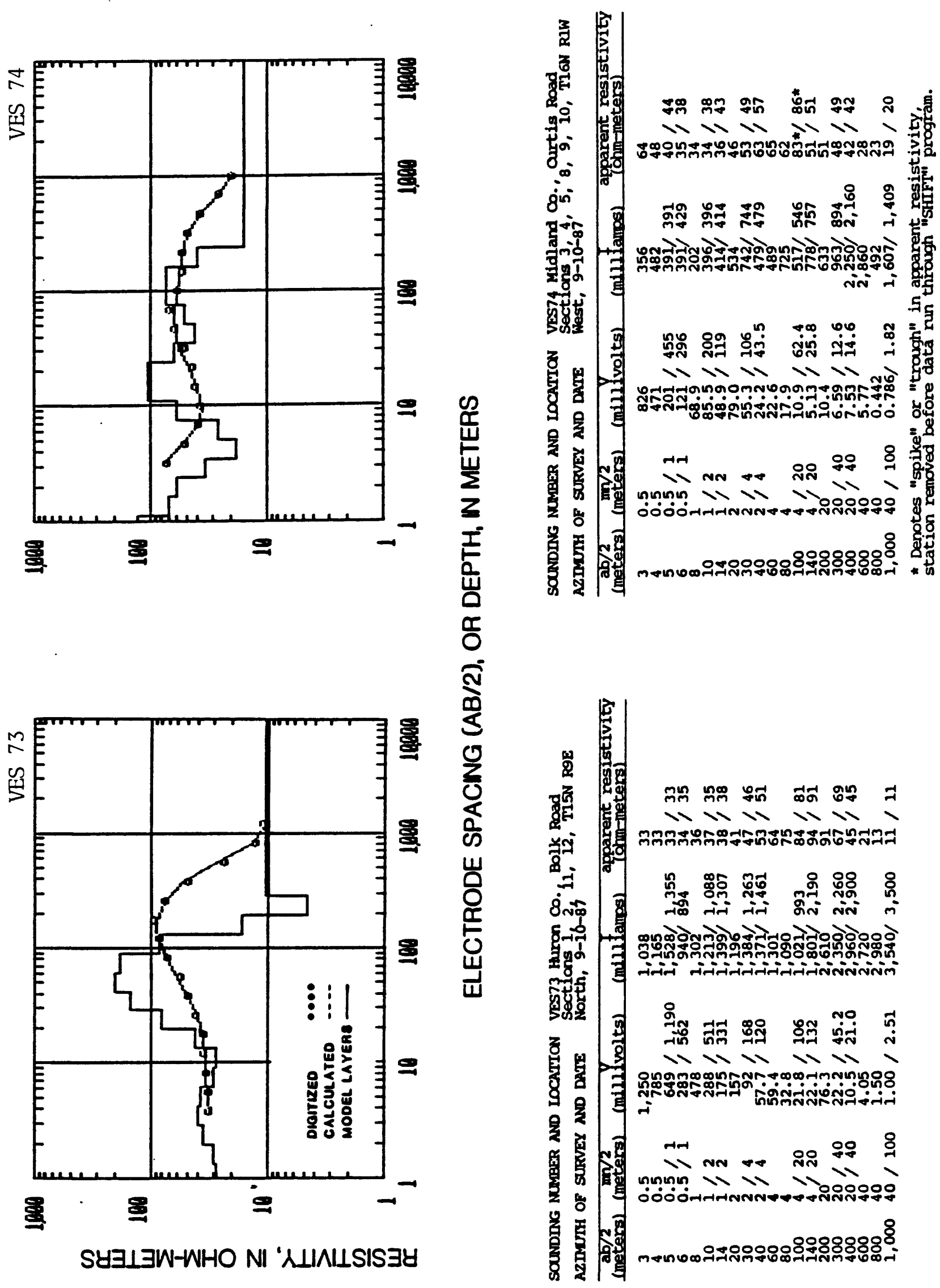

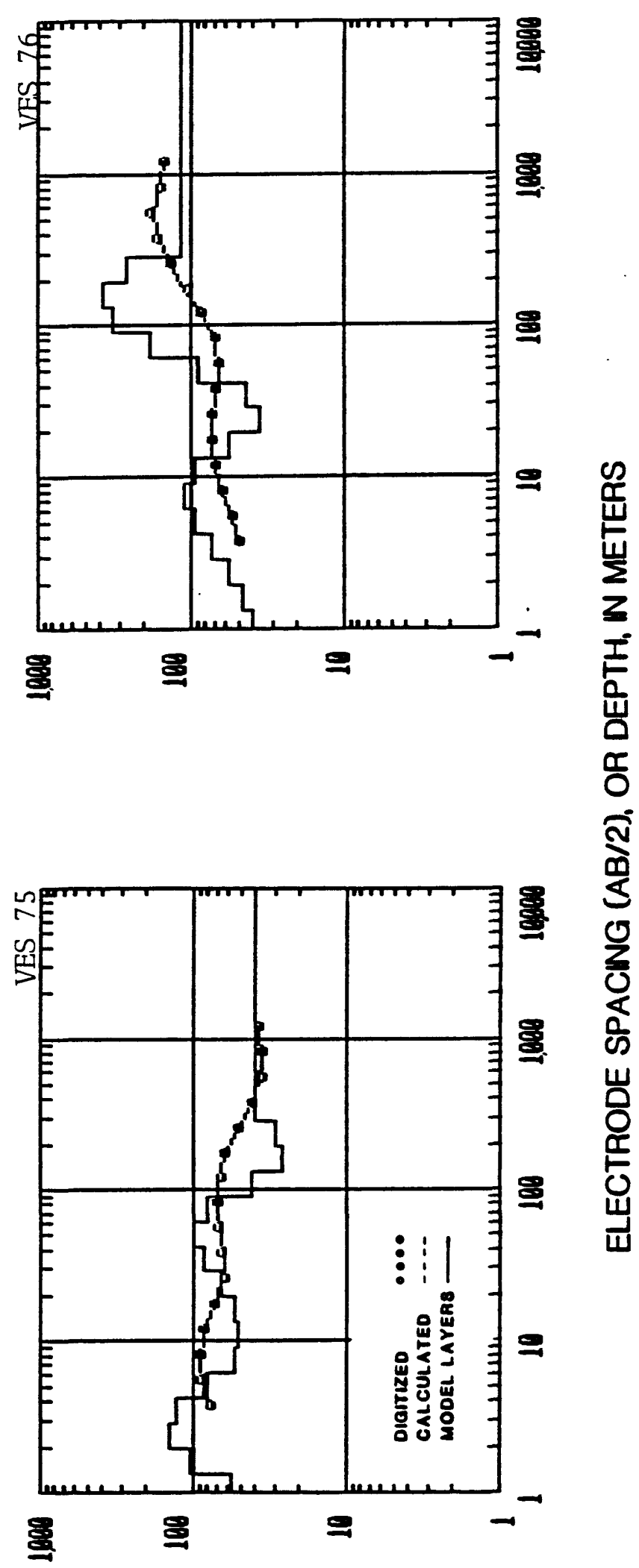

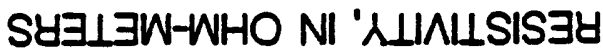

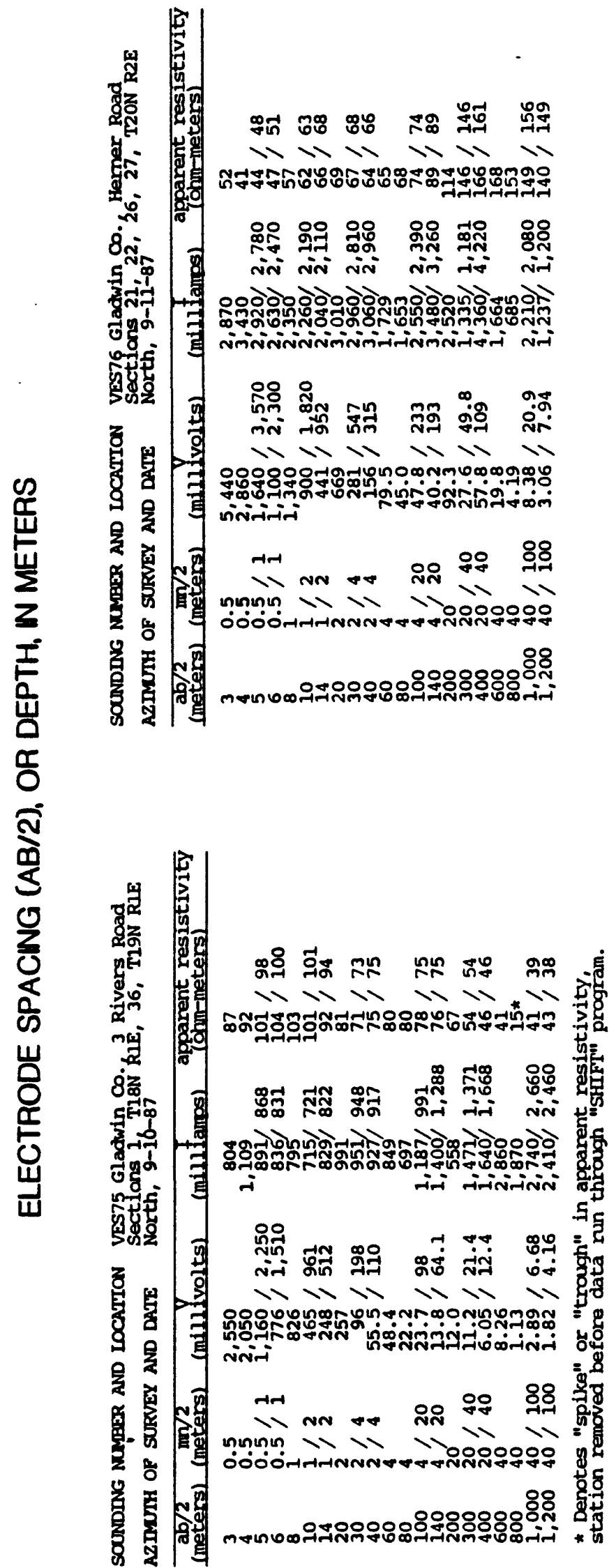



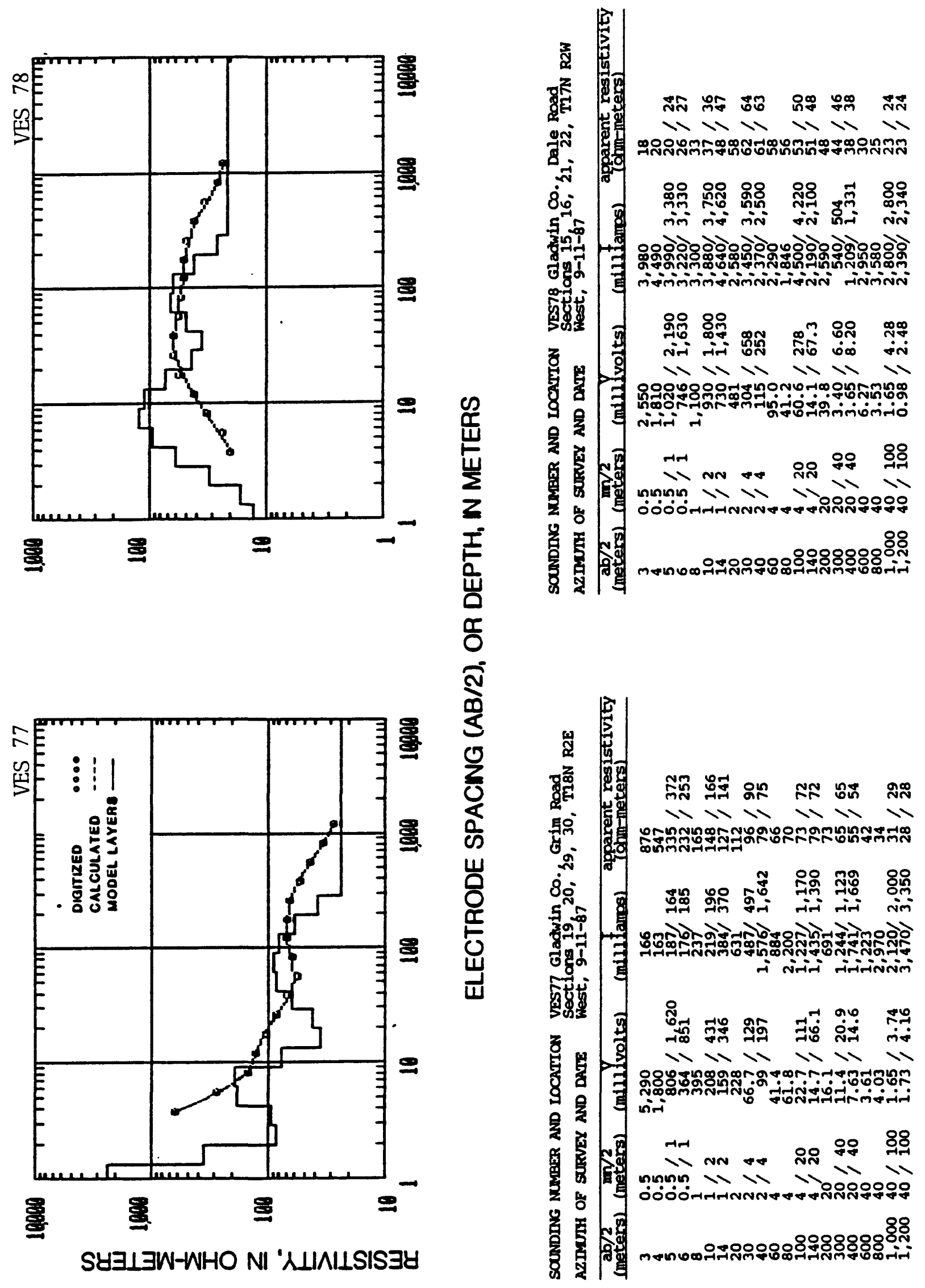

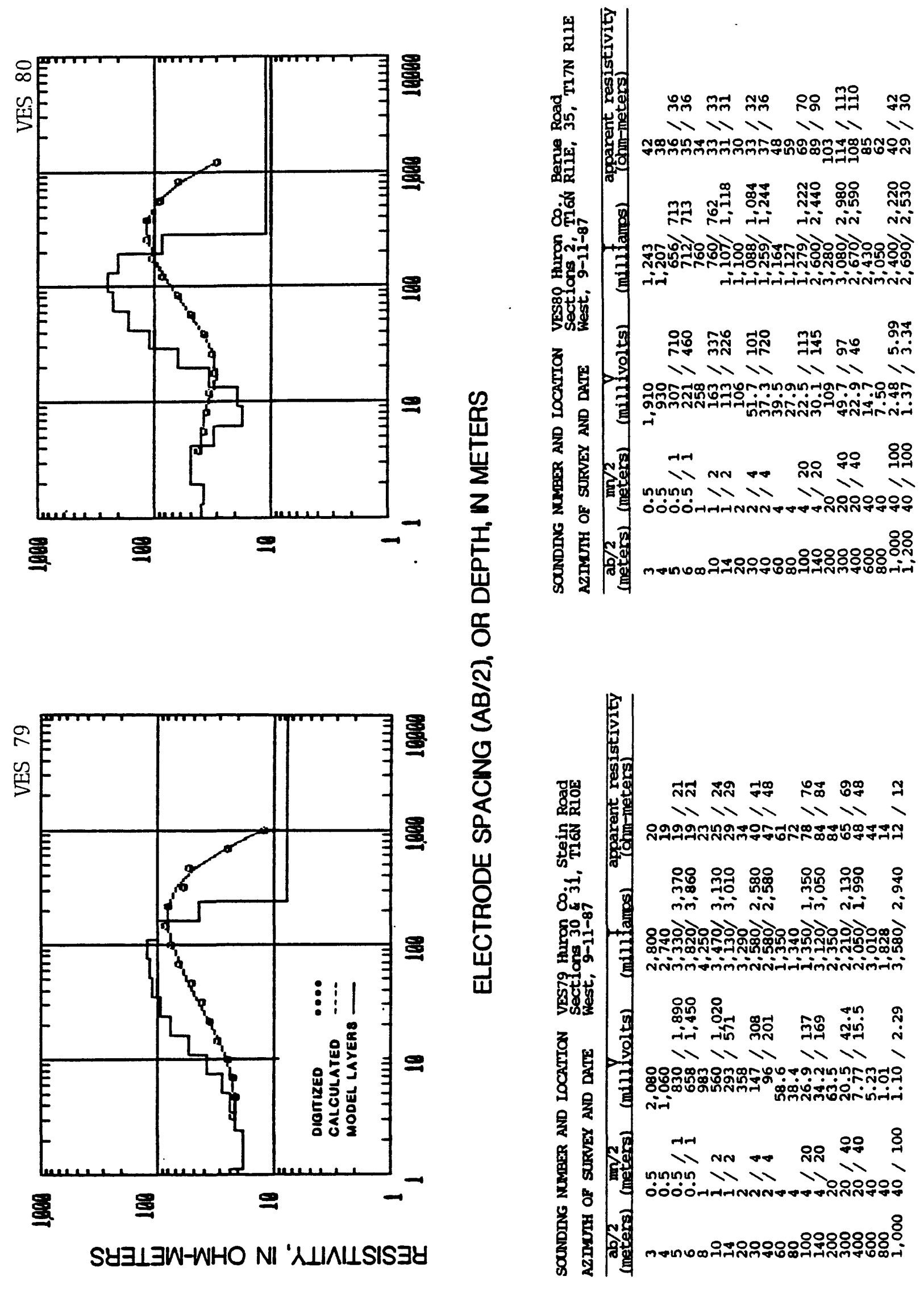

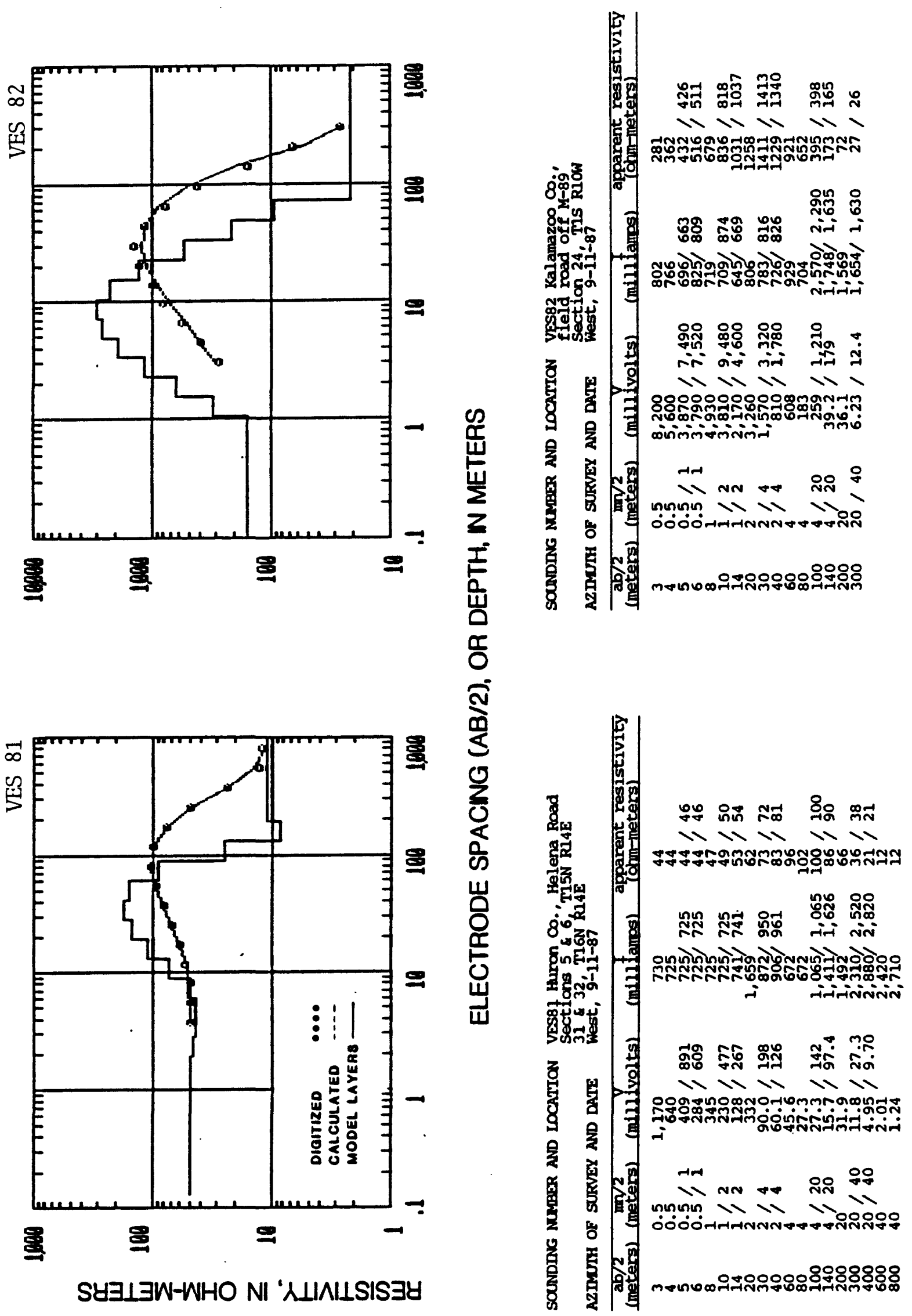


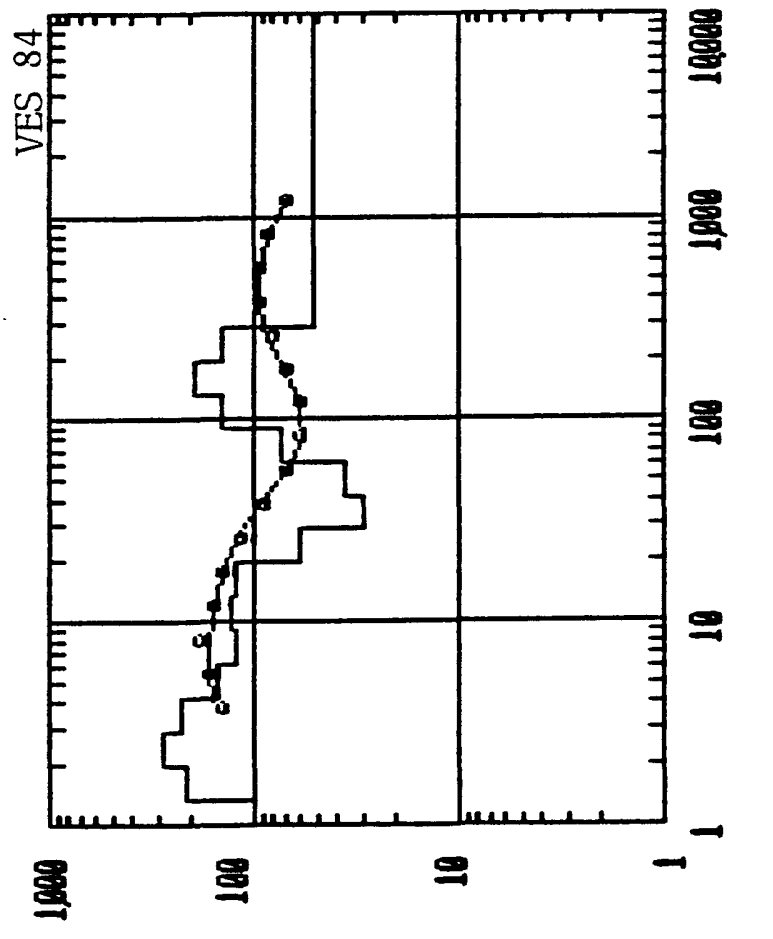

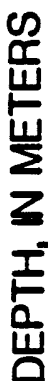

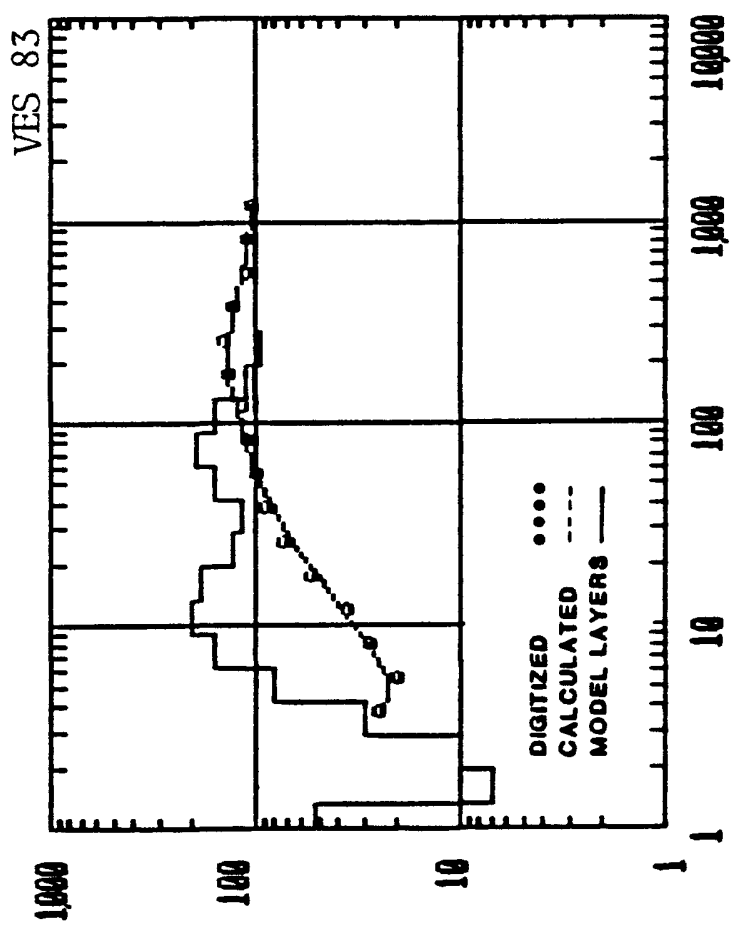

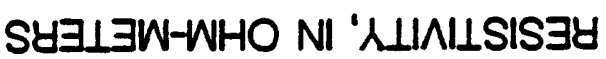

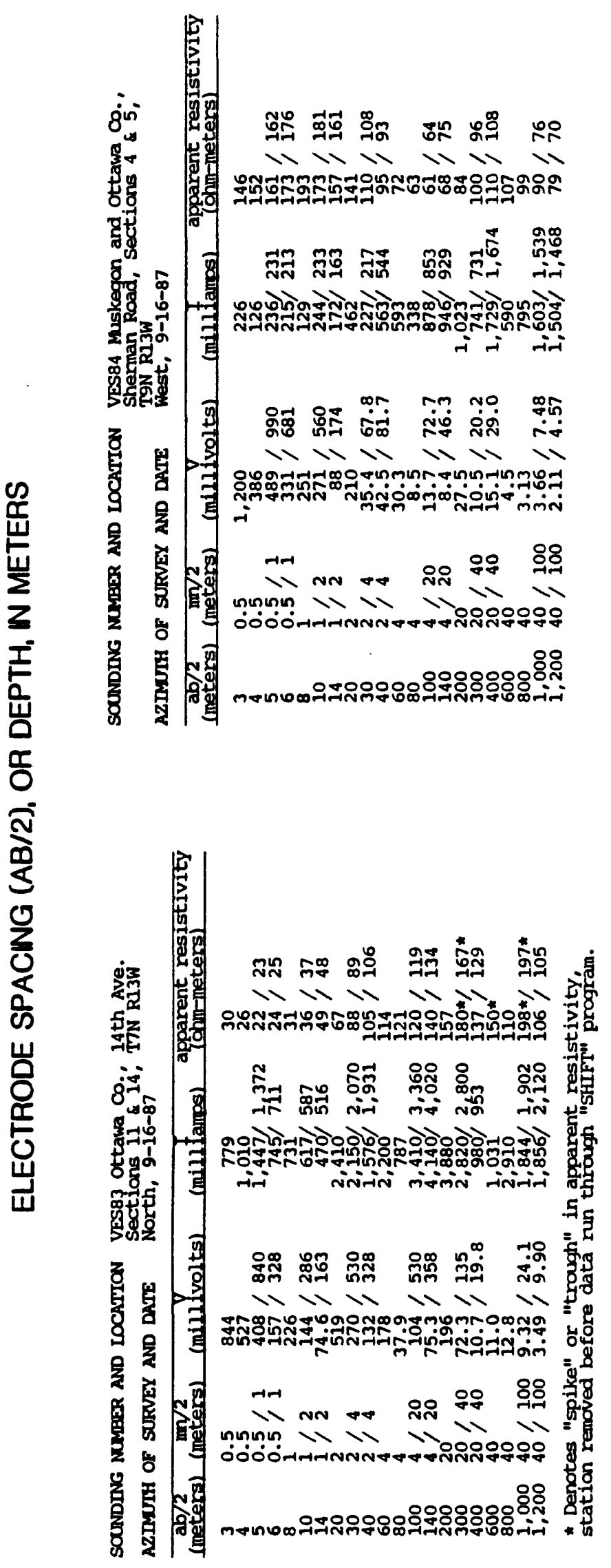



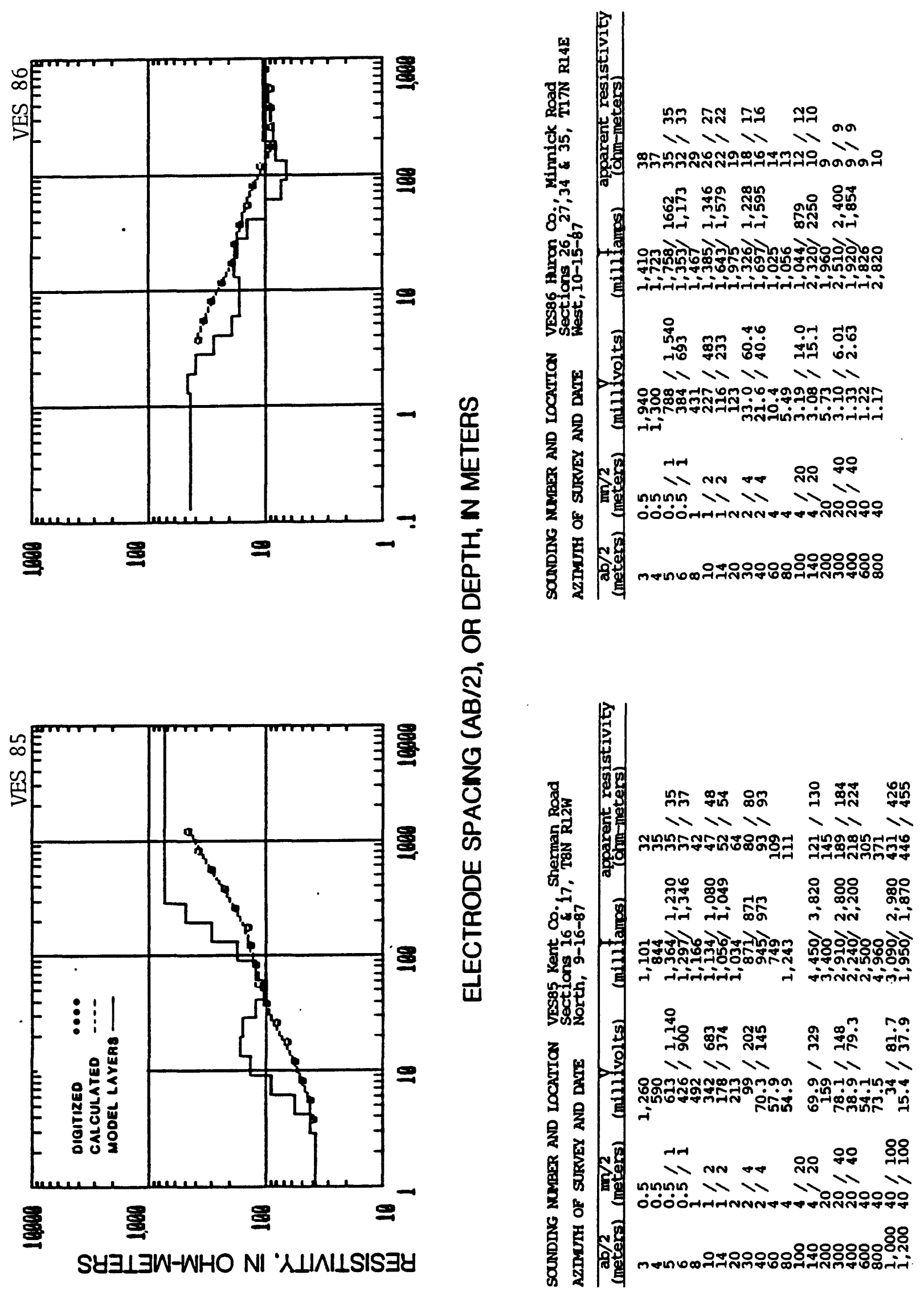

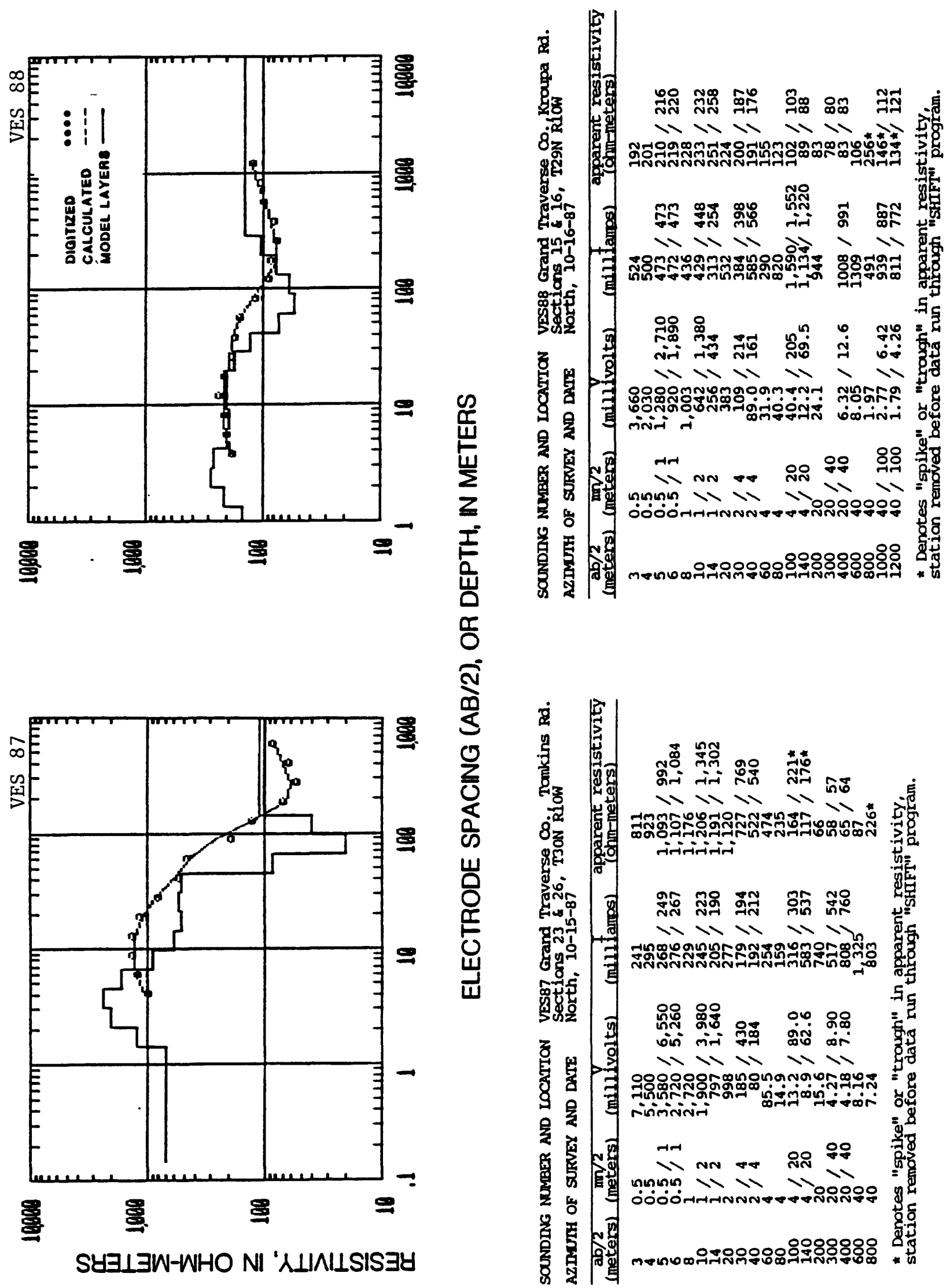

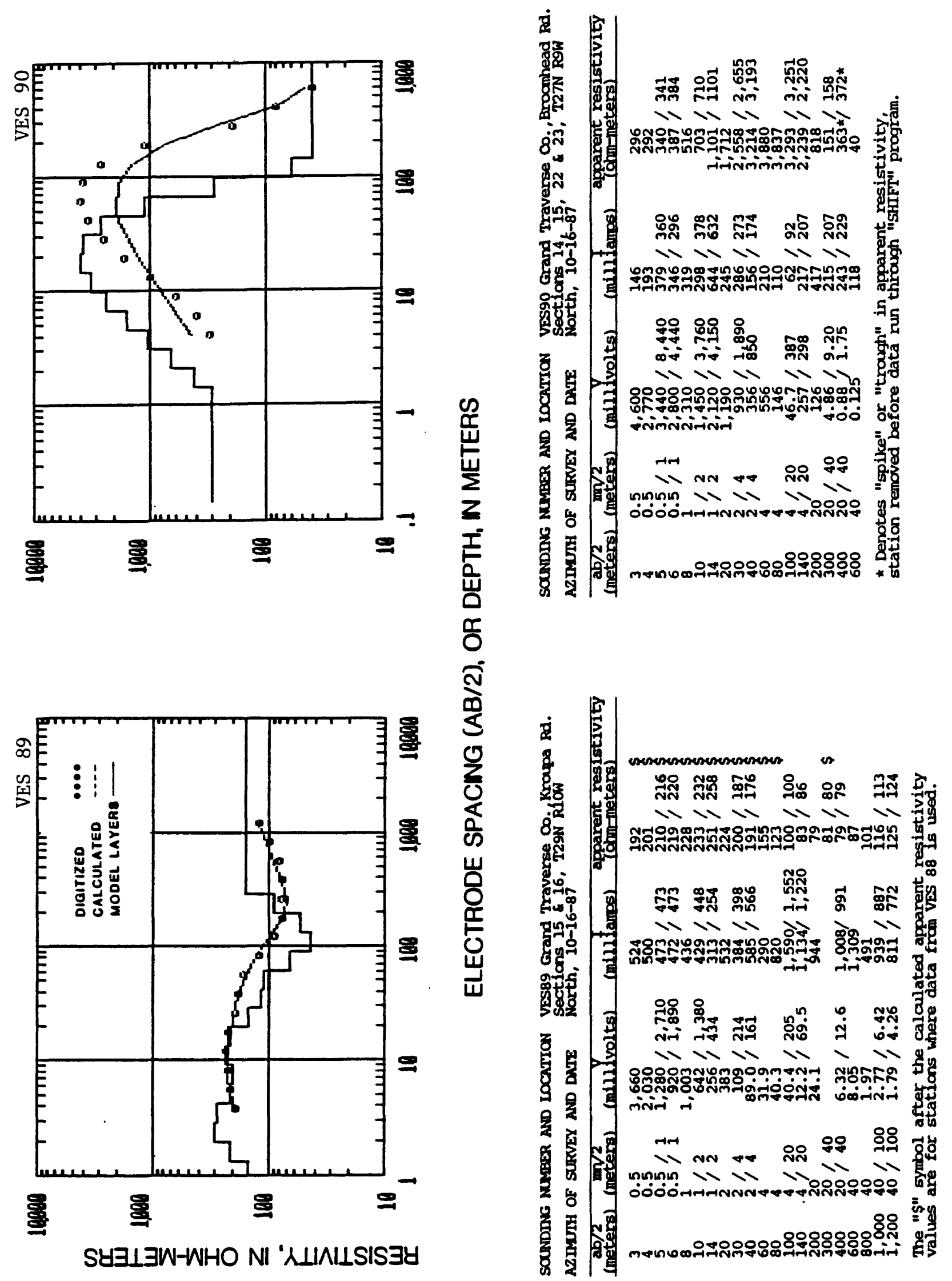


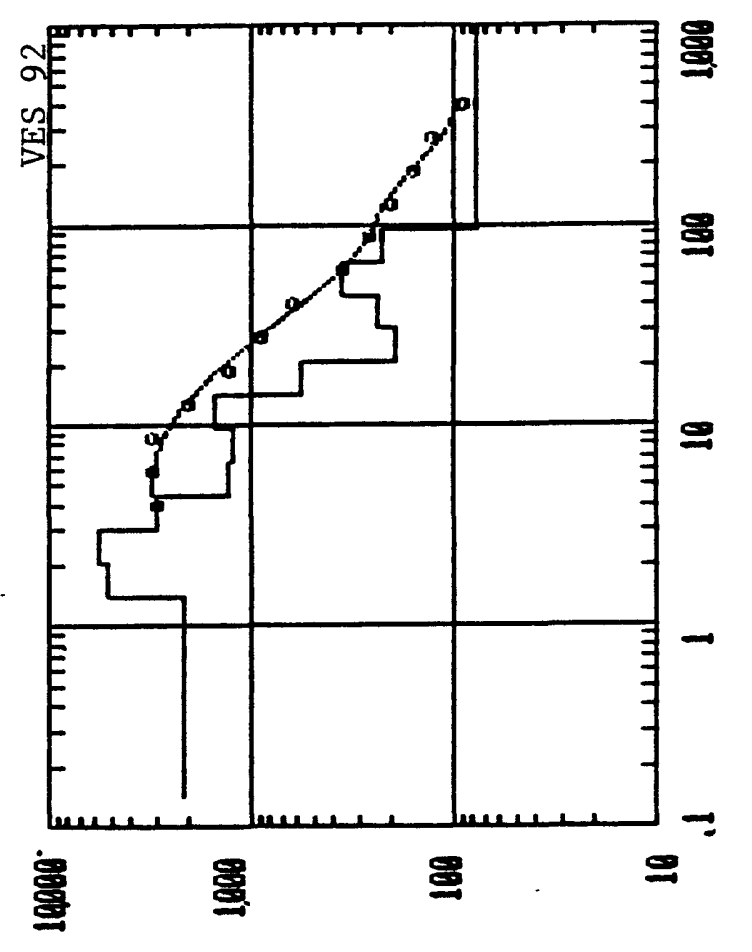

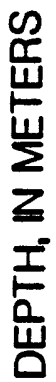

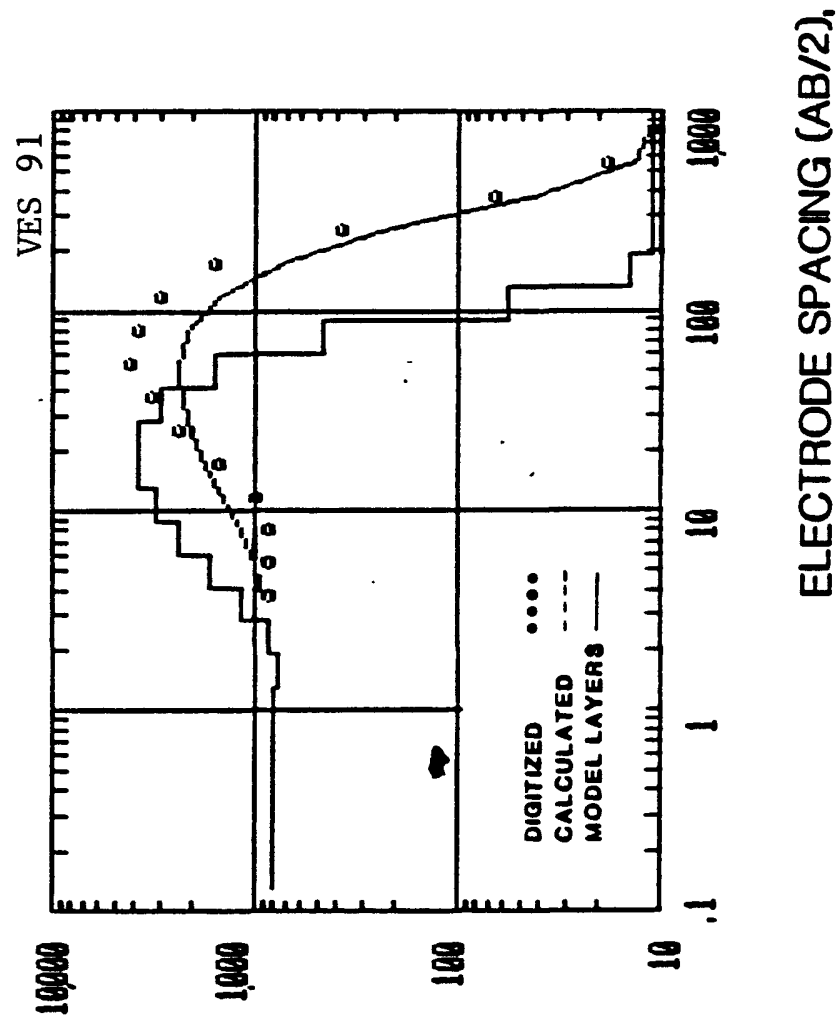

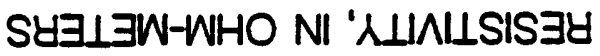
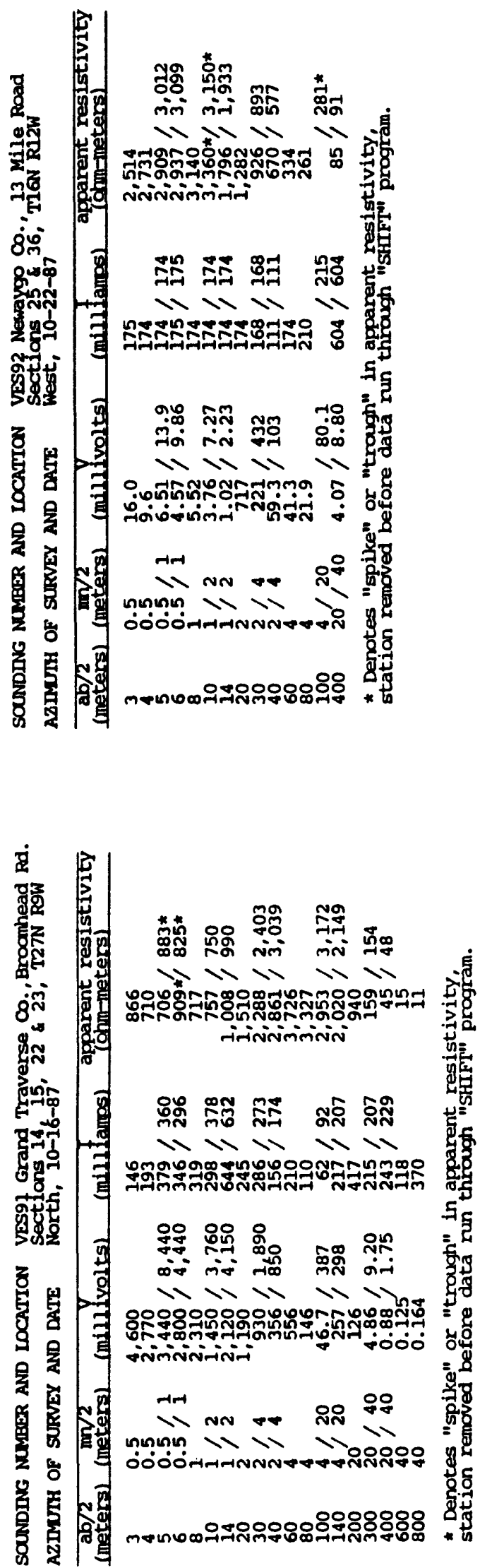


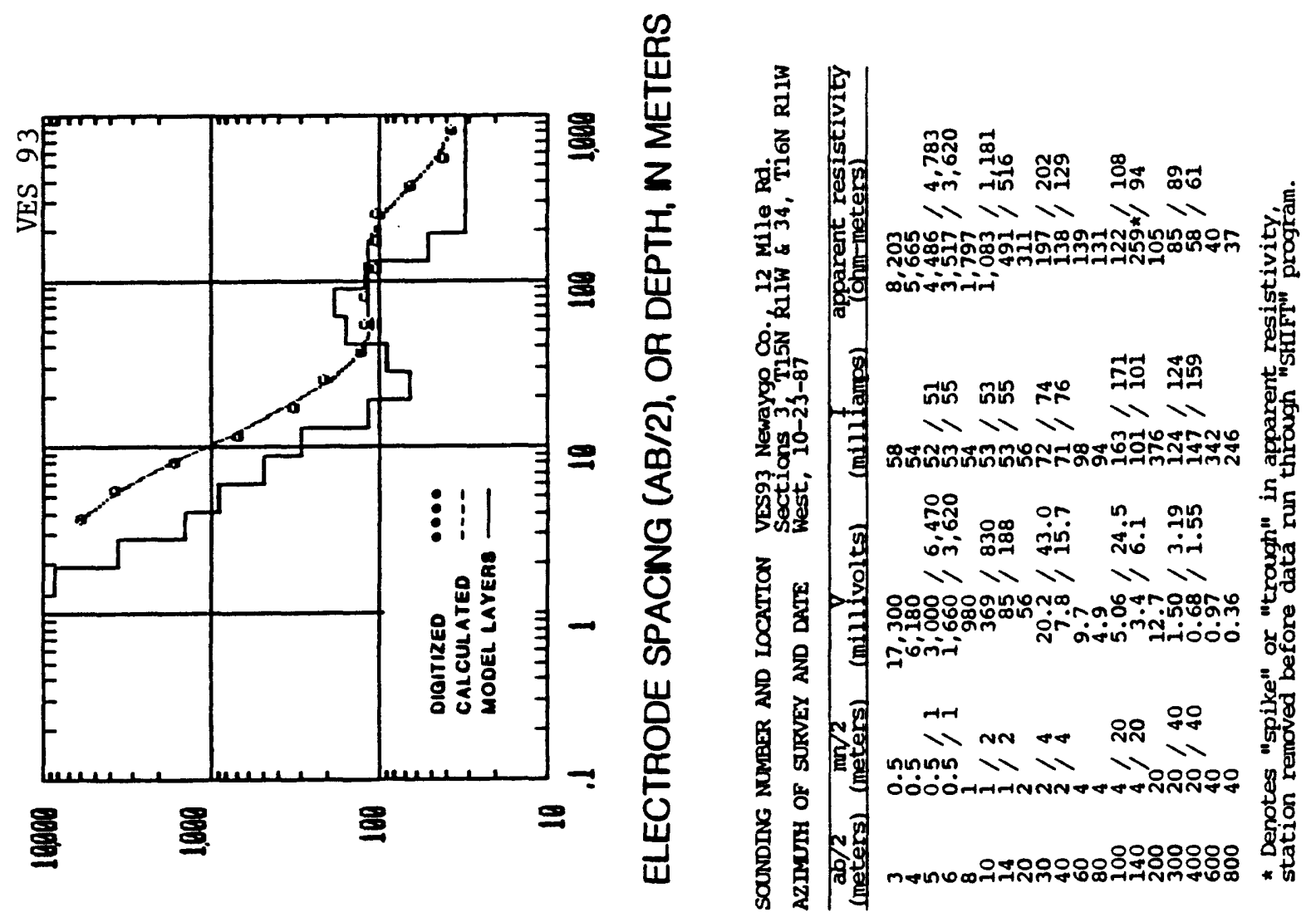

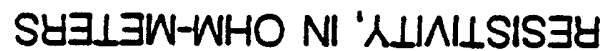

